NISTIR 8331

\title{
Ongoing Face Recognition Vendor Test (FRVT) Part 6B: Face recognition accuracy with face masks using post-COVID-19 algorithms
}

Mei Ngan

Patrick Grother

Kayee Hanaoka

This publication is available free of charge from:

https://doi.org/10.6028/NIST.IR.8331 
NISTIR 8331

\title{
Ongoing Face Recognition Vendor Test (FRVT) Part 6B: Face recognition accuracy with face masks using post-COVID-19 algorithms
}

\author{
Mei Ngan \\ Patrick Grother \\ Kayee Hanaoka \\ Information Access Division \\ Information Technology Laboratory
}

This publication is available free of charge from:

https://doi.org/10.6028/NIST.IR.8331

November 2020

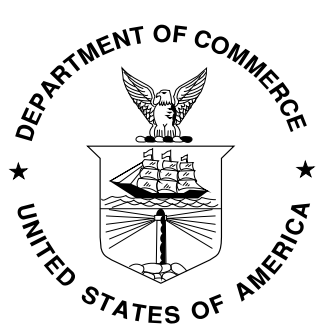

U.S. Department of Commerce

Wilbur L. Ross, Jr., Secretary

National Institute of Standards and Technology Walter Copan, NIST Director and Undersecretary of Commerce for Standards and Technology 
Certain commercial entities, equipment, or materials may be identified in this document in order to describe an experimental procedure or concept adequately. Such identification is not intended to imply recommendation or endorsement by the National Institute of Standards and Technology, nor is it intended to imply that the entities, materials, or equipment are necessarily the best available for the purpose.

National Institute of Standards and Technology Interagency or Internal Report 8331

Natl. Inst. Stand. Technol. Interag. Intern. Rep. 8331, 83 pages (November 2020)

This publication is available free of charge from: https://doi.org/10.6028/NIST.IR.8331 


\section{Executive Summary}

OVERVIEW

WHAT'S NEW

MOTIVATION

WHAT WE DID
This is the second of a series of reports on the performance of face recognition algorithms on faces occluded by protective face masks [2] commonly worn to reduce inhalation and exhalation of viruses. Inspired by the COVID-19 pandemic response, this is a continuous study being run under the Ongoing Face Recognition Vendor Test (FRVT) executed by the National Institute of Standards and Technology (NIST). In our first report [8], we tested "pre-pandemic" algorithms that were already submitted to FRVT 1:1 prior to mid-March 2020. This report augments its predecessor with results for more recent algorithms provided to NIST after mid-March 2020. While we do not have information on whether or not a particular algorithm was designed with face coverings in mind, the results show evidence that a number of developers have adapted their algorithms to support face recognition on subjects potentially wearing face masks. The algorithms tested were one-to-one algorithms submitted to the FRVT 1:1 Verification track. Future editions of this document will also report accuracy of one-to-many algorithms.

This report includes

$\triangleright$ Results from evaluating 65 face recognition algorithms provided to NIST since mid-March 2020

$\triangleright$ Assessment of when both the enrollment and verification images are masked (in addition to when only the verification image is masked)

$\triangleright$ Results for red and white colored masks (in addition to light-blue and black)

$\triangleright$ Cumulative results for 152 algorithms evaluated to date (submitted both prior to and after midMarch 2020)

Traditionally, face recognition systems (in cooperative settings) are presented with mostly nonoccluded faces, which include primary facial features such as the eyes, nose, and mouth. However, there are a number of circumstances in which faces are occluded by masks such as in pandemics, medical settings, excessive pollution, or laboratories. Inspired by the COVID-19 pandemic response, the widespread requirement that people wear protective face masks in public places has driven a need to understand how cooperative face recognition technology deals with occluded faces, often with just the periocular area and above visible. An increasing number of research publications have surfaced on the topic of face recognition on people wearing masks along with face-masked research datasets [10]. A number of commercial providers have announced the availability of face recognition algorithms capable of handling face masks, and this report documents performance results for 65 algorithms submitted to NIST after mid-March 2020. This report includes results for all algorithms evaluated to date. At the time of this writing, we are not aware of any large-scale, independent, and publicly reported evaluation on the effects of face mask occlusion on face recognition.

The NIST Information Technology Laboratory (ITL) quantified the accuracy of face recognition algorithms on faces occluded by masks applied digitally to a large set of photos that has been used in an FRVT verification benchmark since 2018. These algorithms were submitted to FRVT 1:1 and includes 65 new algorithms provided to NIST since mid-March 2020. While we do not have information on whether or not a particular algorithm was designed with face coverings in mind, the algorithms were submitted with the expectation that NIST would execute them on masked face images. Using the original unmasked images to form a baseline for accuracy, we measured the impact of occlusion by digitally applying a mask to the face and varying mask shape, mask color, and nose coverage.

We ran these algorithms over a large set of photographs collected in U.S. governmental applications that are currently in operation: application photographs from a global population of applicants for immigration benefits and border crossing photographs of travelers entering the United States. Both datasets were collected for authorized travel or immigration processes. 
WHAT WE DID (CONTINUED)

WHAT WE FOUND
The application photos (used as reference images) have good compliance with image capture standards. The border crossing photos (used as probe images) are not in good compliance with image capture standards given possible constraints on capture equipment, duration, facilities, and environment. We evaluated the case where the application photos were left unmasked, and synthetic masks were applied to the border crossing photos. This mimics an operational scenario where a person wearing a mask attempts to authenticate against a prior visa or passport photo. We also evaluated when both the application photos and border crossing photos were masked. This mimics, for example, a seamless travel scenario through an airport where a masked face image captured at check-in is enrolled and used during subsequent authentication attempts of the passenger still wearing a face mask. Together these datasets allowed us to process a total of 6.2 million images through a cumulative total of 152 algorithms.

Our use of software to apply masks to face images has the following advantages: it allows very rapid characterization of the effect of masks on face recognition; it allows controlled exploration of factors such as mask size, shape, and color; it affords repeatability, which is key to the fair comparison of algorithms; it scales to very large datasets - in our study, some 6.2 million photographs - which allows fine-grained characterization of false positive rates in addition to false negative rates. Conversely, our use of digital masks presents a number of limitations - please see the Limitations section of this executive summary for a more detailed discussion on the limitations of this study.

The following results represent observations on algorithms provided to NIST both before and after the COVID-19 pandemic to date. We do not have information on whether or not a particular algorithm was designed with face coverings in mind. The results documented capture a snapshot of algorithms submitted to the FRVT 1:1 in face recognition on subjects potentially wearing face masks.

$\triangleright$ False rejection performance: All algorithms submitted after the pandemic continue to give increased false non-match rates (FNMR) when the probes are masked. While a few pre-pandemic algorithms still remain within the most accurate on masked photos, some developers have submitted algorithms after the pandemic showing significantly improved accuracy and are now among the most accurate in our test. Using border crossing images, without masks, the most accurate algorithms will fail to authenticate about $0.3 \%$ of persons while falsely accepting no more than 1 in 100000 impostors (i.e. $F N M R=0.003$ at $F M R=0.00001$ ). With the highest coverage mask we tested and the most accurate algorithms, this failure rate rises to about $5 \%(\mathrm{FNMR}=0.05)$ among all algorithms. This is noteworthy given that around $70 \%$ of the face area is occluded by the mask. However, many algorithms submitted since mid-March 2020 remain much less tolerant: some algorithms that are quite competitive with unmasked faces (FNMR $<0.01)$ still fail to authenticate between $10 \%$ to $40 \%$ of masked images (FNMR $\rightarrow 0.4$ ).

See Figures 6, 7 and Table 5

For the case where both the enrollment and verification images are masked, interestingly, many algorithms show a reduction in false non-match rates compared to when only the verification image is masked, at a fixed threshold. While the reduction in FNMR is favorable, we observe much larger false match rates when both images are masked. These findings are discussed in subsequent sections of this executive summary.

See Figure 37

In cooperative access control applications, false rejections can traditionally be remedied by users making second attempts. This is effective when users correct pose, expression, or illumination aspects of their presentation. With masked faces, however, a second attempt may not be effective if the failure is a systematic property of the algorithm. 
WHAT WE

FOUND

(CONTINUED) $\triangleright$ Evolution of algorithms on face masks: We observe that a number of algorithms submitted since mid-March 2020 show notable reductions in error rates with face masks over their pre-pandemic predecessors. When comparing error rates for unmasked versus masked faces, the median FNMR across algorithms submitted since mid-March 2020 has been reduced by around $25 \%$ from the median pre-pandemic results. The figure below presents examples of developer evolution on both masked and unmasked datasets. For some developers, false rejection rates in their algorithms submitted since mid-March 2020 decreased by as much as a factor of 10 over their pre-pandemic algorithms, which is evidence that some providers are adapting their algorithms to handle face masks. However, in the best cases, when comparing results for unmasked images to masked images, false rejection rates have increased from $0.3 \%-0.5 \%$ (unmasked) to $2.4 \%-5 \%$ (masked). The current performance of face recognition with face masks is comparable to the state-of-the-art on unmasked images in mid-2017 [4].

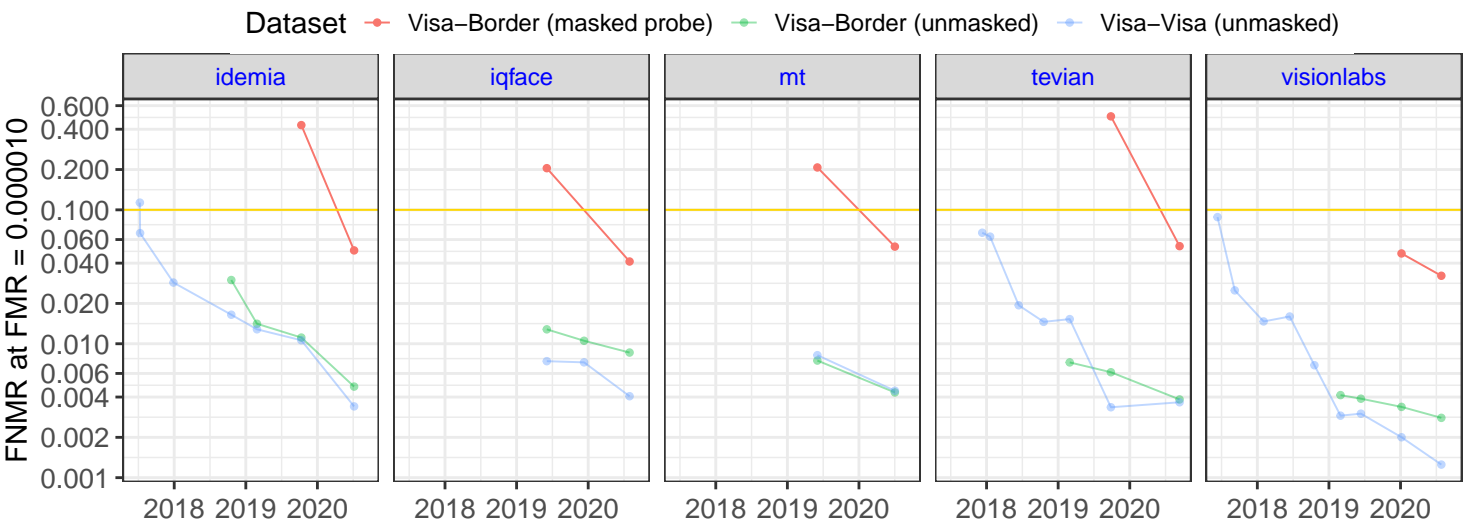

Figure 1: Examples of developer evolution of accuracy on masked and unmasked datasets.

See Figures 6, 5, and 7

$\triangleright$ False acceptance performance: As most systems are configured with a fixed threshold, it is necessary to report both false negative and false positive rates for each group at that threshold. When comparing a masked probe to an unmasked enrollment photo, in most cases, false match rates (FMR) are reduced by masks. The effect is generally modest with reductions in FMR usually being smaller than a factor of two. This property is valuable in that masked probes do not impart adverse false match security consequences for verification.

However, when both the enrollment and verification i mages a re m asked, m ost a lgorithms give elevated false match rates, with FMR ranging from 10 to 100 times higher than when only the probe is masked or both images are unmasked, at the same threshold. This behavior applies to most algorithms tested, with the exception of particular algorithms submitted by Idemia (idemia-006) and Pensees (pensees-001). These two algorithms generate approximately constant FMR across any masked/unmasked combination, and similarly, yield approximately constant FNMR across masked-probe and masked-enrollment-and-probe combinations.

See Figure 37

$\triangleright$ Mask-agnostic face recognition: All 1:1 verification algorithms submitted to the FRVT test since the start of the pandemic are evaluated on both masked and unmasked datasets. The test is designed this way to mimic operational reality: some images will have masks, some will not (especially enrollment samples from a database or ID card). And to the extent that the use of protective masks will exist for some time, our test will continue to evaluate algorithmic capability on verifying all combinations of masked and unmasked faces. 
WHAT WE

FOUND (CONTINUED)
At least two developers - Idemia and Pensees - have developed algorithms that work with any combination of masked and unmasked images, favorably generating approximately constant FMR across any masked/unmasked combination.
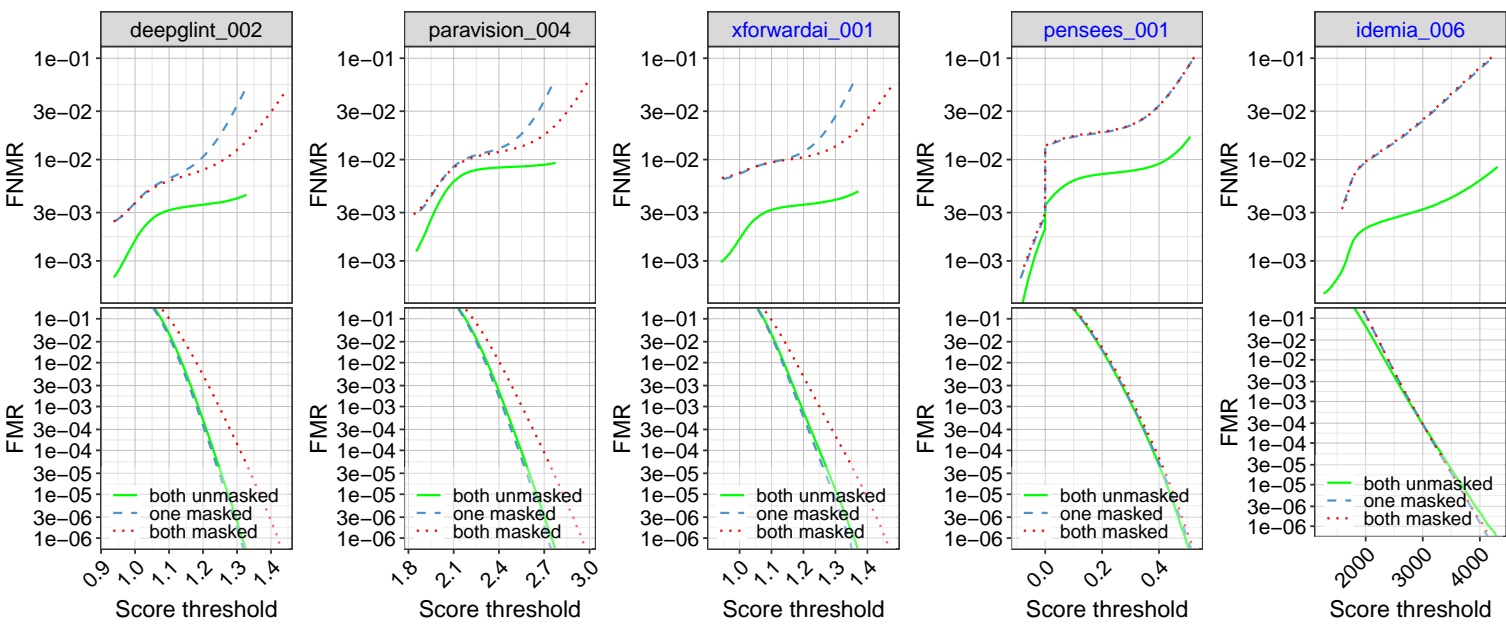

Figure 2: FNMR and FMR calibration curves on masked/unmasked combinations.

An example of an integrated approach might be: 1) inclusion of a mask detector 2) on an unmasked image, extract features from both the full face and the periocular region 3) on a detected masked image, extract features from the periocular region 4) at match time, compare full-face templates when both images are unmasked, and periocular templates otherwise.

$\triangleright$ Coverage of the masks: Masks that occlude more of the face give larger false non-match rates. We surveyed over the extent to which the mask covers the nose, from not at all ("low") to typical ("medium") to near the eyes ("high"). We baselined those with unmasked faces with the result that FNMR increases by factors of around 6,20, and 30 respectively for the median algorithm submitted since mid-March 2020. However, as noted, algorithms vary considerably in the their tolerance of coverage. Readers should consult tabulated values for specific algorithms.

See Table 5 and Figures 9, 11

We included the "low" option not because it is a common position for a mask but as an option for authentication applications where it would be tenable to ask the user to pull the mask down to just below the nose for the duration of the authentication attempt.

$\triangleright$ Color of the masks: We considered white, light-blue, red, and black masks. Many algorithms have higher error rates in black and red masks than light-blue and white masks. The reason for observed accuracy differences between mask color is unknown but is a point for consideration by impacted developers. Mask color also affects the rate at which some algorithms fail to produce a template from an image.

See Figure 24 and Table 9

$\triangleright$ Shape of the masks: The shape of the masks matters. Full-face-width masks generally cover more of the face than rounder N95 type masks. Post-pandemic algorithm results show that wide-width masks generally give false negative rates about a factor of 1.5 higher than do rounder type masks.

See Figure 8

$\triangleright$ Failure to detect and template: The false negative rates in this report include the effects of both face detection and localization errors, and low-similarity matching errors. We separately include tables detailing how often an algorithm does not make a template from an input image. While many algorithms give low failure-to-template rates, some give high values ranging close to $100 \%$. Conversely, the successful creation of a template does not guarantee proper facial localization. Such localization failures will not be captured as a failure to detect and template event but will impact accuracy rates nonetheless.

See Table 9 and Figure 10 
As a simulation, this study likely doesn't fully capture the effects of masks on face recognition. Particularly the following points should be weighed by readers in the near term. Some of these will be addressed in subsequent work at NIST.

$\triangleright$ Train algorithms: As with all NIST evaluations, we regard the software as a black box whose parameters (models) remain fixed for the entirety of its use without learning from the test data. We do not train or fine-tune algorithms.

$\triangleright$ Evaluate one-to-many algorithms: We have only run one-to-one verification algorithms with masks. This elicits data on the effect of masks on the underlying feature extraction and discrimination of algorithms and can therefore be be expected to give first-order indications of the effect on one-to-many identification algorithms.

$\triangleright$ Consider the effect of eye occlusion: We did not address the effect of eye-glasses or eye-protection. While our dataset includes examples of people wearing glasses, we didn't collect such data nor simulate it with digital addition.

$\triangleright$ Test with images of real masks: Given time and resource constraints, we didn't collect photos of subjects wearing masks. The possible downsides of this are several. First, our digital masks are tailored to faces; while a few don't fit realistically, mass-produced real masks may not fit all actual persons correctly either. We were not able to pursue an exhaustive simulation of the endless variations in color, design, shape, texture, bands, and ways masks can be worn. Second, because many cameras run with exposure-control, it is possible that a dark mask will cause less light to be reflecting and the camera to increase gain on the sensor causing overexposure of the periocular region. Likewise a white mask could lead to underexposure problems. Third, it is possible that some cameras that include a face detector, may fail to focus or acquire a masked face correctly.

$\triangleright$ Use textured masks: All masks synthesized by NIST in this study have a uniform color. The consequences of this are that we do not capture the impact of mask texture or pattern on face recognition. The possibility exists for patterned masks to induce higher facial localization errors, which is not captured in our current study. We received a suggestion that such information may serve as a soft biometric, in that a subject that always wears the same textured mask will be more identifiable. We don't intend to encourage algorithm development along this line, because as massproduced high-efficacy masks become more common, mask diversity may actually drop.

$\triangleright$ Study demographic effects on masked images: This report estimates overall performance of existing algorithms on recognition of faces occluded by masks. We deferred tabulating accuracy for different demographic groups until more capable mask-enabled algorithms have been submitted to FRVT.

$\triangleright$ Evaluate algorithms on non-cooperative, unconstrained imagery: This report documents results for matching masked webcam images to unmasked portrait-style photos. While the properties of the two sets of images differ, subjects are operating in cooperative mode and are for the most part, looking at the camera.

$\triangleright$ Consider effects of human examination: This report does not consider the various ways humans are involved in face recognition systems. For example, analysts can correct face detection or localization errors induced by masks, prior to automated recognition. Likewise, humans are often tasked with adjudication of images following a rejection or other exception from an automated system. Analysis of human capability and role is pertinent to those operations, but is beyond the scope of this study. 
IMPLICATIONS Know Your Algorithm: Operational implementations usually employ a single face recognition alAND FUTURE WORK gorithm. Given algorithm-specific sensitivities to masks and other image or subject properties, it is incumbent upon the system owner to know their algorithm. While publicly available test data from NIST and elsewhere can inform owners, it will usually be informative to specifically measure accuracy of the operational algorithm on the operational image data collected with actual masks.

NIST plans on releasing a series of reports, iteratively assessing different aspects and use cases of face masking on recognition performance. 


\section{ACKNOWLEDGMENTS}

This work was conducted in collaboration with the Department of Homeland Security's Science \& Technology Directorate (S\&T), Office of Biometric Identity Management (OBIM), and Customs and Border Protection (CBP). Additionally, the authors are grateful to staff in the NIST Biometrics Research Laboratory for infrastructure supporting rapid evaluation of algorithms.

\section{DISCLAIMER}

Specific hardware and software products identified in this report were used in order to perform the evaluations described in this document. In no case does identification of any commercial product, trade name, or vendor, imply recommendation or endorsement by the National Institute of Standards and Technology, nor does it imply that the products and equipment identified are necessarily the best available for the purpose.

\section{INSTITUTIONAL REVIEW BOARD}

The National Institute of Standards and Technology's Research Protections Office reviewed the protocol for this project and determined it is not human subjects research as defined in Department of Commerce Regulations, 15 CFR 27, also known as the Common Rule for the Protection of Human Subjects (45 CFR 46, Subpart A). 


\section{Contents}

EXECUTIVE SUMMARY I

ACKNOWLEDGMENTS

DISCLAIMER

INSTITUTIONAL REVIEW BOARD

1 FACE MASK EFFECTS 1

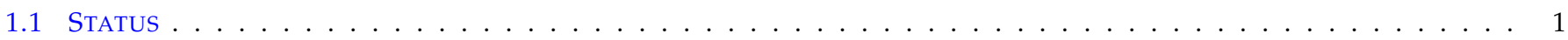

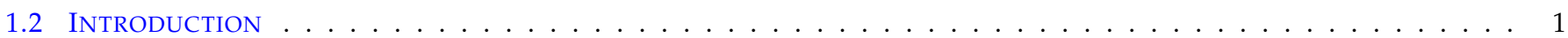

2 IMAGE DATASETS 2

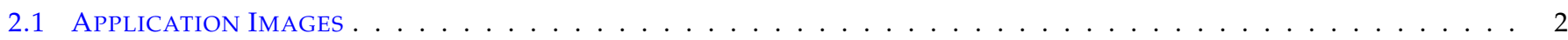

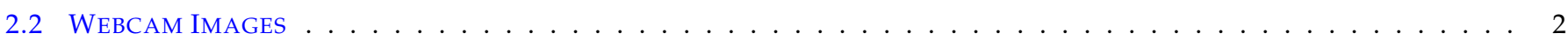

2.3 SYNTHETICALly MASKed IMAGES . . . . . . . . . . . . . . . . . . . . . . . . . 3

3 METRICS

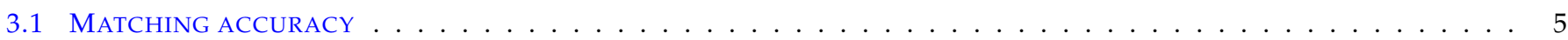

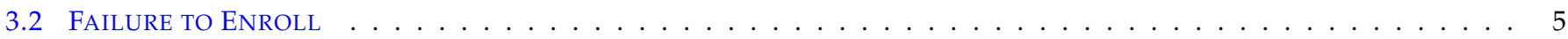

4 Algorithms 5

5 RESUlTS 9

EVOLUTION OF ALGORITHM PERFORMANCE WITH FACE MASKS . . . . . . . . . . . . . . . . . . . 9

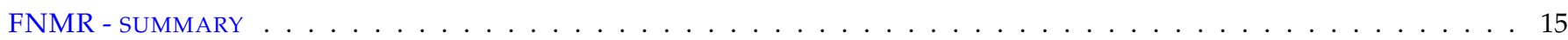

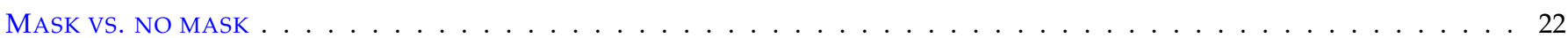

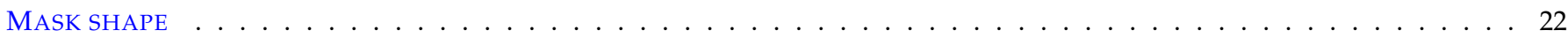

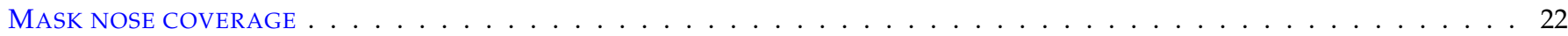

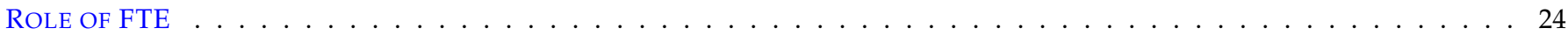

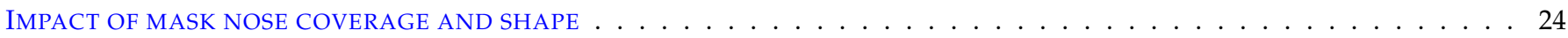

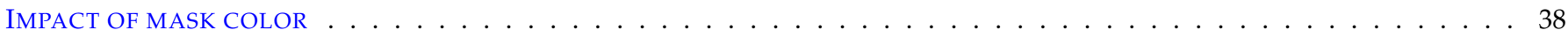

FNMR AND FMR VS. THRESHOLD . . . . . . . . . . . . . . . . . . . . . . . . . . . . . . . .

Appendix A Dlib Masking Methodology

\section{List of Tables}

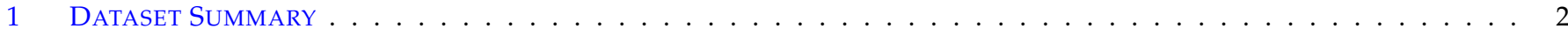

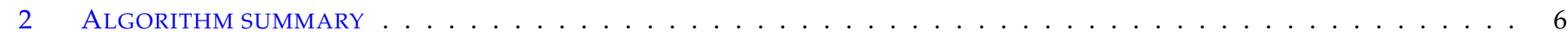

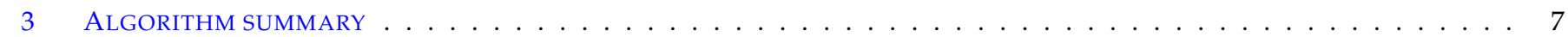

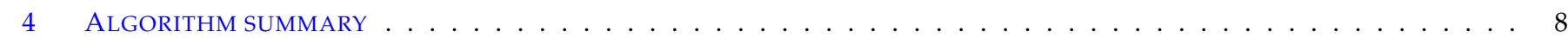

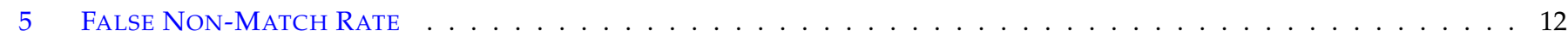

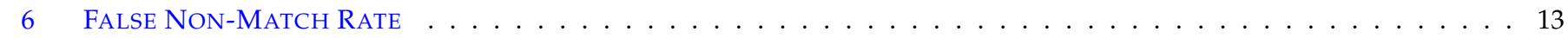

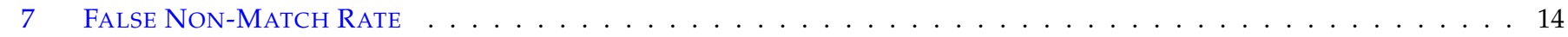

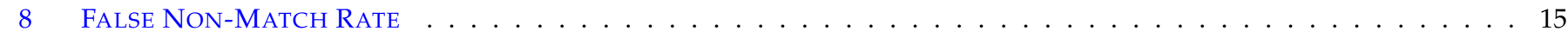

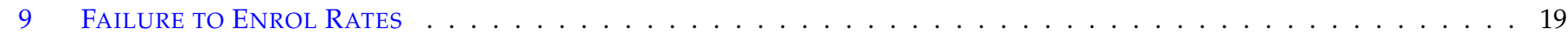

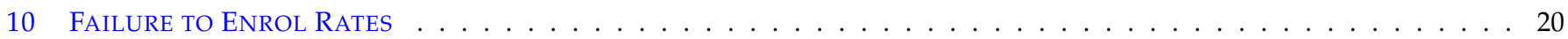

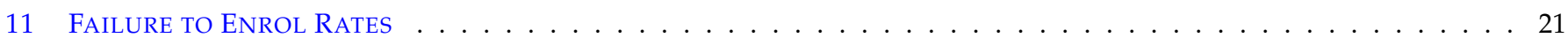

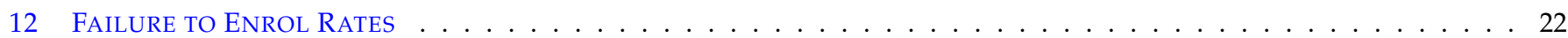




\section{List of Figures}

1 EXAMPLES OF DEVELOPER EVOLUtion OF ACCURACY ON MASKED AND UNMASKED DATASETS. . . . . . . . . . . . iii

2 FNMR AND FMR CALIBRATION CURVES ON MASKED/UNMASKED COMBINATIONS. . . . . . . . . . . . . . . . iv

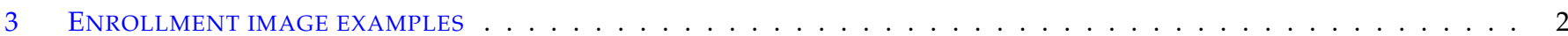

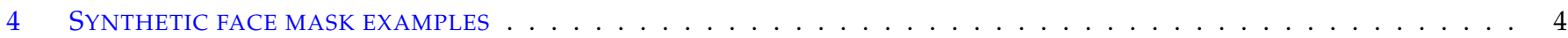

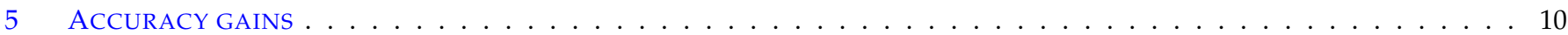

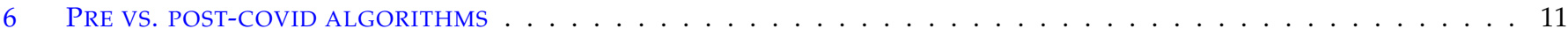

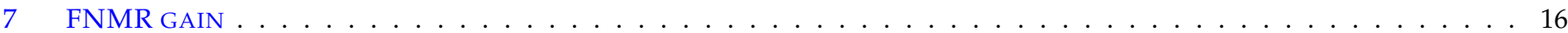

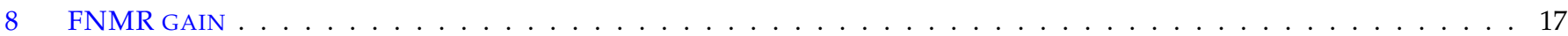

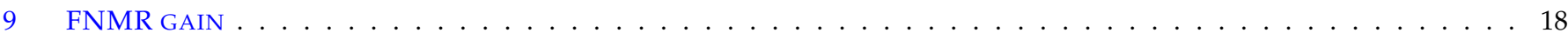

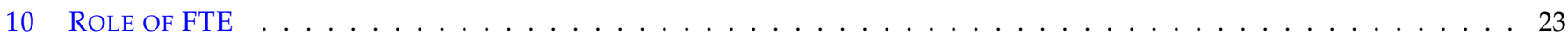

11 DET UNMASKED VerSUS MASKED - SHAPE AND COVERAGE . . . . . . . . . . . . . . . . . . . . . 25

12 DET UNMASKED VERSUS MASKED - SHAPE AND COVERAGE . . . . . . . . . . . . . . . . . . . . . 26

13 DET UNMASKED VERSUS MASKED - SHAPE AND COVERAGE . . . . . . . . . . . . . . . . . . . . . . 27

14 DET UNMASKED VERSUS MASKED - SHAPE AND COVERAGE . . . . . . . . . . . . . . . . . . . . 28

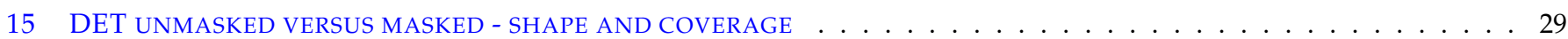

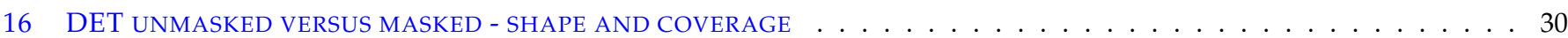

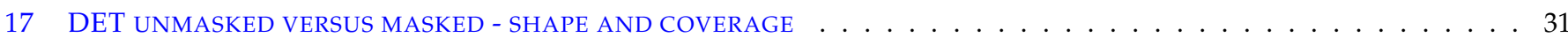

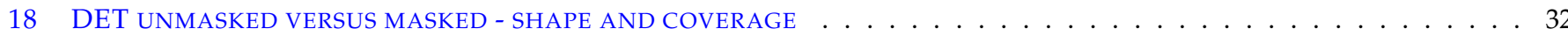

19 DET UNMASKED VERSUS MASKED - SHAPE AND COVERAGE . . . . . . . . . . . . . . . . . . . . . 33

20 DET UNMASKED VERSUS MASKED - SHAPE AND COVERAGE . . . . . . . . . . . . . . . . . . . . . 34

21 DET UNMASKED VERSUS MASKED - SHAPE AND COVERAGE . . . . . . . . . . . . . . . . . . . . . . 35

22 DET UNMASKED VERSUS MASKED - SHAPE AND COVERAGE . . . . . . . . . . . . . . . . . . . 36

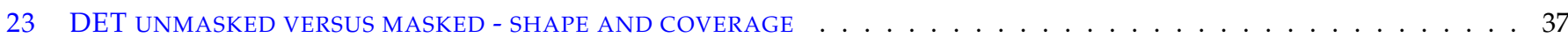

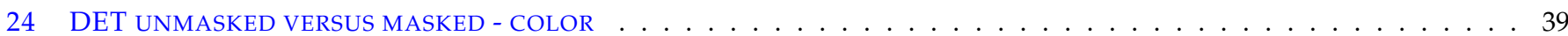

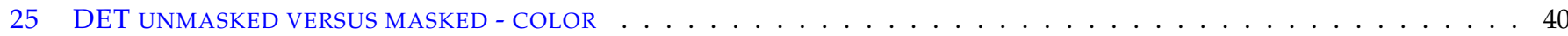

26 DET UNMASKED VERSUS MASKED - COLOR . . . . . . . . . . . . . . . . . . . . . . . . . . . 41

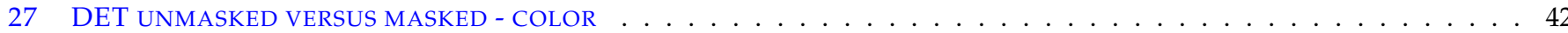

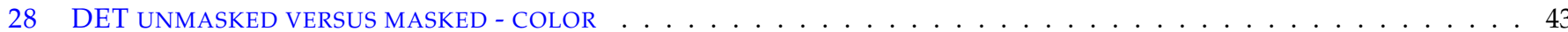

29 DET UNMASKED VERSUS MASKED - COLOR . . . . . . . . . . . . . . . . . . . . . . . . . . 44

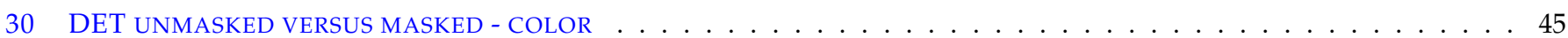

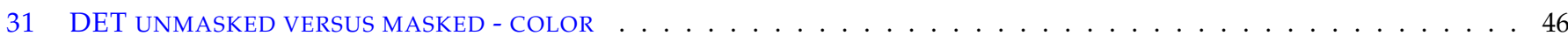

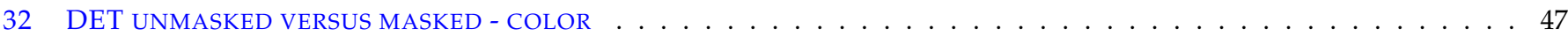

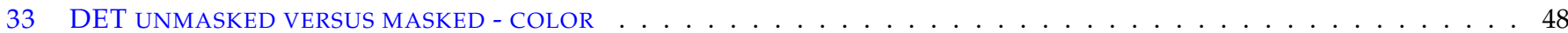

34 DET UNMASKED VERSUS MASKED - COLOR . . . . . . . . . . . . . . . . . . . . . . . . . 49

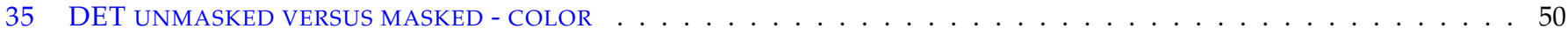

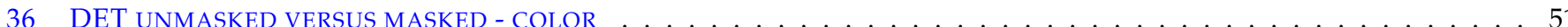

37 MASKED-TO-UNMASKED VS. MASKED-TO-MASKED

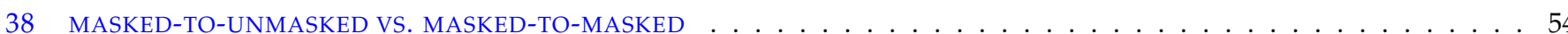

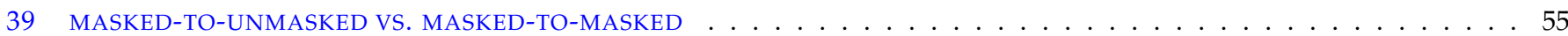

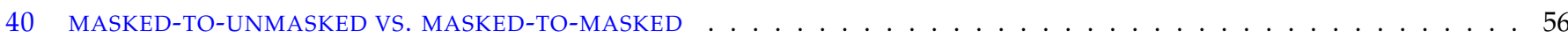

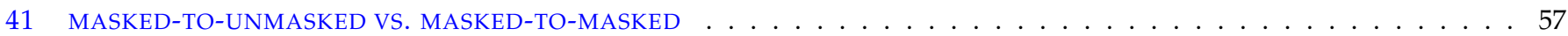

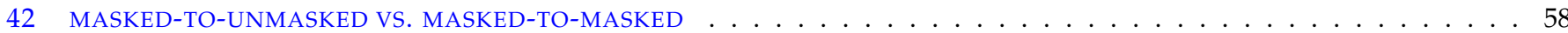

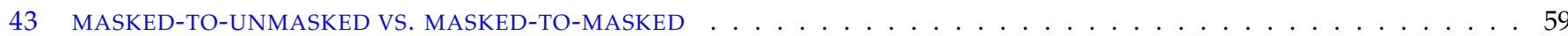

44 MASKED-TO-UNMASKED VS. MASKED-TO-MASKED

45 MASKED-TO-UNMASKED VS. MASKED-TO-MASKED

46 MASKED-TO-UNMASKED VS. MASKED-TO-MASKED

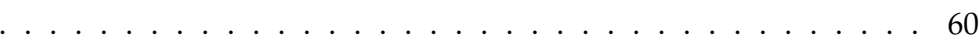

47 MASKED-TO-UNMASKED VS. MASKED-TO-MASKED

48 MASKED-TO-UNMASKED VS. MASKED-TO-MASKED

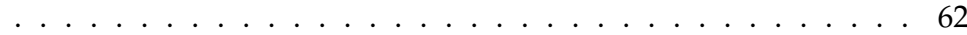

49 MASKED-TO-UNMASKED VS. MASKED-TO-MASKED

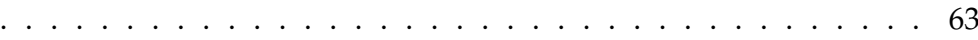

50 MASKED-TO-UNMASKED VS. MASKED-TO-MASKED

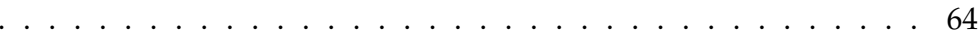

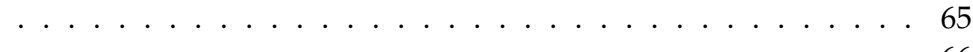

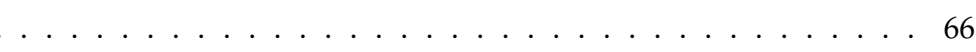

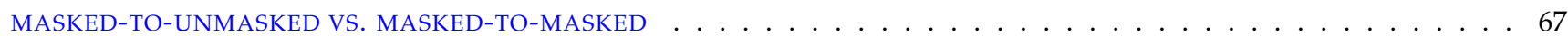

52 MASKED-TO-UNMASKED VS. MASKED-TO-MASKED

68

53 MASKED-TO-UNMASKED VS. MASKED-TO-MASKED

69

54 DLIB MASKING METHODOLOGY . 


\section{Face Mask Effects}

\subsection{Status}

NIST has conducted the second out of a series of tests aimed at quantifying face recognition accuracy for people wearing masks. Our initial approach has been to apply masks to faces digitally (i.e., using software to apply a synthetic mask). This allowed us to leverage large datasets that we already have. This report documents results for 1:1 verification algorithms. In our first report [8], we tested "pre-pandemic" algorithms that were already submitted to FRVT 1:1 prior to mid-March 2020. This report augments its predecessor with results for more recent algorithms provided to NIST after the COVID-19 pandemic. While we do not have information on whether or not a particular algorithm was designed with face coverings in mind, the algorithms were submitted with the expectation that NIST would execute them on masked face images. In addition to reporting results for when only the verification image is masked, we also document the effects for the case when both enrollment and verification images are masked. This report quantifies the effect of masks on both false negative and false positives match rates and tracks developer evolution of face recognition accuracy with face masks.

The FRVT evaluation is an ongoing test that remains open to new participation. Comments and suggestions should be directed to frvt@nist.gov.

\subsection{Introduction}

The majority of face recognition systems have been deployed in applications where subjects make cooperative presentations to a camera, for example as part of an application for a benefit or ID credential, or as during access control. With very few exceptions such images would not include face masks or other occlusions. However, with the COVID-19 pandemic, we can anticipate a demand to authenticate persons wearing masks, for example in immigration settings, without the need to the subjects to remove those masks. This presents a problem for face recognition, because regions of the face occluded by masks - the mouth and nose - include information useful for both recognition and, potentially, the detection stage that precedes it.

Previous work on face recognition of occluded faces has been directed at situations such as crime scenes where subjects were actively un-cooperative i.e. acting to evade face detection and recognition. Those applications are often characterized by image properties (low resolution, video compression, uncontrolled head orientation) that are known [5] to degrade recognition accuracy. 


\section{Image Datasets}

\subsection{Application Images}

The images are collected in an attended interview setting using dedicated capture equipment and lighting. The images are of size $300 \times 300$ pixels. The images are all high-quality frontal portraits collected in immigration offices and with a white background. As such, potential quality related drivers of high false match rates (such as blur) can be expected to be absent. The images are encoded as ISO/IEC 10918 i.e. JPEG. Over a random sample of 1000 images, the images have compressed file sizes (mean: $42 \mathrm{~KB}$, median: $58 \mathrm{~KB}, 25$-th percentile: $15 \mathrm{~KB}$, and 75 -th percentile: $66 \mathrm{~KB}$ ). The implied bitrates are mostly benign and superior to many e-Passports. When these images are provided as input into the algorithm, they are labeled with the type "ISO".
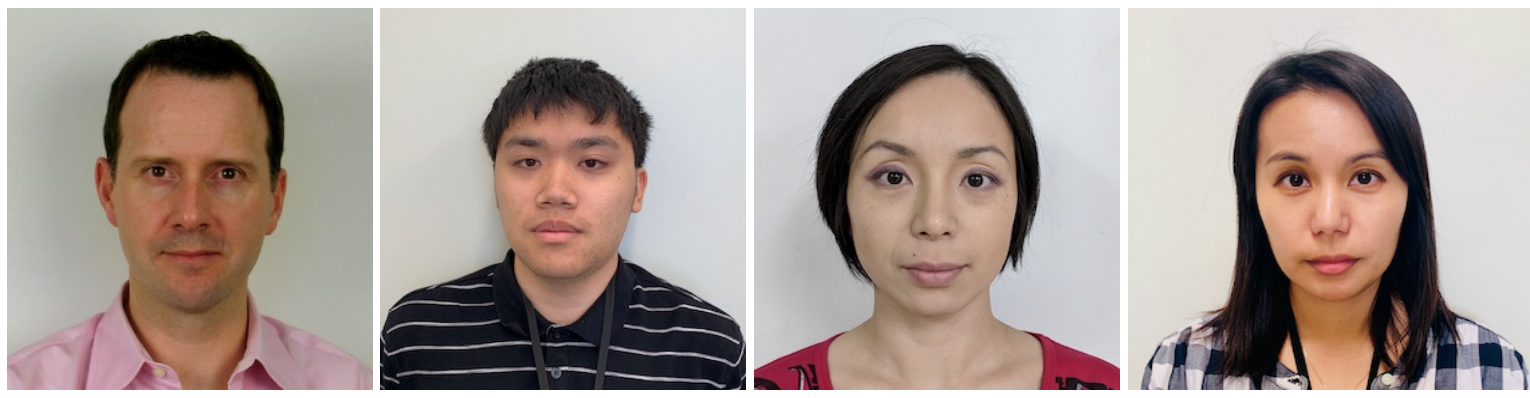

Figure 3: Examples of images with properties similar to the enrollment application photos used in this study. The subjects in the photos are all NIST employees.

\subsection{Webcam Images}

These images are taken with a camera oriented by an attendant toward a cooperating subject. This is done under time constraints, so there are roll, pitch, and yaw angle variations. Also, background illumination is sometimes bright, so the face is under exposed. Sometimes, there is perspective distortion due to close range images. The images are generally in poor conformance with the ISO/IEC 19794-5 Full Frontal image type. The images have mean interocular distance of 38 pixels. The images are all live capture. When these images are provided as input into the algorithm, they are labeled with the type "WILD". Examples of such images are included in Figure 4 and Figure 4 in NIST Interagency Report 8271. Results for verification of these images (unmasked) appear in FRVT Part 1 - Verification both compared against images of the same type, and with those described in section 2.1.

\begin{tabular}{|l|l|}
\hline Description & $\#$ \\
\hline \hline Total images & 6244865 \\
\hline Application (enrollment) images & 1019232 \\
\hline Subjects in application images & 1019232 \\
\hline Webcam (verification) images & 5225633 \\
\hline Subjects in webcam (verification) images & 2535329 \\
\hline Mated comparisons & 3225633 \\
\hline Impostor comparisons & 200000000 \\
\hline Subjects in mated comparisons & 535329 \\
\hline Subjects in impostor comparisons & 3019232 \\
\hline
\end{tabular}

Table 1: Summary quantities of the dataset used in this evaluation. 


\subsection{Synthetically Masked Images}

In this test, synthetically-generated masks were overlaid on top of 1) just the probe image (webcam images described in Section 2.2) or 2) both the enrollment (application photos described in Section 2.1) and probe images. The Dlib [7] C++ toolkit version 19.19 was used to detect and establish key facial points on the face, and with the facial points, solid masks of different shape, height, and color were drawn on the face. The exact Dlib facial points and details used to generate the masks are documented in Appendix A. In the event that Dlib was unable to detect a face in the image, eye coordinates were used to generate a mask leveraging standardized token frontal geometry [1].

Examples of synthetically-masked probe images are presented in Figures 4. 
ORIGINAL IMAGE
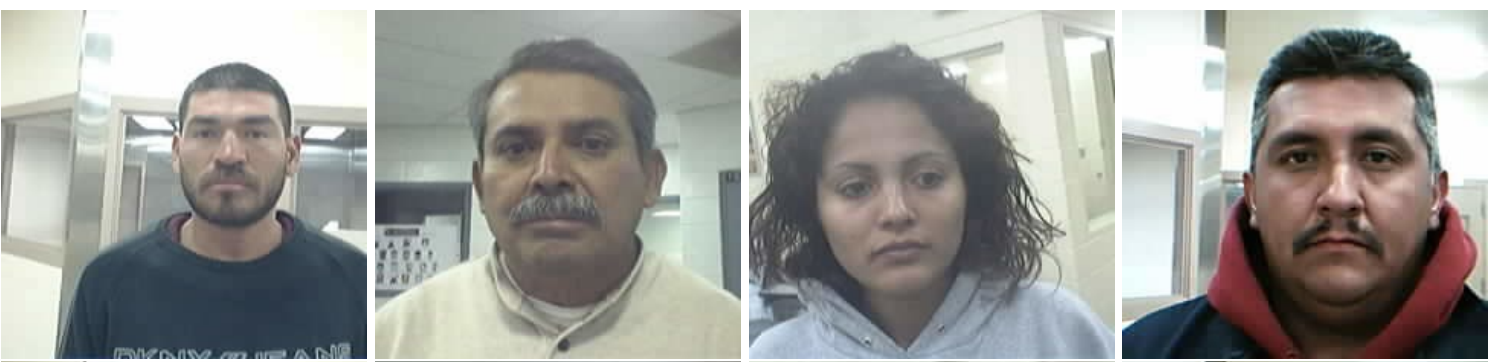

2

WIDE,

$\mathrm{HIGH}$

COVERAGE

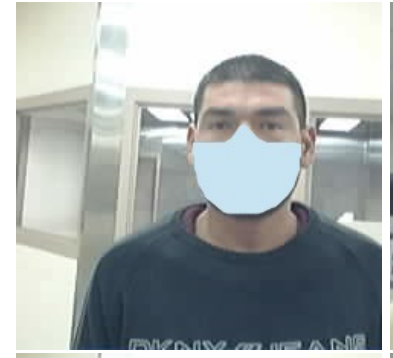

3

WIDE,

MEDIUM

COVERAGE

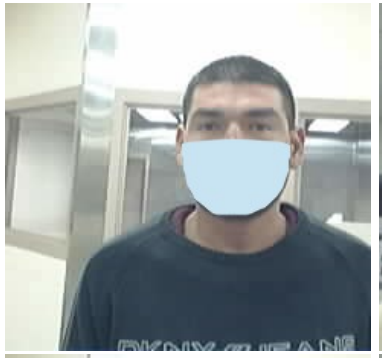

4

WIDE,

LOW

COVERAGE
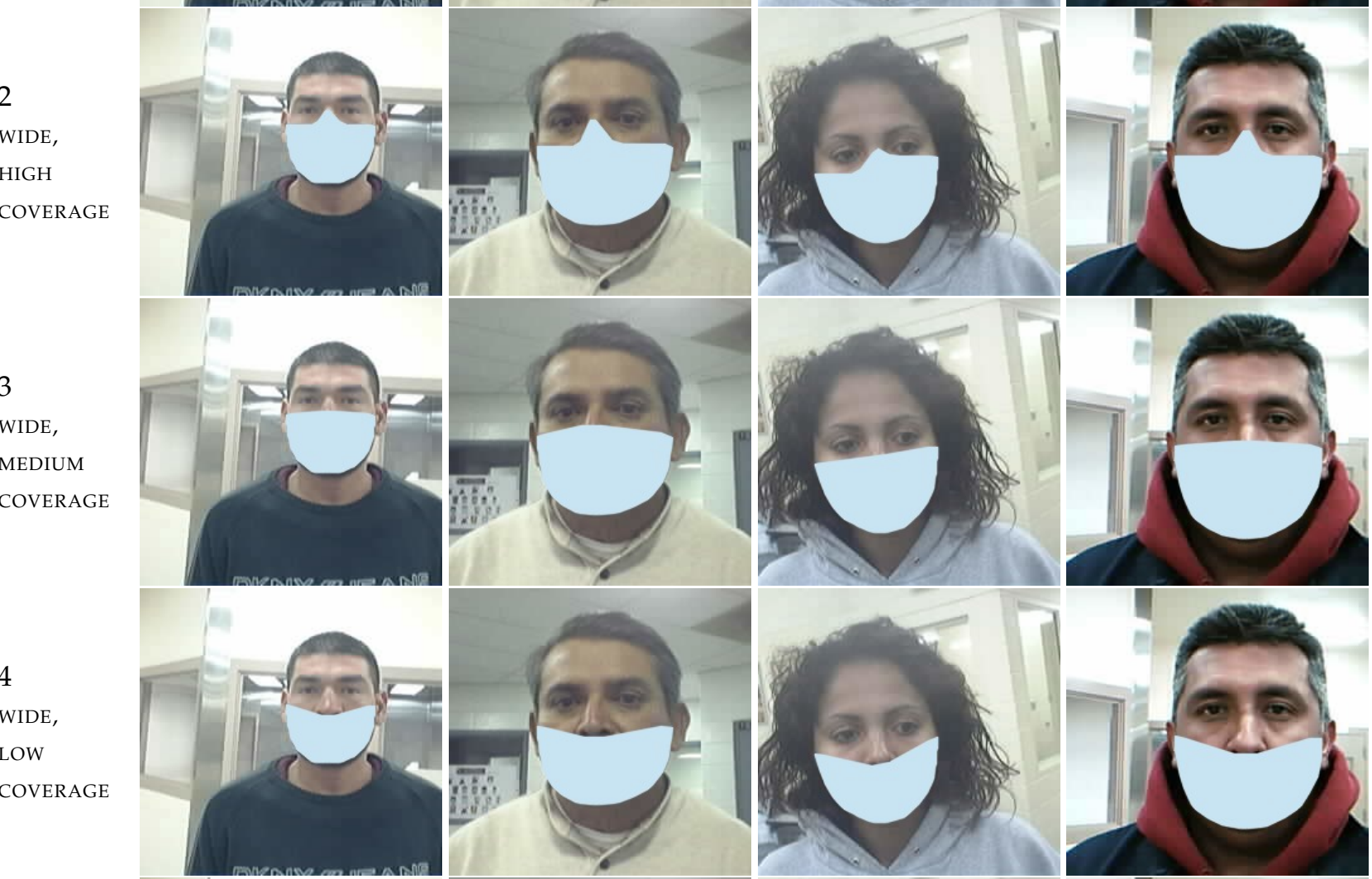

5

ROUND,

HIGH

COVERAGE
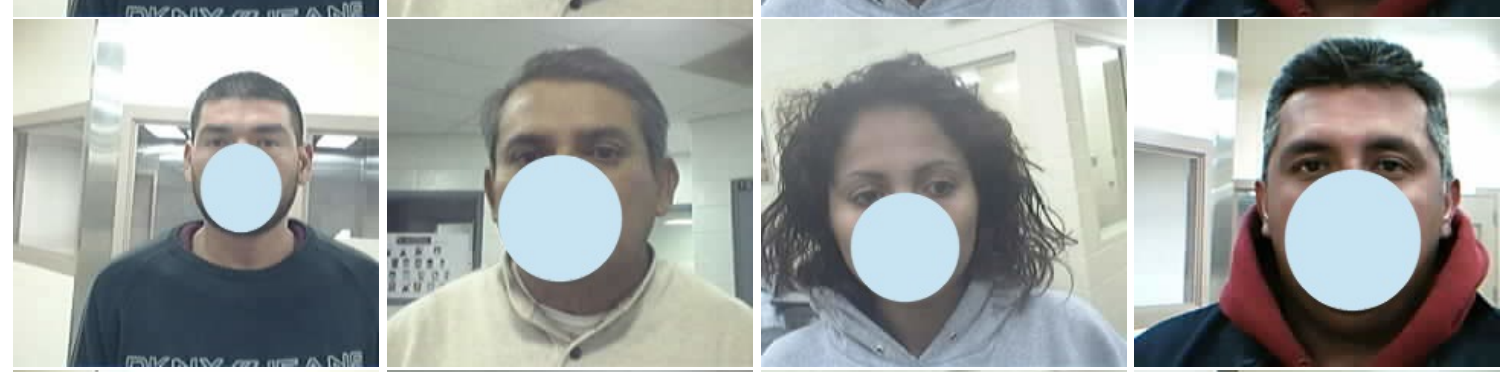

6

AS ROW 3

IN WHITE, LIGHT-BLUE, RED, AND BLACK
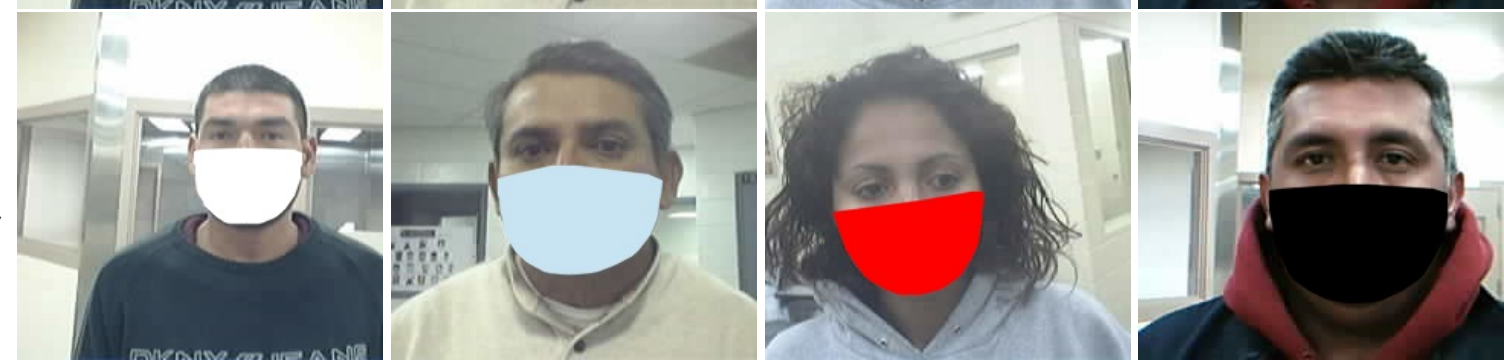

Figure 4: Examples of synthetically-generated face masks used in this study. The original images are from the NIST MEDS-II Dataset [3]. They were collected in operational settings using the same camera and procedure as is used for the border images that form the mainstay of the experiments in this report. 


\section{Metrics}

\subsection{Matching accuracy}

Given a vector of $\mathrm{N}$ genuine scores, $u$, the false non-match rate (FNMR) is computed as the proportion below some threshold, T:

$$
\operatorname{FNMR}(T)=1-\frac{1}{N} \sum_{i=1}^{N} H\left(u_{i}-T\right)
$$

where $H(x)$ is the unit step function, and $H(0)$ taken to be 1 .

Similarly, given a vector of $\mathrm{N}$ impostor scores, $v$, the false match rate (FMR) is computed as the proportion above T:

$$
\operatorname{FMR}(T)=\frac{1}{N} \sum_{i=1}^{N} H\left(v_{i}-T\right)
$$

The threshold, T, can take on any value. We typically generate a set of thresholds from quantiles of the observed impostor scores, $v$, as follows. Given some interesting false match rate range, $\left[\mathrm{FMR}_{L}, \mathrm{FMR}_{U}\right]$, we form a vector of $\mathrm{K}$ thresholds corresponding to FMR measurements evenly spaced on a logarithmic scale

$$
T_{k}=Q\left(1-\mathrm{FMR}_{k}\right)
$$

where $Q$ is the quantile function, and $\mathrm{FMR}_{k}$ comes from

$$
\log _{10} \mathrm{FMR}_{k}=\log _{10} \mathrm{FMR}_{L}+\frac{k}{K}\left[\log _{10} \mathrm{FMR}_{U}-\log _{10} \mathrm{FMR}_{L}\right]
$$

Detection error tradeoff (DET) characteristics are plots of FNMR(T) vs. FMR(T). These are plotted with FMR $\mathrm{FM}_{U} \rightarrow 1$ and $\mathrm{FMR}_{L}$ as low as is sustained by the number of impostor comparisons, N. This is somewhat higher than the "rule of three" limit $3 / N[6]$ because samples are not independent, due to re-use of images.

\subsection{Failure to Enroll}

Failure to enroll (FTE) is the proportion of failed template generation attempts. Failures can occur because the software throws an exception, or because the software electively refuses to process the input image. This would typically occur if a face is not detected. FTE is measured as the number of function calls that give EITHER a non-zero error code OR that give a "small" template. This is defined as one whose size is less than 60 bytes. This second rule is needed because some algorithms incorrectly fail to return a non-zero error code when template generation fails yet do return a valid default data structure.

The effects of FTE are included in the accuracy results of this report by regarding any template comparison involving a failed template to produce a low similarity score. Thus higher FTE results in higher FNMR and lower FMR.

\section{Algorithms}

The FRVT activity is open to participation worldwide, and the test will evaluate submissions on an ongoing basis. There is no charge to participate. The process and format of algorithm submissions to NIST are described in the FRVT 1:1 Verification Application Programming Interface (API) [9] document. Participants provide their submissions in the form of libraries compiled on a specific Linux kernel, which are linked against NIST's test harness to produce executables. NIST provides a validation package to participants to ensure that NIST's execution of submitted libraries produces the expected output on NIST's test machines. 
This report documents the results of algorithms submitted to FRVT 1:1 for testing both before (prior to mid-March 2020) and after the COVID-19 pandemic. Table 4 lists the algorithms that were tested. Note that algorithms that are expired or retired are not included in this report.

\begin{tabular}{|c|c|c|c|}
\hline & Developer & Algorithm & Submission Date \\
\hline 1 & 3Divi & 3divi-004 & $2019-07-22$ \\
\hline 2 & 3Divi & 3divi-005 & $2020-08-28$ \\
\hline 3 & ACI Software & acisw-003 & $2020-08-03$ \\
\hline 4 & ADVANCE.AI & advance-002 & 2019-12-19 \\
\hline 5 & ASUSTek Computer Inc & asusaics-000 & $2019-10-24$ \\
\hline 6 & AYF Technology & ayftech-001 & $2020-07-06$ \\
\hline 7 & Acer Incorporated & acer-000 & $2020-01-08$ \\
\hline 8 & Acer Incorporated & acer-001 & $2020-06-30$ \\
\hline 9 & Ai First & aifirst-001 & $2019-11-21$ \\
\hline 10 & AiUnion Technology & aiunionface- 000 & $2019-10-22$ \\
\hline 11 & Aigen & aigen-001 & $2020-10-06$ \\
\hline 12 & Akurat Satu Indonesia & ptakuratsatu-000 & $2020-09-11$ \\
\hline 13 & AlphaSSTG & alphaface-002 & $2020-02-20$ \\
\hline 14 & Anke Investments & anke-005 & 2019-11-21 \\
\hline 15 & Antheus Technologia & antheus-000 & 2019-12-05 \\
\hline 16 & Antheus Technologia & antheus-001 & $2020-06-25$ \\
\hline 17 & Aware & aware-005 & $2020-02-27$ \\
\hline 18 & Awidit Systems & awiros-001 & $2019-09-23$ \\
\hline 19 & Awidit Systems & awiros-002 & $2020-10-28$ \\
\hline 20 & Beihang University-ERCACAT & ercacat-001 & $2020-07-06$ \\
\hline 21 & Beijing Alleyes Technology & alleyes-000 & 2020-03-09 \\
\hline 22 & BioID Technologies SA & bioidtechswiss-000 & 2019-11-15 \\
\hline 23 & BioID Technologies SA & bioidtechswiss-001 & $2020-08-28$ \\
\hline 24 & Bresee Technology & bresee- 000 & $2020-08-07$ \\
\hline 25 & CSA IntelliCloud Technology & intellicloudai-001 & 2019-08-13 \\
\hline 26 & CTBC Bank & ctbcbank-000 & $2019-06-28$ \\
\hline 27 & Camvi Technologies & camvi-004 & $2019-07-12$ \\
\hline 28 & Canon Information Technology (Beijing) & cib-000 & $2019-12-11$ \\
\hline 29 & Canon Information Technology (Beiiing) & cib-001 & $2020-08-05$ \\
\hline 30 & China University of Petroleum & upc-001 & 2019-06-05 \\
\hline 31 & Chinese Univeristy of Hong Kong & cuhkee-001 & $2020-03-18$ \\
\hline 32 & Chosun University & chosun-000 & $2020-02-12$ \\
\hline 33 & Chosun University & chosun-001 & 2020-07-01 \\
\hline 34 & Chunghwa Telecom & chtface-002 & 2019-12-07 \\
\hline 35 & Chunghwa Telecom & chtface-003 & $2020-06-24$ \\
\hline 36 & Cortica & cor-001 & $2020-09-24$ \\
\hline 37 & Cybercore & cybercore-000 & $2020-08-26$ \\
\hline 38 & Cyberlink Corp & cyberlink-004 & $2020-02-27$ \\
\hline 39 & Cyberlink Corp & cyberlink-005 & $2020-07-31$ \\
\hline 40 & DSK & dsk-000 & $2019-06-28$ \\
\hline 41 & Dahua Technology & dahua-004 & $2019-12-18$ \\
\hline 42 & Dahua Technology & dahua-005 & $2020-08-13$ \\
\hline 43 & Decatur Industries Inc & decatur-000 & $2020-08-18$ \\
\hline 44 & Deepglint & deepglint-002 & 2019-11-15 \\
\hline 45 & DiDi ChuXing Technology & didiglobalface-001 & $2019-10-23$ \\
\hline 46 & Expasoft LLC & expasoft- 000 & $2020-01-06$ \\
\hline 47 & Expasoft LLC & expasoft-001 & $2020-09-03$ \\
\hline 48 & FaceSoft & facesoft-000 & $2019-07-10$ \\
\hline 49 & Fiberhome Telecommunication Technologies & fiberhome-nanjing-002 & $2020-08-10$ \\
\hline 50 & Fujitsu Research and Development Center & fujitsulab-000 & $2020-02-04$ \\
\hline 51 & Fujitsu Research and Development Center & fujitsulab-001 & $2020-09-30$ \\
\hline 52 & GeoVision Inc & geo-000 & $2020-06-29$ \\
\hline 53 & Glory & glory-002 & 2019-11-12 \\
\hline 54 & Gorilla Technology & gorilla-005 & $2020-03-11$ \\
\hline 55 & Gorilla Technology & gorilla-006 & $2020-07-31$ \\
\hline 56 & Guangzhou Pixel Solutions & pixelall-003 & $2019-10-15$ \\
\hline 57 & Guangzhou Pixel Solutions & pixelall-004 & $2020-07-02$ \\
\hline
\end{tabular}

Table 2: List of algorithms included in this report. Algorithms in black were submitted prior to mid-March 2020, and algorithms in blue were submitted thereafter. 


\begin{tabular}{|c|c|c|c|}
\hline & Developer & Algorithm & Submission Date \\
\hline 58 & Hengrui AI Technology & hr-003 & $2020-09-25$ \\
\hline 59 & ID3 Technology & id3-005 & $2020-08-04$ \\
\hline 60 & ITMO University & itmo-007 & 2020-01-06 \\
\hline 61 & Idemia & idemia-005 & $2019-10-11$ \\
\hline 62 & Idemia & idemia-006 & $2020-07-06$ \\
\hline 63 & Imageware Systems & iws-000 & $2020-08-12$ \\
\hline 64 & Imagus Technology Pty & imagus-001 & $2019-10-22$ \\
\hline 65 & Imperial College London & imperial-002 & $2019-08-28$ \\
\hline 66 & Incode Technologies Inc & incode-006 & $2020-02-20$ \\
\hline 67 & Incode Technologies Inc & incode- 007 & $2020-08-25$ \\
\hline 68 & Innef Labs & innefulabs-000 & 2020-09-04 \\
\hline 69 & Innovative Technology & innovativetechnologyltd-002 & $2020-02-26$ \\
\hline 70 & Innovatrics & innovatrics-006 & 2019-08-13 \\
\hline 71 & Institute of Information Technologies & iit-002 & 2019-12-04 \\
\hline 72 & Intel Research Group & intelresearch-001 & 2020-01-14 \\
\hline 73 & Intel Research Group & intelresearch-002 & $2020-07-24$ \\
\hline 74 & Intellivision & intellivision-002 & $2019-08-23$ \\
\hline 75 & Kakao Enterprise & kakao-003 & $2020-02-26$ \\
\hline 76 & Kedacom International Pte & kedacom-000 & 2019-06-03 \\
\hline 77 & Kneron Inc & kneron-005 & $2020-02-21$ \\
\hline 78 & Kookmin University & kookmin-001 & $2020-09-28$ \\
\hline 79 & Lomonosov Moscow State University & intsysmsu-002 & $2020-03-12$ \\
\hline 80 & Lookman Electroplast Industries & lookman-004 & 2019-06-03 \\
\hline 81 & Luxand Inc & luxand-000 & 2019-11-07 \\
\hline 82 & MVision & mvision-001 & 2019-11-12 \\
\hline 83 & Momentum Digital & sertis-000 & 2019-10-07 \\
\hline 84 & Momentum Digital & sertis-001 & $2020-07-30$ \\
\hline 85 & Moontime Smart Technology & $\mathrm{mt}-000$ & 2019-06-03 \\
\hline 86 & Moontime Smart Technology & $\mathrm{mt}-002$ & $2020-07-02$ \\
\hline 87 & N-Tech Lab & ntech-008 & 2020-01-06 \\
\hline 88 & NSENSE Corp & nsensecorp-001 & $2020-10-20$ \\
\hline 89 & Netbridge Technology Incoporation & netbridgetech-001 & $2020-01-08$ \\
\hline 90 & Netbridge Technology Incoporation & netbridgetech-002 & $2020-08-11$ \\
\hline 91 & Neurotechnology & neurotechnology-008 & 2020-01-08 \\
\hline 92 & Neurotechnology & neurotechnology-009 & $2020-07-07$ \\
\hline 93 & Nodeflux & nodeflux-002 & 2019-08-13 \\
\hline 94 & NotionTag Technologies Private Limited & notiontag-000 & 2019-06-12 \\
\hline 95 & Oz Forensics LLC & oz-001 & $2020-07-29$ \\
\hline 96 & PXL Vision AG & pxl-001 & $2020-06-30$ \\
\hline 97 & Panasonic R+D Center Singapore & psl-005 & $2020-07-06$ \\
\hline 98 & Paravision (EverAI) & paravision-004 & $2019-12-11$ \\
\hline 99 & Pensees Pte & pensees-001 & $2020-08-17$ \\
\hline 100 & Rank One Computing & rankone-009 & $2020-06-26$ \\
\hline 101 & Rank One Computing & rankone-010 & $2020-11-05$ \\
\hline 102 & Remark Holdings & remarkai-002 & $2019-11-21$ \\
\hline 103 & Rokid Corporation & rokid-000 & 2019-08-01 \\
\hline 104 & Samsung S1 Corp & s1-001 & 2019-12-06 \\
\hline 105 & Satellite Innovation/Eocortex & eocortex-000 & $2020-08-26$ \\
\hline 106 & Scanovate & scanovate-001 & $2019-11-12$ \\
\hline 107 & Scanovate & scanovate-002 & $2020-06-26$ \\
\hline 108 & Securif AI & securifai-001 & $2020-10-06$ \\
\hline 109 & Sensetime Group & sensetime-003 & 2019-06-04 \\
\hline 110 & Shanghai Jiao Tong University & sjtu-002 & $2020-02-12$ \\
\hline 111 & Shanghai Jiao Tong University & sjtu-003 & 2020-11-02 \\
\hline 112 & Shanghai Ulucu Electronics Technology & uluface-002 & 2019-07-10 \\
\hline 113 & Shanghai University - Shanghai Film Academy & shu-002 & $2019-12-10$ \\
\hline 114 & Shanghai University - Shanghai Film Academy & shu-003 & $2020-06-24$ \\
\hline
\end{tabular}

Table 3: List of algorithms included in this report. Algorithms in black were submitted prior to mid-March 2020, and algorithms in blue were submitted thereafter. 


\begin{tabular}{|c|c|c|c|}
\hline & Developer & Algorithm & Submission Date \\
\hline 115 & Shenzhen AiMall Tech & aimall-002 & $2020-03-12$ \\
\hline 116 & Shenzhen AiMall Tech & aimall-003 & $2020-08-12$ \\
\hline 117 & Shenzhen Intellifusion Technologies & intellifusion-002 & $2020-03-18$ \\
\hline 118 & Staqu Technologies & staqu-000 & $2020-07-15$ \\
\hline 119 & Star Hybrid Limited & starhybrid-001 & 2019-06-19 \\
\hline 120 & Su Zhou NaZhiTianDi intelligent technology & nazhiai-000 & $2020-06-25$ \\
\hline 121 & Synology Inc & synology-000 & $2019-10-23$ \\
\hline 122 & TUPU Technology & tuputech-000 & $2019-10-11$ \\
\hline 123 & Taiwan AI Labs & ailabs-001 & $2019-12-18$ \\
\hline 124 & Tech5 SA & tech5-004 & 2020-03-09 \\
\hline 125 & Tech5 SA & tech5-005 & $2020-07-24$ \\
\hline 126 & Tencent Deepsea Lab & deepsea-001 & 2019-06-03 \\
\hline 127 & Tevian & tevian-005 & $2019-09-21$ \\
\hline 128 & Tevian & tevian-006 & $2020-09-11$ \\
\hline 129 & Trueface.ai & trueface-000 & 2019-10-08 \\
\hline 130 & Trueface.ai & trueface-001 & $2020-07-20$ \\
\hline 131 & Universidade de Coimbra & visteam-000 & $2020-01-14$ \\
\hline 132 & Veridas Digital Authentication Solutions S.L. & veridas-004 & $2020-07-21$ \\
\hline 133 & Via Technologies Inc & via-001 & 2020-01-08 \\
\hline 134 & Videmo Intelligente Videoanalyse & videmo-000 & 2019-12-19 \\
\hline 135 & Videonetics Technology Pvt & videonetics-002 & 2019-11-21 \\
\hline 136 & Viettel Group & vts-000 & 2020-11-04 \\
\hline 137 & Vigilant Solutions & vigilantsolutions-007 & $2019-06-27$ \\
\hline 138 & Vigilant Solutions & vigilantsolutions-008 & $2020-08-03$ \\
\hline 139 & VinAI Research VietNam & vinai-000 & $2020-09-24$ \\
\hline 140 & VisionLabs & visionlabs-008 & 2020-01-06 \\
\hline 141 & VisionLabs & visionlabs-009 & $2020-07-27$ \\
\hline 142 & Vocord & vocord-008 & $2020-01-031$ \\
\hline 143 & Winsense & winsense-001 & $2019-10-16$ \\
\hline 144 & X-Laboratory & $\mathrm{x}$-laboratory-001 & 2020-01-21 \\
\hline 145 & Xforward AI Technology & xforwardai-000 & $2020-02-06$ \\
\hline 146 & Xforward AI Technology & xforwardai-001 & $2020-09-25$ \\
\hline 147 & Xiamen University & $\mathrm{xm}-000$ & $2020-10-19$ \\
\hline 148 & YooniK & yoonik-000 & $2020-06-24$ \\
\hline 149 & Yuan High-Tech Development & yuan-000 & $2020-06-30$ \\
\hline 150 & iQIYI Inc & iqface- 000 & 2019-06-04 \\
\hline 151 & iQIYI Inc & iqface-002 & $2020-07-30$ \\
\hline 152 & iSAP Solution Corporation & isap-002 & 2020-09-01 \\
\hline
\end{tabular}

Table 4: List of algorithms included in this report. Algorithms in black were submitted prior to mid-March 2020, and algorithms in blue were submitted thereafter. 


\section{Results}

This section includes accuracy results for the 152 one-to-one verification algorithms listed in Section 4, of which 65 were submitted to FRVT after mid-March 2020 and are labeled in blue in figures and tables throughout this report. We do not include speed and computational resource requirements - they are given in Table 1 in the FRVT 1:1 report. Missing entries generally mean the algorithm was not run on that particular mask variation due to time and resource constraints. The results, which span many pages, are comprised of:

$\triangleright$ Evolution of algorithm performance with face masks: Figure 5 shows the evolution of performance with face masks for developers that have submitted algorithms since mid-March 2020.

$\triangleright$ FNMR - summary: Figure 6 gives a summary of false non-matches rates between pre and post-COVID algorithms with a common type of mask. FNMR values are stated at a fixed threshold calibrated to give FMR $=0.00001$ on unmasked images.

$\triangleright$ FNMR - detailed: Table 5 tabulates false non-match rates by color, shape, and nose coverage. It includes also FNMR without any mask. FNMR values are stated at a fixed threshold calibrated to give FMR $=0.00001$ on unmasked images.

$\triangleright$ Mask vs. no mask: The scatter plot in Figure 7 shows variation across all algorithms of FNMR without masks against FNMR with a common type of mask, broken out by pre and post-COVID algorithms.

$\triangleright$ Mask shape: The scatter plot in Figure 8 shows for all algorithms the increase in false negative results for wide masks vs. narrower round masks, broken out by pre and post-COVID algorithms.

$\triangleright$ Mask nose coverage: The scatter plot in Figure 9 shows for all algorithms the increase in false negative rates for masks that substantially cover the nose and those pulled beneath the nose, broken out by pre and post-COVID algorithms.

$\triangleright$ FTE: Table 9 gives empirical failure-to-template results by color, shape, and nose coverage. The table was produced using 10000 images of each kind of mask.

$\triangleright$ FTE as contributor to FNMR: The FNMR results include failure-to-template rates (FTE). Figure 10 shows the proportion of template generation failures, broken out by pre and post-COVID algorithms.

$\triangleright$ DET - impact of mask nose coverage and shape: This section of figures shows detection error tradeoff characteristics for each algorithm, across different mask nose coverages and shapes.

$\triangleright$ DET - impact of mask color: This section of figures shows detection error tradeoff characteristics for each algorithm, across mask colors.

$\triangleright$ FNMR and FMR vs. threshold: This section of figures shows the explicit dependence of false non-match rate and false match rate on threshold. 
Dataset $\rightarrow$ Visa-Border (masked probe) $\rightarrow$ Visa-Border (unmasked) $\rightarrow$ Visa-Visa (unmasked)
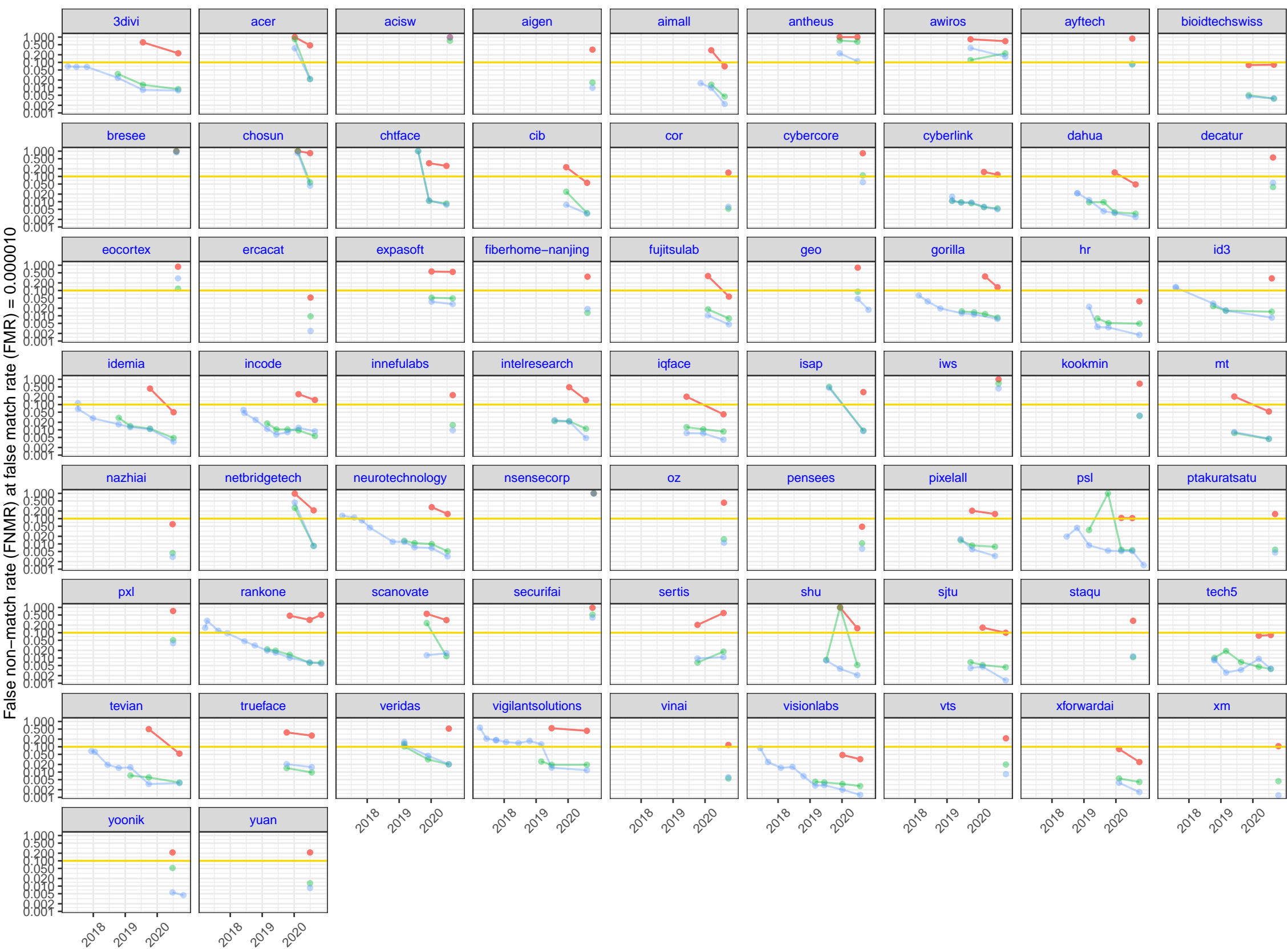

Date algorithm submitted to FRVT

Figure 5: Evolution of accuracy with face masks for developers that have submitted algorithms since mid-March 2020. Red line represents results on a masked dataset (masked probe), and for reference, the blue and green lines are results for unmasked datasets. FNMR on masked dataset is for medium, wide, lightblue masks. The horizontal gold line shows where FNMR=10\%. 
FNMR for medium wide lightblue masks

Algorithm Submission

Post-COVID

- Pre-COVID

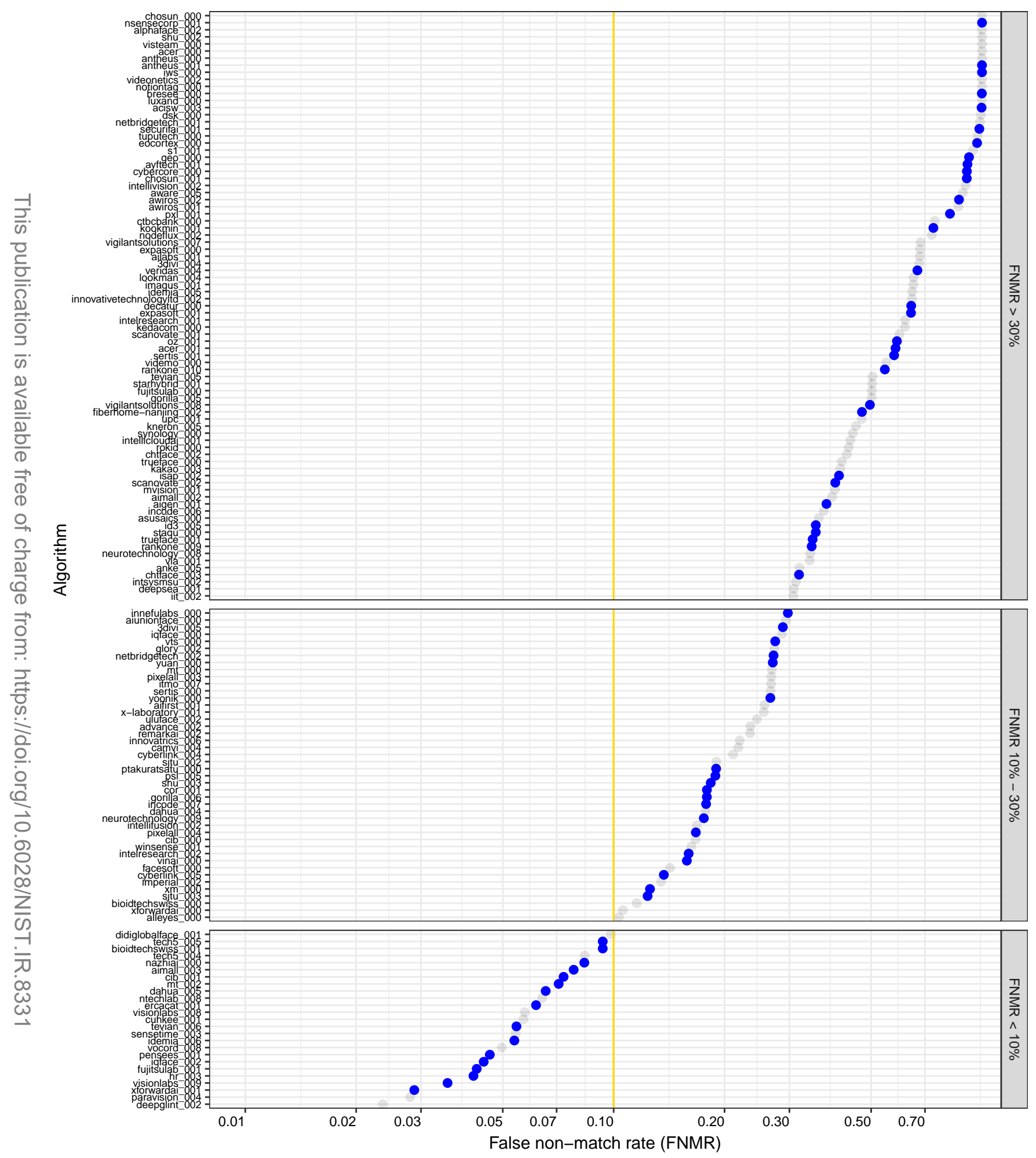

Figure 6: For each algorithm, each dot shows FNMR @ FMR=0.00001, where the threshold is set for FMR on unmasked probe images. The results are for when the probe is masked, and the enrollment image is unmasked. Gray dots represent results for algorithms submitted prior to mid-March 2020 (pre-COVID), and blue dots represent algorithms submitted thereafter (post-COVID). 


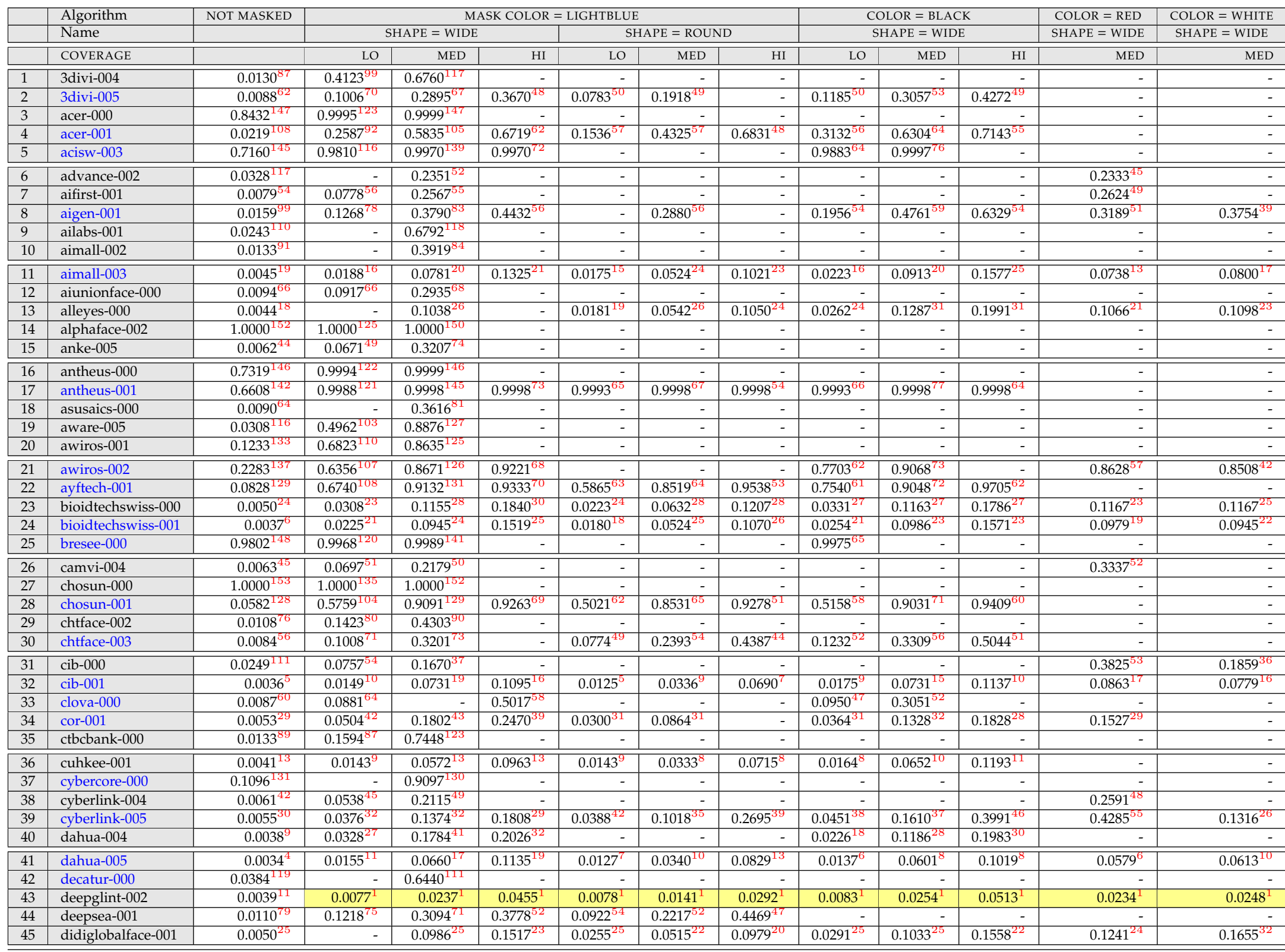

Table 5: This table summarizes False Non-Match Rate (FNMR) on unmasked and masked probe images. FNMR is the proportion of mated comparisons below a threshold set to achieve FMR=1e-05 on unmasked probe images. False Match Rate (FMR) is the proportion of impostor comparisons at or above that threshold. The red superscripts give rank over all algorithms in that column. Missing entries generally mean the algorithm was not run on that particular mask variation due to time and resource constraints. Algorithms with FTE=1.00 were not run at all. Algorithms in black were submitted prior to mid-March 2020, and algorithms in blue were submitted thereafter. 


\begin{tabular}{|c|c|c|c|c|c|c|c|c|c|c|c|c|c|}
\hline & \multirow{3}{*}{$\begin{array}{l}\text { Algorithm } \\
\text { Name } \\
\text { COVERAGE }\end{array}$} & \multirow{3}{*}{ NOT MASKED } & \multicolumn{6}{|c|}{ MASK COLOR = LIGHTBLUE } & \multirow{2}{*}{\multicolumn{3}{|c|}{$\begin{array}{l}\text { COLOR }=\text { BLACK } \\
\text { SHAPE }=\text { WIDE }\end{array}$}} & \multirow{3}{*}{$\begin{array}{r}\text { COLOR = RED } \\
\text { SHAPE = WIDE } \\
\text { MED }\end{array}$} & \multirow{3}{*}{$\begin{array}{r}\text { COLOR = WHITE } \\
\begin{array}{r}\text { SHAPE }=\text { WIDE } \\
\text { MED }\end{array}\end{array}$} \\
\hline & & & \multicolumn{3}{|c|}{ SHAPE $=$ WIDE } & \multicolumn{3}{|c|}{ SHAPE $=$ ROUND } & & & & & \\
\hline & & & LO & MED & $\mathrm{HI}$ & LO & MED & $\mathrm{HI}$ & LO & MED & $\mathrm{HI}$ & & \\
\hline$\overline{46}$ & $\begin{array}{c}\text { dsk-000 } \\
\end{array}$ & $0.1961^{134}$ & $0.9108^{113}$ & $0.9929^{138}$ & - & - & - & $\overline{-1}$ & - & - & $\overline{-1}$ & - & - \\
\hline 47 & eocortex-000 & $0.1187^{132}$ & - & $0.9694^{134}$ & - & - & - & - & - & - & - & - & - \\
\hline 48 & ercacat-001 & $0.0096^{69}$ & $0.0187^{15}$ & $0.0616^{15}$ & $0.0994^{14}$ & $0.0173^{14}$ & $0.0357^{11}$ & $0.0728^{10}$ & $0.0200^{13}$ & $0.0663^{12}$ & $0.1114^{9}$ & $0.0679^{12}$ & $0.0648^{12}$ \\
\hline 49 & expasoft- 000 & $0.0519^{\Gamma 26}$ & $0.3186^{96}$ & $0.6796^{119}$ & - & - & - & - & - & - & - & - & - \\
\hline 50 & expasoft-001 & $0.0492^{124}$ & - & $0.6414^{110}$ & - & - & - & - & - & - & - & - & - \\
\hline 51 & facesoft-000 & $0.0057^{36}$ & $0.0397^{34}$ & $0.1428^{33}$ & - & - & - & - & - & $0.1573^{36}$ & - & $0.1446^{28}$ & $0.1428^{29}$ \\
\hline 52 & fiberhome-nanjing-002 & $0.0133^{90}$ & $0.1554^{86}$ & $0.4734^{96}$ & - & - & - & - & $0.1641^{53}$ & - & - & - & - \\
\hline 53 & fujitsulab-000 & $0.0180^{103}$ & - & $0.5052^{101}$ & - & - & - & - & - & - & - & - & - \\
\hline 54 & fujitsulab-001 & $0.0078^{53}$ & $0.0330^{28}$ & $0.0427^{6}$ & $0.0608^{4}$ & $0.0311^{32}$ & $0.0363^{12}$ & $0.0666^{5}$ & $0.0407^{34}$ & $0.0985^{21}$ & $0.1525^{21}$ & $0.0898^{18}$ & $0.0776^{15}$ \\
\hline 55 & geo-000 & $0.0902^{130}$ & $0.5765^{105}$ & $0.9236^{132}$ & - & - & $0.8082^{63}$ & $0.9311^{52}$ & $0.6214^{60}$ & $0.9428^{74}$ & $0.9541^{61}$ & - & \\
\hline$\overline{56}$ & glory-002 & $0.0109^{78}$ & - & $0.2729^{64}$ & - & $\overline{-}$ & - & - & - & - & - & - & - \\
\hline 57 & gorilla-005 & $0.0117^{83}$ & $0.1463^{82}$ & $0.5037^{99}$ & - & - & & - & - & - & - & - & - \\
\hline 58 & gorilla-006 & $0.0085^{57}$ & $0.0495^{41}$ & $0.1805^{44}$ & $0.2883^{43}$ & $0.0385^{41}$ & $0.1134^{42}$ & - & 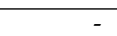 & $0.2260^{46}$ & $0.3419^{43}$ & $0.1980^{40}$ & \\
\hline 59 & hr-003 & $0.0049^{23}$ & $0.0133^{5}$ & $0.0419^{5}$ & $0.0613^{5}$ & $0.0122^{3}$ & $0.0247^{4}$ & $0.0475^{4}$ & $0.0142^{7}$ & $0.0527^{6}$ & $0.0914^{5}$ & $0.0542^{5}$ & $0.0476^{7}$ \\
\hline 60 & id3-005 & $0.0145^{96}$ & $0.1181^{74}$ & $0.3540^{80}$ & - & - & - & - & - & - & - & - & - \\
\hline$\overline{61}$ & idemia-005 & $0.0111^{81}$ & $0.2051^{90}$ & $0.6469^{113}$ & $0.6968^{64}$ & $0.1349^{56}$ & $0.4387^{58}$ & $\overline{-}$ & $0.2786^{55}$ & $0.7402^{69}$ & $0.8119^{56}$ & $\overline{-}$ & \\
\hline 62 & idemia-006 & $0.0048^{22}$ & $0.0327^{26}$ & $0.0539^{10}$ & $0.0911^{10}$ & $0.0322^{33}$ & $0.0440^{19}$ & $0.0946^{17}$ & $0.0359^{29}$ & $0.0714^{14}$ & $0.1489^{19}$ & $0.0607^{8}$ & $0.0564^{9}$ \\
\hline 63 & iit-002 & $0.0141^{94}$ & & $0.3078^{70}$ & - & - & - & - & - & - & - & - & - \\
\hline 64 & imagus-001 & $0.0276^{113}$ & $0.3488^{98}$ & $0.6510^{114}$ & - & - & - & - & - & - & - & - & - \\
\hline 65 & imperial-002 & $0.0055^{31}$ & $0.0320^{25}$ & $0.1350^{31}$ & $0.1972^{31}$ & $0.0258^{26}$ & $0.0775^{30}$ & $0.1556^{29}$ & $0.0359^{30}$ & $0.1510^{35}$ & $0.2302^{36}$ & $0.1533^{30}$ & $0.1432^{30}$ \\
\hline 66 & incode-006 & $0.0095^{68}$ & - & $0.3725^{82}$ & - & - & - & - & - & - & - & - & - \\
\hline 67 & incode- 007 & $0.0058^{37}$ & $0.0470^{38}$ & $0.1790^{42}$ & $0.2393^{38}$ & $0.0323^{34}$ & $0.1040^{37}$ & $0.1779^{31}$ & $0.0561^{41}$ & $0.1891^{43}$ & $0.2754^{39}$ & $0.1978^{39}$ & -5 \\
\hline 68 & innefulabs- 000 & $0.0155^{98}$ & - & $0.2971^{69}$ & - & - & - & - & - & - & - & - & - \\
\hline 69 & innovativetechnologyltd-002 & $0.0251^{112}$ & $0.2701^{93}$ & $0.6454^{112}$ & - & & & - & - & - & - & - & - \\
\hline 70 & innovatrics-006 & $0.0059^{40}$ & $0.0543^{48}$ & $0.2210^{51}$ & $0.3118^{44}$ & $0.0369^{37}$ & $0.1109^{40}$ & $0.1984^{33}$ & $0.0557^{40}$ & $0.1909^{44}$ & $0.2764^{40}$ & $0.1936^{38}$ & ${ }_{-}$ \\
\hline 71 & intellicloudai-001 & $0.0095^{67}$ & $0.1044^{72}$ & $0.4394^{92}$ & $\overline{-1-}$ & - & - & - & - & - & - & - & - \\
\hline 72 & intellifusion-002 & $0.0056^{35}$ & $0.0539^{46}$ & $0.1690^{39}$ & - & - & - & - & - & $0.1822^{42}$ & - & $0.2556^{47}$ & $0.2119^{37}$ \\
\hline 73 & intellivision-002 & $0.0463^{123}$ & $0.5999^{106}$ & $0.9028^{128}$ & - & - & - & - & - & - & - & - & 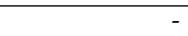 \\
\hline 74 & intelresearch-001 & $0.0220^{109}$ & $0.2254^{91}$ & $0.6184^{108}$ & - & - & - & - & - & - & - & - & - \\
\hline 75 & intelresearch-002 & $0.0111^{80}$ & $0.0458^{36}$ & $0.1598^{35}$ & $0.2085^{33}$ & $0.0384^{40}$ & $0.1025^{36}$ & $0.2568^{38}$ & $0.0598^{42}$ & $0.1764^{41}$ & $0.2583^{38}$ & $0.1857^{36}$ & $0.1595^{31}$ \\
\hline 76 & intsysmsu-002 & $0.0089^{63}$ & $0.0827^{60}$ & $0.3138^{72}$ & - & - & - & - & - & - & - & - & - \\
\hline 77 & iqface- 000 & $0.0128^{86}$ & $0.0885^{65}$ & $0.2867^{66}$ & - & - & - & - & - & - & - & - & - \\
\hline 78 & iqface-002 & $0.0086^{58}$ & $0.0193^{17}$ & $0.0445^{7}$ & $0.0725^{7}$ & $0.0184^{21}$ & $0.0317^{6}$ & $0.0671^{6}$ & $0.0215^{15}$ & $0.0643^{9}$ & $0.0997^{7}$ & $0.0633^{10}$ & $0.0448^{5}$ \\
\hline 79 & isap-002 & $0.0094^{65}$ & & $0.4090^{87}$ & - & - & - & - & - & - & - & - & - \\
\hline 80 & itmo-007 & $0.0098^{72}$ & $0.0840^{61}$ & $0.2685^{60}$ & - & - & - & - & - & - & - & - & - \\
\hline 81 & iws-000 & $0.6797^{143}$ & $0.9960^{118}$ & $0.9997^{144}$ & - & - & - & - & - & - & - & - & - \\
\hline 82 & kakao-003 & $0.0170^{101}$ & $0.1541^{85}$ & $0.4123^{88}$ & - & & & - & - & - & - & - & \\
\hline 83 & kedacom-000 & $0.0391^{120}$ & $0.3444^{97}$ & $0.6188^{109}$ & $0.6848^{63}$ & $0.2663^{59}$ & $0.5975^{60}$ & - & - & - & - & - & - \\
\hline 84 & kneron-005 & $0.0296^{115}$ & & $0.4567^{94}$ & . & & & - & 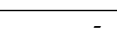 & - & - & - & \\
\hline 85 & kookmin-001 & $0.0357^{118}$ & $0.4479^{101}$ & $0.7378^{122}$ & $0.8497^{66}$ & $0.2933^{60}$ & $0.6006^{61}$ & - & $0.4160^{57}$ & $0.7365^{68}$ & $0.8917^{58}$ & $0.8642^{58}$ & $0.7432^{41}$ \\
\hline$\overline{86}$ & lookman-004 & $0.0398^{121}$ & ב- & $0.6520^{115}$ & $\overline{-1}$ & - & $\overline{-1}$ & $\overline{-1}$ & - & - & $\overline{-1}$ & $\overline{-1}$ & - \\
\hline 87 & luxand-000 & $0.2167^{136}$ & $0.9732^{115}$ & $0.9988^{140}$ & - & & & - & $\mathrm{C}_{\mathrm{s}}$ & -5 & - & - & \\
\hline 88 & $\mathrm{mt}-000$ & $0.0075^{51}$ & $0.0768^{55}$ & $0.2700^{61}$ & $0.3736^{50}$ & $0.0482^{43}$ & $0.1746^{46}$ & & $0.0749^{43}$ & $0.3084^{54}$ & $0.4239^{48}$ & - & \\
\hline 89 & $\mathrm{mt}-002$ & $0.0043^{15}$ & $0.0215^{18}$ & $0.0713^{18}$ & $0.1114^{17}$ & $0.0162^{12}$ & $0.0404^{15}$ & $0.0777^{12}$ & $0.0225^{17}$ & $0.0877^{19}$ & $0.1399^{18}$ & $0.0804^{15}$ & $0.0757^{14}$ \\
\hline 90 & mvision-001 & $0.0137^{92}$ & - & $0.3987^{85}$ & - & - & - & - & - & - & - & - & \\
\hline
\end{tabular}

Table 6: This table summarizes False Non-Match Rate (FNMR) on unmasked and masked probe images. FNMR is the proportion of mated comparisons below a threshold set to achieve FMR=1e-05 on unmasked probe images. False Match Rate (FMR) is the proportion of impostor comparisons at or above that threshold. The red superscripts give rank over all algorithms in that column. Missing entries generally mean the algorithm was not run on that particular mask variation due to time and resource constraints. Algorithms with FTE=1.00 were not run at all. Algorithms in black were submitted prior to mid-March 2020, and algorithms in blue were submitted thereafter. 


\begin{tabular}{|c|c|c|c|c|c|c|c|c|c|c|c|c|c|}
\hline & \multirow{3}{*}{$\begin{array}{l}\text { Algorithm } \\
\text { Name } \\
\text { COVERAGE }\end{array}$} & \multirow[t]{3}{*}{ NOT MASKED } & \multicolumn{6}{|c|}{ MASK COLOR = LIGHTBLUE } & \multirow{2}{*}{\multicolumn{3}{|c|}{$\begin{array}{c}\text { COLOR = BLACK } \\
\text { SHAPE }=\text { WIDE }\end{array}$}} & \multirow{3}{*}{$\begin{array}{r}\text { COLOR = RED } \\
\text { SHAPE = WIDE } \\
\text { MED }\end{array}$} & \multirow{3}{*}{$\begin{array}{r}\text { COLOR = WHITE } \\
\text { SHAPE = WIDE } \\
\text { MED }\end{array}$} \\
\hline & & & \multicolumn{3}{|c|}{ SHAPE $=$ WIDE } & \multicolumn{3}{|c|}{ SHAPE $=$ ROUND } & & & & & \\
\hline & & & LO & MED & $\mathrm{HI}$ & LO & MED & $\mathrm{HI}$ & LO & MED & $\mathrm{HI}$ & & \\
\hline 91 & nazhiai-000 & $0.0043^{16}$ & $0.0184^{13}$ & $0.0835^{21}$ & $0.1318^{20}$ & $0.0156^{11}$ & $0.0463^{20}$ & $0.0947^{18}$ & $0.0177^{10}$ & $0.0764^{16}$ & 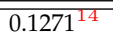 & $0.0792^{14}$ & $0.0854^{20}$ \\
\hline 92 & netbridgetech-001 & $0.2673^{139}$ & $0.8940^{112}$ & $0.9878^{137}$ & - & - & - & - & - & - & - & - & - \\
\hline 93 & netbridgetech-002 & $0.0083^{55}$ & $0.0781^{57}$ & $0.2723^{63}$ & $0.3522^{46}$ & $0.0528^{45}$ & $0.1551^{44}$ & - & $0.0875^{46}$ & $0.2863^{51}$ & $0.4151^{47}$ & - & - \\
\hline 94 & neurotechnology-008 & $0.0100^{73}$ & $0.0794^{58}$ & $0.3450^{77}$ & $0.4460^{57}$ & $0.0818^{51}$ & $0.1834^{48}$ & $0.3127^{40}$ & $0.0953^{48}$ & $0.4893^{60}$ & $0.5472^{52}$ & - & - \\
\hline 95 & neurotechnology-009 & $0.0051^{26}$ & $0.0351^{31}$ & $0.1768^{40}$ & $0.2537^{41}$ & $0.0268^{27}$ & $0.0953^{33}$ & $0.2034^{34}$ & $0.0414^{35}$ & $0.2180^{45}$ & $0.3192^{42}$ & $0.1658^{32}$ & - \\
\hline 96 & nodeflux-002 & $0.0424^{122}$ & $0.4177^{100}$ & $0.7307^{121}$ & 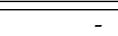 & - & 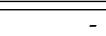 & 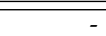 & 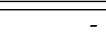 & - & 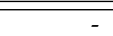 & - & - \\
\hline 97 & notiontag-000 & $0.6814^{144}$ & $0.9966^{119}$ & $0.9992^{142}$ & - & - & - & - & - & - & - & - & \\
\hline 98 & nsensecorp-001 & $1.0000^{151}$ & $1.0000^{126}$ & $1.0000^{151}$ & $1.0000^{74}$ & - & - & - & $1.0000^{67}$ & $1.0000^{78}$ & - & $1.0000^{60}$ & $1.0000^{44}$ \\
\hline 99 & ntechlab-008 & $0.0033^{2}$ & $0.0179^{12}$ & $0.0642^{16}$ & $0.1126^{18}$ & $0.0137^{8}$ & $0.0413^{18}$ & $0.0953^{19}$ & $0.0208^{14}$ & $0.0842^{18}$ & $0.1348^{17}$ & $0.0645^{11}$ & $0.0840^{19}$ \\
\hline 100 & oz-001 & $0.0152^{97}$ & $0.1969^{89}$ & $0.5887^{106}$ & - & - & - & - & - & - & - & - & - \\
\hline 101 & paravision-004 & $0.0088^{61}$ & $0.0124^{4}$ & $0.0281^{2}$ & $0.0476^{2}$ & $0.0125^{6}$ & $0.0181^{3}$ & $0.0313^{2}$ & $0.0135^{4}$ & $0.0327^{3}$ & $0.0581^{3}$ & $0.0332^{3}$ & $0.0313^{3}$ \\
\hline 102 & pensees-001 & $0.0106^{75}$ & $0.0309^{24}$ & $0.0461^{8}$ & $0.0921^{12}$ & $0.0326^{36}$ & $0.0413^{17}$ & $0.0893^{15}$ & $0.0333^{28}$ & $0.0579^{7}$ & $0.1217^{12}$ & $0.1338^{26}$ & $0.0462^{6}$ \\
\hline 103 & pixelall-003 & $0.0086^{59}$ & $0.0746^{52}$ & $0.2680^{58}$ & 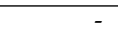 & & - & & - & & & 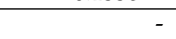 & \\
\hline 104 & pixelall-004 & $0.0078^{52}$ & $0.0524^{44}$ & $0.1676^{38}$ & $0.2141^{35}$ & $0.0495^{44}$ & $0.1131^{41}$ & $0.2254^{37}$ & $0.0849^{45}$ & $0.2461^{47}$ & $0.3158^{41}$ & $0.3148^{50}$ & $0.1791^{34}$ \\
\hline 105 & psl-005 & $0.0056^{34}$ & $0.0433^{35}$ & $0.1904^{47}$ & $0.2576^{42}$ & $0.0292^{30}$ & $0.1054^{39}$ & $0.2210^{36}$ & $0.0433^{37}$ & $0.1681^{39}$ & $0.2223^{34}$ & $0.1851^{35}$ & - \\
\hline 106 & ptakuratsatu-000 & $0.0059^{41}$ & $0.0542^{47}$ & $0.1897^{46}$ & $0.2485^{40}$ & $0.0380^{39}$ & $0.1047^{38}$ & - & $0.0502^{39}$ & $0.1652^{38}$ & $0.2360^{37}$ & $0.2003^{41}$ & - \\
\hline 107 & pxl-001 & $0.0511^{125}$ & $0.4879^{102}$ & $0.8183^{124}$ & $0.8754^{67}$ & $0.3675^{61}$ & $0.7276^{62}$ & $0.9182^{50}$ & $0.5416^{59}$ & $0.8047^{70}$ & $0.8718^{57}$ & - & - \\
\hline 108 & rankone-009 & $0.0065^{46}$ & $0.0951^{67}$ & $0.3450^{76}$ & $0.3709^{49}$ & $0.1004^{55}$ & $0.2244^{53}$ & $0.4467^{46}$ & - & $0.5624^{61}$ & $0.9343^{59}$ & - & - \\
\hline 109 & rankone-010 & $0.0065^{47}$ & $0.1514^{84}$ & $0.5452^{102}$ & $0.3915^{53}$ & - & - & - & - & $0.7196^{67}$ & - & $0.5811^{56}$ & $0.5719^{40}$ \\
\hline 110 & remarkai-002 & $0.0073^{49}$ & $0.0685^{50}$ & $0.2352^{53}$ & - & - & - & - & - & - & - & $0.2406^{46}$ & - \\
\hline 111 & rokid-000 & $0.0117^{82}$ & $0.1448^{81}$ & $0.4346^{91}$ & - & - & - & - & - & - & - & - & - \\
\hline 112 & s1-001 & $0.0277^{114}$ & $0.6776^{109}$ & $0.9459^{133}$ & - & - & - & - & - & - & - & - & - \\
\hline 113 & scanovate-001 & $0.2403^{138}$ & & $0.5973^{107}$ & - & & - & & & & & - & - \\
\hline 114 & scanovate-002 & $0.0119^{84}$ & $0.1304^{79}$ & $0.4006^{86}$ & $0.5142^{59}$ & $0.0757^{48}$ & $0.2206^{51}$ & $0.3622^{42}$ & $0.1215^{51}$ & $0.3172^{55}$ & $0.4633^{50}$ & - & - \\
\hline 115 & securifai-001 & $0.5185^{140}$ & $0.9381^{114}$ & $0.9835^{136}$ & $0.9880^{71}$ & $0.9146^{64}$ & $0.9788^{66}$ & - & $0.9352^{63}$ & $0.9853^{75}$ & $0.9949^{63}$ & $0.9867^{59}$ & $0.9829^{43}$ \\
\hline 116 & sensetime-003 & $0.0045^{21}$ & $0.0185^{14}$ & $0.0544^{11}$ & $0.0912^{11}$ & $0.0221^{23}$ & $0.0365^{13}$ & $0.0739^{11}$ & $0.0232^{20}$ & $0.0654^{11}$ & $0.1230^{13}$ & - & - \\
\hline 117 & sertis-000 & $0.0066^{48}$ & $0.0751^{53}$ & $0.2685^{59}$ & - & - & - & - & - & - & - & - & - \\
\hline 118 & sertis-001 & $0.0179^{102}$ & - & $0.5774^{104}$ & $0.6655^{61}$ & - & - & - & - & $0.5632^{62}$ & - & - & - \\
\hline 119 & shu-002 & $1.0000^{150}$ & & $1.0000^{149}$ & - & - & - & - & - & & - & - & - \\
\hline 120 & shu-003 & $0.0053^{28}$ & $0.0465^{37}$ & $0.1839^{45}$ & $0.2148^{36}$ & $0.0379^{38}$ & $0.1148^{43}$ & $0.2196^{35}$ & $0.0422^{36}$ & $0.1702^{40}$ & $0.2210^{33}$ & $0.1901^{37}$ & - \\
\hline$\overline{121}$ & $\begin{array}{c}\text { sjtu-002 } \\
\end{array}$ & $0.0052^{27}$ & $0.0475^{40}$ & $0.1912^{48}$ & - & - & - & - & - & & $\overline{-1}$ & $\overline{0.2090^{42}}$ & \\
\hline 122 & sjtu-003 & $0.0043^{14}$ & $0.0340^{30}$ & $0.1239^{29}$ & $0.1609^{26}$ & - & - & - & - & $0.1008^{24}$ & - & $0.1333^{25}$ & $0.1323^{27}$ \\
\hline 123 & staqu-000 & $0.0108^{77}$ & $0.1251^{77}$ & $0.3537^{79}$ & $0.4429^{55}$ & $0.0913^{53}$ & $0.2434^{55}$ & $0.4447^{45}$ & - & $0.3862^{58}$ & $0.6319^{53}$ & - & - \\
\hline 124 & starhybrid-001 & $0.0104^{74}$ & $0.1923^{88}$ & $0.5033^{98}$ & - & - & - & - & - & - & - & - & - \\
\hline 125 & synology-000 & $0.0123^{85}$ & - & $0.4459^{93}$ & - & - & - & - & - & - & - & - & - \\
\hline 126 & tech5-004 & $0.0045^{20}$ & $0.0218^{19}$ & $0.0839^{22}$ & $0.1389^{22}$ & $0.0172^{13}$ & $0.0464^{21}$ & $0.0905^{16}$ & $0.0228^{19}$ & $0.0818^{17}$ & $0.1288^{16}$ & $0.0826^{16}$ & $0.0830^{18}$ \\
\hline 127 & tech5-005 & $0.0037^{7}$ & $0.0224^{20}$ & $0.0941^{23}$ & $0.1518^{24}$ & $0.0180^{17}$ & $0.0524^{23}$ & $0.1066^{25}$ & $0.0254^{22}$ & $0.0986^{22}$ & $0.1571^{24}$ & $0.0979^{2 \sigma}$ & $0.0945^{21}$ \\
\hline 128 & tevian-005 & $0.0061^{43}$ & $0.0961^{68}$ & $0.5044^{100}$ & - & - & - & & - & $0.6178^{63}$ & & & \\
\hline 129 & tevian-006 & $0.0038^{10}$ & $0.0140^{7}$ & $0.0547^{12}$ & $0.0894^{9}$ & $0.0184^{20}$ & $0.0325^{7}$ & $0.1013^{22}$ & $0.0183^{11}$ & $0.1281^{30}$ & $0.1507^{20}$ & $0.1437^{27}$ & $0.0659^{13}$ \\
\hline 130 & trueface- 000 & $0.0143^{95}$ & $0.1512^{83}$ & $0.4164^{89}$ & - & - & - & - & - & - & - & - & - \\
\hline 131 & trueface-001 & $0.0097^{71}$ & $0.0978^{69}$ & $0.3483^{78}$ & $0.4310^{54}$ & $0.0710^{47}$ & $0.2064^{50}$ & $0.3846^{43}$ & - & $0.3752^{57}$ & $\overline{-1}$ & - & - \\
\hline 132 & tuputech-000 & $0.2014^{135}$ & $0.8743^{111}$ & $0.9731^{135}$ & - & - & - & - & - & - & - & - & - \\
\hline 133 & uluface- 002 & $0.0073^{50}$ & $0.0796^{59}$ & $0.2450^{54}$ & - & - & - & - & - & - & - & $0.3939^{54}$ & - \\
\hline 134 & upc-001 & $0.0167^{100}$ & & $0.4723^{95}$ & 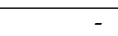 & & - & & - & & - & - & - \\
\hline 135 & veridas-004 & $0.0202^{107}$ & $0.2783^{94}$ & $0.6697^{116}$ & $0.7334^{65}$ & $0.1941^{58}$ & $0.5025^{59}$ & $0.6906^{49}$ & - & $0.6592^{66}$ & - & - & - \\
\hline
\end{tabular}

Table 7: This table summarizes False Non-Match Rate (FNMR) on unmasked and masked probe images. FNMR is the proportion of mated comparisons below a threshold set to achieve FMR=1e-05 on unmasked probe images. False Match Rate (FMR) is the proportion of impostor comparisons at or above that threshold. The red superscripts give rank over all algorithms in that column. Missing entries generally mean the algorithm was not run on that particular mask variation due to time and resource constraints. Algorithms with FTE=1.00 were not run at all. Algorithms in black were submitted prior to mid-March 2020, and algorithms in blue were submitted thereafter. 


\begin{tabular}{|c|c|c|c|c|c|c|c|c|c|c|c|c|c|}
\hline & Algorithm & NOT MASKED & \multicolumn{6}{|c|}{ MASK COLOR $=$ LIGHTBLUE } & \multirow{2}{*}{\multicolumn{3}{|c|}{$\begin{array}{c}\text { COLOR }=\text { BLACK } \\
\text { SHAPE }=\text { WIDE }\end{array}$}} & COLOR = RED & COLOR = WHITE \\
\hline & Name & & \multicolumn{3}{|c|}{ SHAPE $=$ WIDE } & \multicolumn{3}{|c|}{ SHAPE $=$ ROUND } & & & & SHAPE $=$ WIDE & SHAPE $=$ WIDE \\
\hline & COVERAGE & & LO & MED & $\mathrm{HI}$ & LO & MED & $\mathrm{HI}$ & $\mathrm{LO}$ & MED & $\mathrm{HI}$ & MED & MED \\
\hline$\overline{136}$ & $\begin{array}{l}\text { via-001 } \\
\end{array}$ & $\overline{0.0097^{70}}$ & $0.1234^{76}$ & $0.3406^{75}$ & 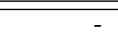 & - & $\overline{-1-}$ & $-\overline{-1}$ & $\overline{-1-}$ & - & - & - & - \\
\hline 137 & videmo-000 & $0.0140^{93}$ & - & $0.5509^{103}$ & - & - & - & - & - & - & - & - & - \\
\hline 138 & videonetics-002 & $0.6032^{141}$ & $0.9941^{117}$ & $0.9996^{143}$ & - & - & - & - & - & - & - & - & - \\
\hline 139 & vigilantsolutions- 007 & $0.0194^{104}$ & $0.2849^{95}$ & $0.6839^{120}$ & & - & - & - & - & - & - & - & - \\
\hline 140 & vigilantsolutions-008 & $0.0195^{105}$ & - & $0.4970^{97}$ & $0.5521^{60}$ & - & - & - & - & $0.6365^{65}$ & - & - & - \\
\hline 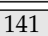 & vinai-000 & $0.0056^{33}$ & $\overline{0.0388^{33}}$ & $\overline{0.1587^{34}}$ & $0.2130^{34}$ & $0.0286^{29}$ & $0.0965^{34}$ & 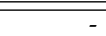 & $\overline{0.0364^{32}}$ & 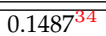 & $\overline{0.2024^{32}}$ & $0.1691^{33}$ & $\overline{0.1710^{33}}$ \\
\hline 142 & visionlabs-008 & $0.0034^{3}$ & $0.0139^{6}$ & $0.0579^{14}$ & $0.1014^{15}$ & $0.0154^{10}$ & $0.0412^{16}$ & $0.1004^{21}$ & $0.0187^{12}$ & $0.0664^{13}$ & $0.1284^{15}$ & $0.0614^{9}$ & $0.0618^{11}$ \\
\hline 143 & visionlabs-009 & $0.0028^{1}$ & $0.0095^{3}$ & $0.0355^{4}$ & $0.0667^{6}$ & $0.0123^{4}$ & $0.0307^{5}$ & $0.0719^{9}$ & $0.0123^{3}$ & $0.0423^{4}$ & $0.0943^{6}$ & $0.0399^{4}$ & $0.0395^{4}$ \\
\hline 144 & visteam-000 & $0.9960^{149}$ & $1.0000^{124}$ & $1.0000^{148}$ & - & - & - & - & - & - & - & - & - \\
\hline 145 & vocord-008 & $0.0038^{8}$ & $0.0140^{8}$ & $0.0500^{9}$ & $0.0762^{8}$ & $0.0176^{16}$ & $0.0393^{14}$ & $0.0892^{14}$ & $0.0135^{5}$ & $0.0459^{5}$ & $0.0771^{4}$ & $0.0607^{7}$ & $0.0482^{8}$ \\
\hline 146 & vts- 000 & $0.0199^{106}$ & $0.0870^{62}$ & $0.2755^{65}$ & $0.3566^{47}$ & 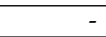 & - & - & 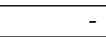 & $0.2584^{50}$ & - & $0.2249^{43}$ & $0.2976^{38}$ \\
\hline 147 & winsense-001 & $0.0058^{38}$ & $0.0473^{39}$ & $0.1626^{36}$ & $0.2244^{37}$ & $0.0325^{35}$ & $0.0946^{32}$ & $0.1853^{32}$ & $0.0406^{33}$ & $0.1471^{33}$ & $0.2231^{35}$ & $0.1622^{31}$ & $0.1843^{35}$ \\
\hline 148 & x-laboratory-001 & $0.0058^{39}$ & $0.0517^{43}$ & $0.2569^{56}$ & & & - & - & & & - & $0.2333^{44}$ & \\
\hline 149 & xforwardai- 000 & $0.0056^{32}$ & $0.0235^{22}$ & $0.1064^{27}$ & $0.1615^{27}$ & $0.0197^{22}$ & $0.0606^{27}$ & $0.1156^{27}$ & $0.0255^{23}$ & $0.1091^{26}$ & $0.1608^{26}$ & $0.1108^{22}$ & $0.1101^{24}$ \\
\hline 150 & xforwardai-001 & $0.0041^{12}$ & $0.0087^{2}$ & $0.0289^{3}$ & $0.0536^{3}$ & $0.0087^{2}$ & $0.0180^{2}$ & $0.0377^{3}$ & $0.0090^{2}$ & $0.0303^{2}$ & $0.0544^{2}$ & $0.0313^{2}$ & $0.0294^{2}$ \\
\hline 151 & $\mathrm{xm}-000$ & $0.0044^{17}$ & $0.0334^{29}$ & $0.1255^{30}$ & $0.1682^{28}$ & $0.0275^{28}$ & $0.0774^{29}$ & $0.1648^{30}$ & $0.0324^{26}$ & $0.1274^{29}$ & $0.1839^{29}$ & $0.1706^{34}$ & $0.1381^{28}$ \\
\hline 152 & yoonik-000 & $0.0520^{127}$ & $0.1056^{73}$ & $0.2675^{57}$ & $0.3773^{51}$ & $0.0895^{52}$ & $0.1823^{47}$ & $0.3214^{41}$ & $0.1027^{49}$ & $0.2582^{49}$ & $0.3628^{44}$ & - & - \\
\hline 153 & yuan- 000 & $0.0130^{88}$ & $0.0871^{63}$ & $0.2704^{62}$ & $0.3516^{45}$ & $0.0654^{46}$ & $0.1661^{45}$ & - & $0.0805^{44}$ & $0.2492^{48}$ & $0.3645^{45}$ & - & - \\
\hline
\end{tabular}

Table 8: This table summarizes False Non-Match Rate (FNMR) on unmasked and masked probe images. FNMR is the proportion of mated comparisons below a threshold set to achieve FMR=1e-05 on unmasked probe images. False Match Rate (FMR) is the proportion of impostor comparisons at or above that threshold. The red superscripts give rank over all algorithms in that column. Missing entries generally mean the algorithm was not run on that particular mask variation due to time and resource constraints. Algorithms with FTE=1.00 were not run at all. Algorithms in black were submitted prior to mid-March 2020, and algorithms in blue were submitted thereafter. 


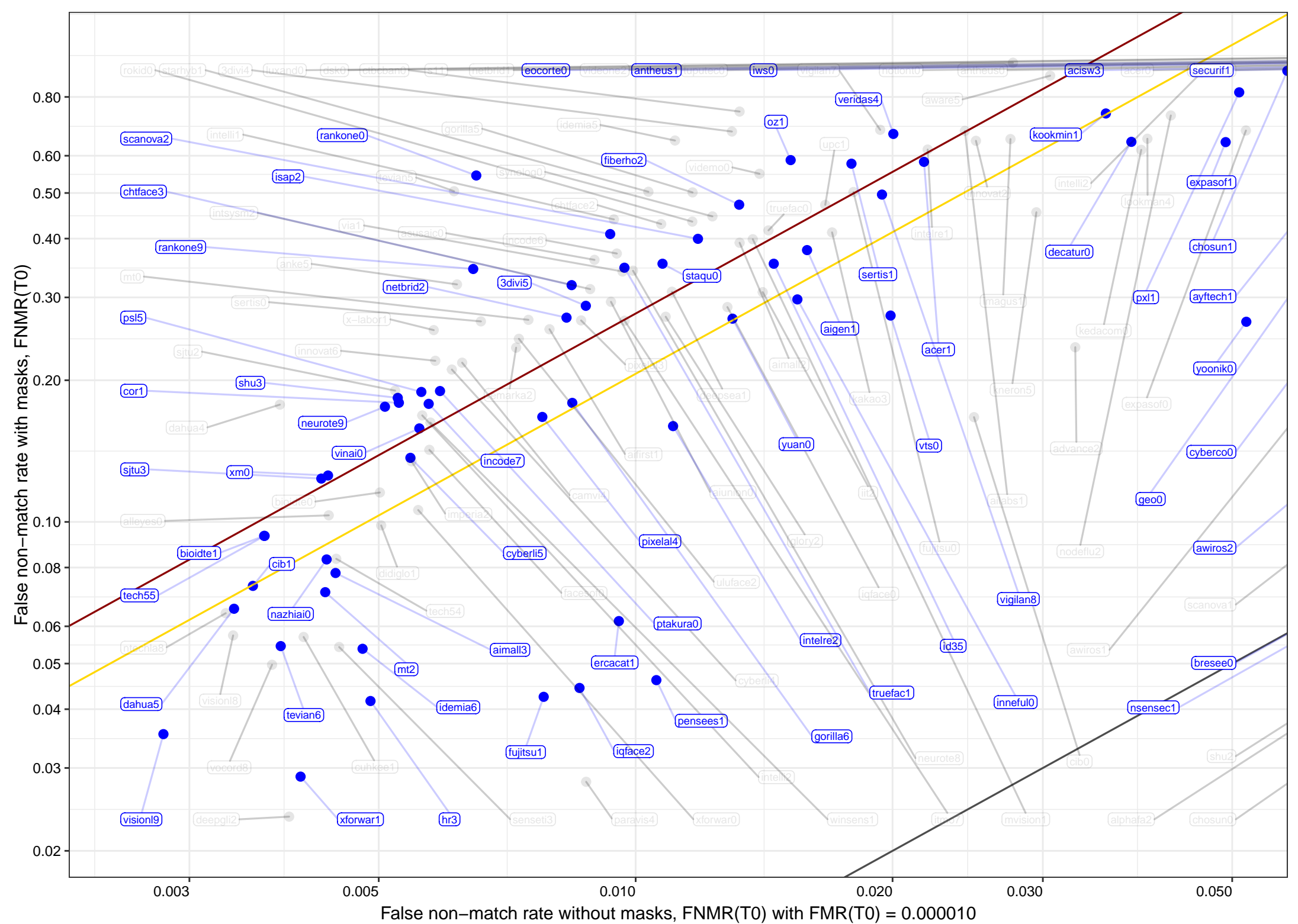

Figure 7: At a fixed threshold, a plot of FNMR with and without masks. The displacement of the dark red line relative to the black "parity" line shows a large increase in FNMR with masks for pre-COVID algorithms. The reduction in distance (relative to the black line) observed in the gold line indicates a reduction in median FNMR with masks for post-COVID algorithms. The value in the title is the median increase multiplier. 
Impact of wide vs. round shape for medium lightblue masks

The lower gray line is $y=x$; the upper dark red line is $y=1.7 x$ (Pre-COVID); the middle gold line is $y=1.6 x$ (Post-COVID) $\|$ where $x$ is the median increase multiplier

Algorithm Submission

Post-COVID

- Pre-COVID

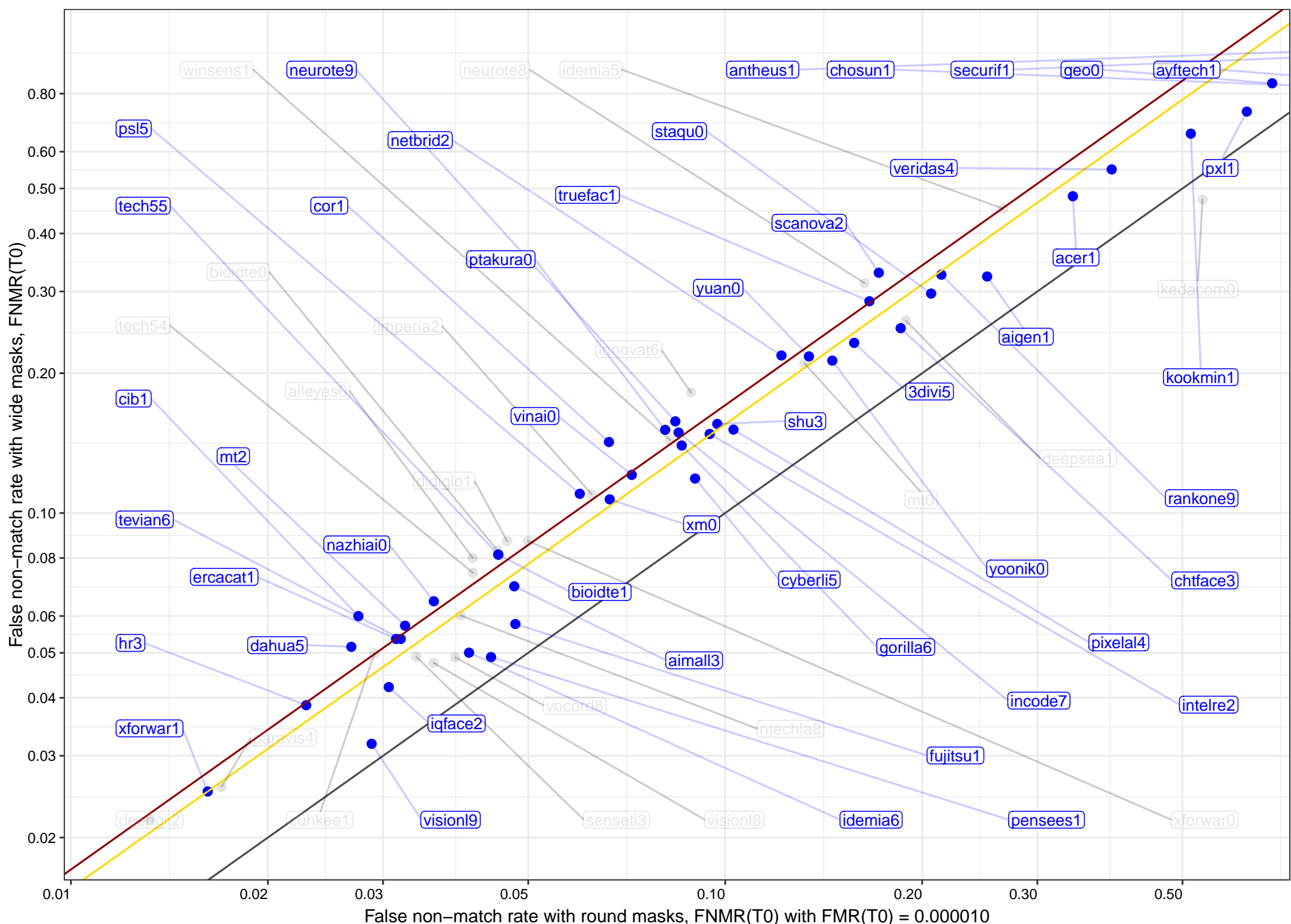

Figure 8: At a fixed threshold, a plot of FNMR with round versus wide masks. The displacement of the dark red (pre-COVID algorithms) and gold (post-COVID algorithms) lines relative to the black "parity" line shows a modest increase in FNMR with wide masks, with median post-COVID results showing nominal FNMR reductions. The value in the title is the median increase multiplier 
Impact of high vs. low nose coverage for wide lightblue masks

The lower gray line is $y=x$; the upper dark red line is $y=5.8 x$ (Pre-COVID); the middle gold line is $y=4.6 x$ (Post-COVID) $\|$ where $x$ is the median increase multiplier

Algorithm Submission

Post-COVID

- Pre-COVID

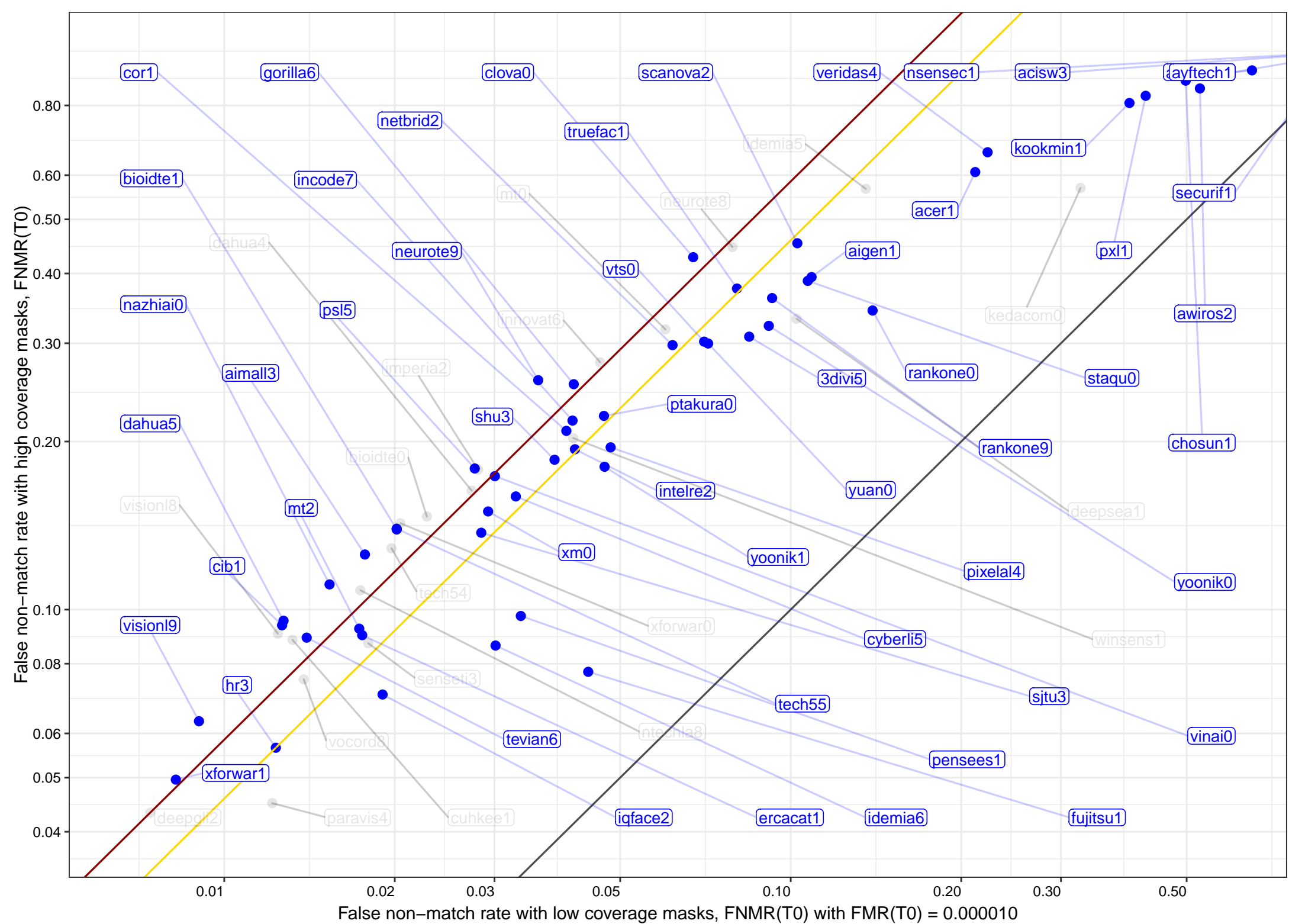

Figure 9: At a fixed threshold, a plot of FNMR with low versus high masks. The displacement of the dark red (pre-COVID algorithms) and gold (post-COVID algorithms) lines relative to the black "parity" lines shows a considerable increase in FNMR with high vs. low nose coverage masks, with median post-COVID results showing modest FNMR reductions. The value in the title is the median increase multiplier. 


\begin{tabular}{|c|c|c|c|c|c|c|c|c|c|c|c|c|c|c|c|c|}
\hline & \multirow{3}{*}{$\begin{array}{l}\text { Algorithm } \\
\text { Name } \\
\text { COVERAGE }\end{array}$} & \multirow{2}{*}{\multicolumn{3}{|c|}{$\begin{array}{c}\text { COLOR }=\text { WHITE } \\
\text { SHAPE }=\text { WIDE }\end{array}$}} & \multicolumn{6}{|c|}{ COLOR $=$ LIGHTBLUE } & \multirow{2}{*}{\multicolumn{3}{|c|}{$\begin{array}{l}\text { COLOR = RED } \\
\text { SHAPE = WIDE }\end{array}$}} & \multirow{2}{*}{\multicolumn{3}{|c|}{$\begin{array}{c}\text { COLOR }=\text { BLACK } \\
\text { SHAPE }=\text { WIDE }\end{array}$}} \\
\hline & & & & & \multicolumn{3}{|c|}{ SHAPE $=$ WIDE } & \multicolumn{3}{|c|}{ SHAPE $=$ ROUND } & & & & & & \\
\hline & & LO & MED & $\overline{\mathrm{HI}}$ & LO & MED & $\overline{\mathrm{HI}}$ & LO & MED & $\overline{\mathrm{HI}}$ & LO & MED & $\overline{\mathrm{HI}}$ & LO & MED & $\mathrm{HI}$ \\
\hline 1 & 3divi-004 & 0.514 & 0.659 & 0.627 & 0.420 & 0.599 & $\overline{0.603}$ & 0.378 & 0.663 & 0.769 & 0.290 & 0.691 & 0.864 & 0.653 & 0.920 & 0.939 \\
\hline$\frac{1}{2}$ & 3divi-005 & 0.003 & 0.012 & 0.029 & 0.002 & 0.013 & 0.030 & 0.007 & 0.011 & 0.026 & 0.005 & 0.040 & 0.077 & 0.002 & 0.006 & 0.012 \\
\hline 3 & acer-000 & 0.048 & 0.105 & 0.139 & 0.035 & 0.080 & 0.114 & 0.052 & 0.078 & 0.137 & 0.168 & 0.321 & 0.445 & 0.107 & 0.197 & 0.270 \\
\hline 4 & acer-001 & 0.020 & 0.048 & 0.091 & 0.019 & 0.051 & 0.096 & 0.025 & 0.034 & 0.060 & 0.025 & 0.080 & 0.147 & 0.026 & 0.088 & 0.157 \\
\hline 5 & acisw-003 & 0.000 & 0.000 & 0.000 & 0.000 & 0.000 & 0.000 & 0.000 & 0.000 & 0.000 & 0.000 & 0.000 & 0.000 & 0.000 & 0.000 & 0.000 \\
\hline 6 & advance-002 & 0.019 & 0.046 & 0.096 & 0.020 & 0.045 & $\overline{0.096}$ & 0.026 & 0.037 & 0.085 & 0.028 & 0.088 & 0.174 & 0.034 & 0.104 & $\overline{0.200}$ \\
\hline 7 & aifirst-001 & 0.000 & 0.000 & 0.000 & 0.000 & 0.000 & 0.000 & 0.000 & 0.000 & 0.000 & 0.000 & 0.000 & 0.000 & 0.000 & 0.000 & 0.000 \\
\hline 8 & aigen-001 & 0.000 & 0.000 & 0.000 & 0.000 & 0.000 & 0.000 & 0.000 & 0.000 & 0.000 & 0.000 & 0.000 & 0.000 & 0.000 & 0.000 & 0.000 \\
\hline 9 & ailabs-001 & 0.071 & 0.208 & 0.248 & 0.061 & 0.194 & 0.233 & 0.102 & 0.177 & 0.314 & 0.096 & 0.319 & 0.000 & 0.116 & 0.310 & 0.465 \\
\hline 10 & aimall-002 & 0.073 & 0.129 & 0.225 & 0.095 & 0.152 & 0.260 & 0.107 & 0.159 & 0.236 & 0.086 & 0.122 & 0.230 & 0.049 & 0.071 & 0.154 \\
\hline 11 & בaimall-003 & 0.011 & 0.038 & 0.097 & 0.014 & 0.047 & $\overline{0.113}$ & 0.020 & 0.041 & 0.085 & 0.013 & 0.039 & 0.103 & 0.012 & 0.033 & $\overline{0.081}$ \\
\hline 12 & aiunionface-000 & 0.000 & 0.000 & 0.000 & 0.000 & 0.000 & 0.000 & 0.000 & 0.000 & 0.000 & 0.000 & 0.000 & 0.000 & 0.000 & 0.000 & 0.000 \\
\hline 13 & alleyes-000 & 0.006 & 0.023 & 0.062 & 0.006 & 0.020 & 0.056 & 0.007 & 0.012 & 0.028 & 0.007 & 0.035 & 0.088 & 0.010 & 0.043 & 0.104 \\
\hline 14 & alphaface-002 & 0.025 & 0.056 & 0.099 & 0.024 & 0.054 & 0.095 & 0.033 & 0.044 & 0.072 & 0.041 & 0.104 & 0.174 & 0.027 & 0.071 & 0.132 \\
\hline 15 & anke-005 & 0.009 & 0.028 & 0.066 & 0.011 & 0.030 & 0.069 & 0.012 & 0.018 & 0.041 & 0.013 & 0.036 & 0.079 & 0.009 & 0.056 & 0.091 \\
\hline 16 & antheus-000 & 0.000 & 0.000 & 0.000 & 0.000 & 0.000 & 0.000 & 0.000 & 0.000 & 0.000 & 0.000 & 0.000 & 0.000 & 0.000 & 0.000 & 0.000 \\
\hline 17 & antheus-001 & 0.000 & 0.000 & 0.000 & 0.000 & 0.000 & 0.000 & 0.000 & 0.000 & 0.000 & 0.000 & 0.000 & 0.000 & 0.000 & 0.000 & 0.000 \\
\hline 18 & asusaics-000 & 0.000 & 0.000 & 0.000 & 0.000 & 0.000 & 0.000 & 0.000 & 0.000 & 0.000 & 0.000 & 0.000 & 0.000 & 0.000 & 0.000 & 0.000 \\
\hline 19 & aware-005 & 0.053 & 0.151 & 0.218 & 0.039 & 0.129 & 0.211 & 0.046 & 0.089 & 0.244 & 0.095 & 0.380 & 0.516 & 0.091 & 0.236 & 0.449 \\
\hline 20 & awiros-001 & 0.195 & 0.370 & 0.450 & 0.162 & 0.298 & 0.379 & 0.161 & 0.258 & 0.355 & 0.388 & 0.650 & 0.772 & 0.198 & 0.415 & 0.642 \\
\hline 21 & awiros-002 & 0.101 & 0.212 & 0.215 & 0.103 & 0.202 & $\overline{0.220}$ & 0.187 & 0.261 & 0.356 & 0.071 & 0.272 & 0.296 & 0.232 & 0.405 & $\overline{0.455}$ \\
\hline 22 & ayftech-001 & 0.237 & 0.610 & 0.587 & 0.251 & 0.615 & 0.609 & 0.301 & 0.553 & 0.728 & 0.105 & 0.405 & 0.626 & 0.193 & 0.596 & 0.758 \\
\hline 23 & bioidtechswiss-000 & 0.005 & 0.022 & 0.061 & 0.006 & 0.028 & 0.070 & 0.010 & 0.021 & 0.046 & 0.006 & 0.025 & 0.068 & 0.006 & 0.021 & 0.058 \\
\hline 24 & bioidttechswiss-001 & 0.004 & 0.014 & 0.044 & 0.004 & 0.017 & 0.046 & 0.006 & 0.011 & 0.026 & 0.005 & 0.020 & 0.051 & 0.007 & 0.022 & 0.056 \\
\hline 25 & bresee-000 & 0.005 & 0.022 & 0.049 & 0.005 & 0.020 & 0.049 & 0.009 & 0.014 & 0.034 & 0.010 & 0.051 & 0.108 & 0.008 & 0.041 & 0.097 \\
\hline 26 & camvi-004 & 0.000 & 0.000 & 0.000 & 0.000 & 0.000 & 0.000 & 0.000 & 0.000 & 0.000 & 0.000 & 0.000 & 0.000 & 0.000 & 0.000 & 0.000 \\
\hline 27 & chosun-000 & 0.000 & 0.000 & 0.000 & 0.000 & 0.000 & 0.000 & 0.000 & 0.000 & 0.000 & 0.000 & 0.000 & 0.000 & 0.000 & 0.000 & 0.000 \\
\hline 28 & chosun-001 & 0.000 & 0.000 & 0.000 & 0.000 & 0.000 & 0.000 & 0.000 & 0.000 & 0.000 & 0.000 & 0.000 & 0.000 & 0.000 & 0.000 & 0.000 \\
\hline 29 & chtface-002 & 0.033 & 0.100 & 0.154 & 0.026 & 0.081 & 0.126 & 0.031 & 0.056 & 0.107 & 0.110 & 0.321 & 0.480 & 0.042 & 0.144 & 0.270 \\
\hline 30 & chtface-003 & 0.022 & 0.075 & 0.125 & 0.018 & 0.059 & 0.099 & $\begin{array}{l}0.0013 \\
0.023\end{array}$ & $\begin{array}{l}0.039 \\
0.039\end{array}$ & $\begin{array}{l}0.1077 \\
0.077\end{array}$ & 0.080 & 0.0259 & $\begin{array}{l}0.400 \\
0.418\end{array}$ & 0.030 & $\begin{array}{l}0.147 \\
0.108\end{array}$ & 0.224 \\
\hline 31 & cib-000 & 0.000 & $\overline{0.000}$ & 0.000 & 0.000 & 0.000 & $\overline{0.000}$ & 0.000 & $\overline{0.000}$ & 0.000 & 0.000 & 0.000 & 0.000 & 0.000 & $\overline{0.000}$ & $\overline{0.000}$ \\
\hline 32 & cib-001 & 0.000 & 0.000 & 0.000 & 0.000 & 0.000 & 0.000 & 0.000 & 0.000 & 0.000 & 0.000 & 0.000 & 0.000 & 0.000 & 0.000 & 0.000 \\
\hline 33 & clova-000 & 0.035 & 0.089 & 0.149 & 0.033 & 0.081 & 0.136 & 0.039 & 0.059 & 0.128 & 0.071 & 0.245 & 0.361 & 0.049 & 0.146 & 0.252 \\
\hline 34 & cor-001 & 0.002 & 0.010 & 0.031 & 0.003 & 0.013 & 0.035 & 0.004 & 0.008 & 0.020 & 0.004 & 0.013 & 0.032 & 0.004 & 0.011 & 0.031 \\
\hline 35 & ctbcbank-000 & 0.179 & 0.794 & 0.803 & 0.171 & 0.786 & 0.865 & 0.205 & 0.620 & 0.915 & 0.433 & 0.857 & 0.913 & 0.189 & 0.806 & 0.895 \\
\hline 36 & cuhkee-001 & 0.009 & 0.029 & 0.069 & 0.009 & 0.031 & $\overline{0.074}$ & 0.014 & 0.025 & 0.057 & 0.012 & 0.048 & 0.115 & 0.013 & $\overline{0.048}$ & $\overline{0.140}$ \\
\hline 37 & cybercore- 000 & 0.194 & 0.376 & 0.371 & 0.222 & 0.447 & 0.453 & 0.267 & 0.461 & 0.751 & 0.191 & 0.432 & 0.554 & 0.367 & 0.678 & 0.805 \\
\hline 38 & cyberlink-004 & 0.014 & 0.042 & 0.096 & 0.013 & 0.039 & 0.091 & 0.018 & 0.029 & 0.063 & 0.020 & 0.096 & 0.177 & 0.018 & 0.064 & 0.136 \\
\hline 39 & cyberlink-005 & 0.012 & 0.035 & 0.080 & 0.011 & 0.029 & 0.067 & 0.014 & 0.021 & 0.043 & 0.016 & 0.072 & 0.147 & 0.015 & 0.049 & 0.114 \\
\hline 40 & dahua-004 & 0.033 & 0.150 & 0.087 & 0.027 & 0.126 & 0.094 & 0.047 & 0.121 & 0.190 & 0.012 & 0.071 & 0.187 & 0.011 & 0.057 & 0.183 \\
\hline 41 & dahua-005 & 0.000 & 0.000 & 0.000 & 0.000 & 0.000 & 0.000 & 0.000 & 0.000 & 0.000 & 0.000 & 0.000 & 0.000 & 0.000 & 0.000 & 0.000 \\
\hline 42 & decatur-000 & 0.014 & 0.036 & 0.074 & 0.014 & 0.033 & 0.069 & 0.018 & 0.028 & 0.058 & 0.032 & 0.065 & 0.135 & 0.033 & 0.069 & 0.135 \\
\hline 43 & deepglint-002 & 0.002 & 0.009 & 0.028 & 0.002 & 0.012 & 0.031 & 0.004 & 0.006 & 0.017 & 0.003 & 0.014 & 0.035 & 0.003 & 0.010 & 0.024 \\
\hline 44 & deepsea-001 & 0.000 & 0.000 & 0.000 & 0.000 & 0.000 & 0.000 & 0.000 & 0.000 & 0.000 & 0.000 & 0.000 & 0.000 & 0.000 & 0.000 & 0.000 \\
\hline 45 & didiglobalface-001 & 0.025 & 0.056 & 0.099 & 0.024 & 0.054 & 0.095 & 0.033 & 0.044 & 0.072 & 0.041 & 0.104 & 0.174 & 0.027 & 0.071 & 0.132 \\
\hline
\end{tabular}

Table 9: This table summarizes Failure to Enroll (FTE) rates surveyed over 10000 images of each mask variant. FTE is the proportion of failed template generation attempts. Failures can occur because the software throws an exception, or because the software electively refuses to process the input image as would occur if the algorithms does not detect a face or determines that the face has insufficient information. FTE is measured as the number of function calls that give EITHER a non-zero error code OR that give a "small" template containing fewer than 60 bytes. This second rule is needed because some algorithms incorrectly fail to return a non-zero error code when template generation fails but do produce a skeletal template. The effects of FTE are included in the accuracy results of this report by regarding any template comparison involving a failed template to produce a low similarity score. Thus higher FTE results in higher FNMR and lower FMR. Algorithms in black were submitted prior to mid-March 2020, and algorithms in blue were submitted thereafter. 


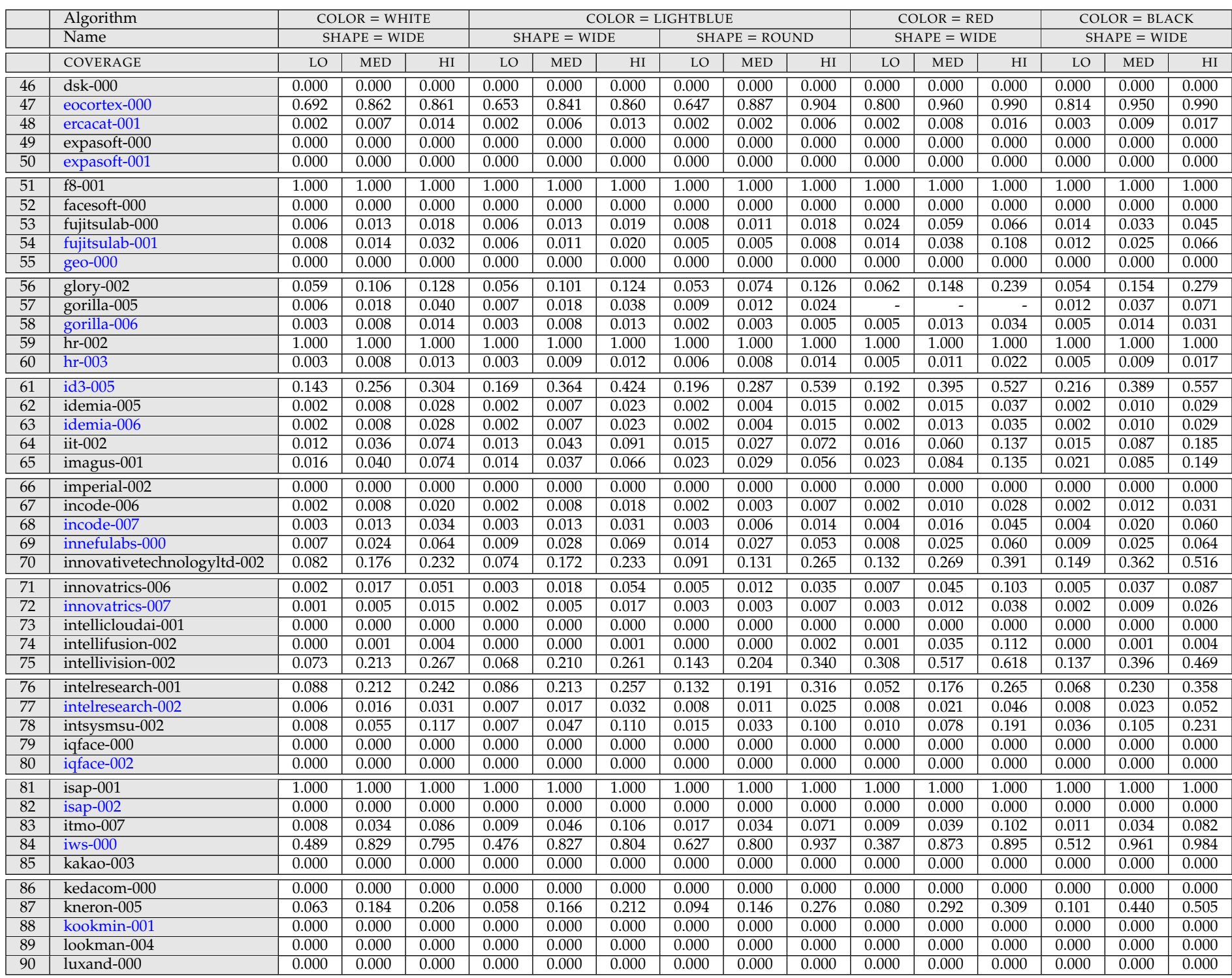

Table 10: This table summarizes Failure to Enroll (FTE) rates surveyed over 10000 images of each mask variant. FTE is the proportion of failed template generation attempts. Failures can occur because the software throws an exception, or because the software electively refuses to process the input image as would occur if the algorithms does not detect a face or determines that the face has insufficient information. FTE is measured as the number of function calls that give EITHER a non-zero error code OR that give a "small" template containing fewer than 60 bytes. This second rule is needed because some algorithms incorrectly fail to return a non-zero error code when template generation fails but do produce a skeletal template. The effects of FTE are included in the accuracy results of this report by regarding any template comparison involving a failed template to produce a low similarity score. Thus higher FTE results in higher FNMR and lower FMR. Algorithms in black were submitted prior to mid-March 2020, and algorithms in blue were submitted thereafter. 


\begin{tabular}{|c|c|c|c|c|c|c|c|c|c|c|c|c|c|c|c|c|}
\hline & \multirow{2}{*}{$\begin{array}{l}\text { Algorithm } \\
\text { Name } \\
\text { COVERAGE } \\
\end{array}$} & \multicolumn{3}{|c|}{$\begin{array}{l}\text { COLOR }=\text { WHITE } \\
\text { SHAPE }=\text { WIDE }\end{array}$} & \multicolumn{6}{|c|}{ COLOR = LIGHTBLUE } & \multicolumn{3}{|c|}{$\begin{array}{l}\text { COLOR = RED } \\
\text { SHAPE = WIDE }\end{array}$} & \multicolumn{3}{|c|}{$\begin{array}{l}\text { COLOR }=\text { BLACK } \\
\text { SHAPE }=\text { WIDE }\end{array}$} \\
\hline & & $\overline{\mathrm{LO}}$ & MED & $\mathrm{HI}$ & $\mathrm{LO}$ & MED & $\mathrm{HI}$ & $\mathrm{LO}$ & MED & $\mathrm{HI}$ & LO & MED & $\mathrm{HI}$ & $\mathrm{LO}$ & MED & $\mathrm{HI}$ \\
\hline 91 & mobai-000 & 0.186 & 0.306 & 0.323 & 0.200 & 0.299 & 0.329 & 0.204 & 0.275 & 0.386 & 0.403 & 0.643 & 0.755 & 0.219 & 0.397 & 0.527 \\
\hline 92 & $\mathrm{mt}-000$ & 0.005 & 0.021 & 0.061 & 0.006 & 0.024 & 0.063 & 0.011 & 0.021 & 0.045 & 0.006 & 0.024 & 0.058 & 0.007 & 0.023 & 0.059 \\
\hline 93 & $\mathrm{mt}-002$ & 0.001 & 0.004 & 0.007 & 0.001 & 0.004 & 0.006 & 0.001 & 0.001 & 0.003 & 0.001 & 0.005 & 0.013 & 0.002 & 0.005 & 0.009 \\
\hline 94 & mvision-001 & 0.000 & 0.000 & 0.000 & 0.000 & 0.000 & 0.000 & 0.000 & 0.000 & 0.000 & 0.000 & 0.000 & 0.000 & 0.000 & 0.000 & 0.000 \\
\hline 95 & nazhiai-000 & 0.000 & 0.000 & 0.000 & 0.000 & 0.000 & 0.000 & 0.000 & 0.000 & 0.000 & 0.000 & 0.000 & 0.000 & 0.000 & 0.000 & 0.000 \\
\hline 96 & netbridgetech-001 & 0.000 & 0.000 & 0.000 & 0.000 & 0.000 & 0.000 & 0.000 & 0.000 & 0.000 & 0.000 & 0.000 & 0.000 & 0.000 & 0.000 & 0.000 \\
\hline 97 & netbridgetech-002 & 0.000 & 0.000 & 0.000 & 0.000 & 0.000 & 0.000 & 0.000 & 0.000 & 0.000 & 0.000 & 0.000 & 0.000 & 0.000 & 0.000 & 0.000 \\
\hline 98 & neurotechnology-008 & 0.008 & 0.029 & 0.035 & 0.007 & 0.025 & 0.032 & 0.007 & 0.010 & 0.020 & 0.007 & 0.047 & 0.041 & 0.019 & 0.107 & 0.082 \\
\hline 99 & neurotechnology-009 & 0.006 & 0.018 & 0.026 & 0.005 & 0.015 & 0.023 & 0.004 & 0.005 & 0.014 & 0.009 & 0.030 & 0.043 & 0.015 & 0.100 & 0.086 \\
\hline 100 & nodeflux-002 & 0.402 & 0.598 & 0.538 & 0.440 & 0.671 & 0.628 & 0.482 & 0.681 & 0.877 & 0.393 & 0.672 & 0.772 & 0.602 & 0.835 & 0.915 \\
\hline 101 & notiontag-000 & 0.000 & 0.000 & 0.000 & 0.000 & 0.000 & 0.000 & 0.000 & 0.000 & 0.000 & 0.000 & 0.000 & 0.000 & 0.000 & 0.000 & 0.000 \\
\hline 102 & nsensecorp-001 & 0.074 & 0.139 & 0.196 & 0.089 & 0.174 & 0.241 & 0.073 & 0.119 & 0.264 & 0.073 & 0.137 & 0.204 & 0.083 & 0.219 & 0.291 \\
\hline 103 & ntechlab-008 & 0.064 & 0.126 & 0.196 & 0.053 & 0.011 & 0.183 & 0.003 & 0.095 & 0.018 & 0.003 & 0.016 & 0.043 & 0.003 & 0.016 & 0.042 \\
\hline 104 & oz-001 & 0.010 & 0.030 & 0.057 & 0.010 & 0.034 & 0.066 & 0.024 & 0.031 & 0.063 & 0.036 & 0.164 & 0.209 & 0.007 & 0.027 & 0.040 \\
\hline 105 & paravision-004 & 0.002 & 0.011 & 0.027 & 0.002 & 0.010 & 0.024 & 0.003 & 0.004 & 0.009 & 0.003 & 0.019 & 0.056 & 0.003 & 0.016 & 0.043 \\
\hline 106 & pensees-001 & 0.000 & 0.000 & 0.000 & 0.000 & 0.000 & 0.000 & 0.000 & 0.000 & 0.000 & 0.000 & 0.000 & 0.000 & 0.000 & 0.000 & 0.000 \\
\hline 107 & pixelall-003 & 0.000 & 0.000 & 0.000 & 0.000 & 0.000 & 0.000 & 0.000 & 0.000 & 0.000 & 0.000 & 0.000 & 0.000 & 0.000 & 0.000 & 0.000 \\
\hline 108 & pixelall-004 & 0.000 & 0.000 & 0.000 & 0.000 & 0.000 & 0.000 & 0.000 & 0.000 & 0.000 & 0.000 & 0.000 & 0.000 & 0.000 & 0.000 & 0.000 \\
\hline 109 & psl-005 & 0.004 & 0.015 & 0.038 & 0.003 & 0.014 & 0.036 & 0.006 & 0.011 & 0.029 & 0.006 & 0.025 & 0.061 & 0.008 & 0.020 & 0.047 \\
\hline 110 & ptakuratsatu- 000 & 0.001 & 0.005 & 0.015 & 0.002 & 0.005 & 0.017 & 0.003 & 0.003 & 0.007 & 0.003 & 0.012 & 0.038 & 0.002 & 0.009 & 0.026 \\
\hline 111 & pxl-001 & 0.096 & 0.268 & 0.329 & 0.082 & 0.208 & 0.273 & 0.090 & 0.161 & 0.294 & 0.272 & 0.579 & 0.712 & 0.116 & 0.302 & 0.500 \\
\hline 112 & rankone-009 & 0.000 & 0.000 & 0.000 & 0.000 & 0.000 & 0.000 & 0.000 & 0.000 & 0.000 & 0.000 & 0.000 & 0.001 & 0.000 & 0.000 & 0.001 \\
\hline 113 & rankone- 010 & 0.000 & 0.000 & 0.000 & 0.000 & 0.000 & 0.000 & 0.000 & 0.000 & 0.000 & 0.000 & 0.000 & 0.000 & 0.000 & 0.000 & 0.000 \\
\hline 114 & remarkai-002 & 0.000 & 0.000 & 0.000 & 0.000 & 0.000 & 0.000 & 0.000 & 0.000 & 0.000 & 0.000 & 0.000 & 0.000 & 0.000 & 0.000 & 0.000 \\
\hline 115 & rokid-000 & 0.194 & 0.372 & 0.370 & 0.220 & 0.444 & 0.450 & 0.265 & 0.457 & 0.749 & 0.191 & 0.431 & 0.553 & 0.367 & 0.677 & 0.806 \\
\hline 116 & s1-001 & 0.647 & 0.943 & 0.911 & 0.617 & 0.930 & 0.915 & 0.616 & 0.919 & 0.954 & 0.613 & 0.957 & 0.960 & 0.646 & 0.962 & 0.962 \\
\hline 117 & scanovate-001 & 0.544 & 0.601 & 0.596 & 0.515 & 0.553 & 0.579 & 0.513 & 0.565 & 0.664 & 0.766 & 0.914 & 0.960 & 0.554 & 0.676 & 0.806 \\
\hline 118 & scanovate- 002 & 0.008 & 0.019 & 0.046 & 0.008 & 0.018 & 0.045 & 0.008 & 0.012 & 0.027 & 0.010 & 0.025 & 0.079 & 0.012 & 0.036 & 0.101 \\
\hline 119 & securifai-001 & 0.000 & 0.000 & 0.000 & 0.000 & 0.000 & 0.000 & 0.000 & 0.000 & 0.000 & 0.000 & 0.000 & 0.000 & 0.000 & 0.000 & 0.000 \\
\hline 120 & $\begin{array}{l}\text { sensetime- } 003 \\
\end{array}$ & 0.009 & 0.029 & 0.069 & 0.009 & 0.031 & 0.074 & 0.014 & 0.025 & 0.057 & 0.012 & 0.048 & 0.115 & 0.013 & 0.048 & 0.140 \\
\hline 121 & sertis-000 & 0.002 & 0.012 & 0.034 & 0.002 & 0.012 & 0.032 & 0.003 & 0.005 & 0.013 & 0.003 & 0.016 & 0.047 & 0.005 & 0.020 & 0.052 \\
\hline 122 & sertis-001 & 0.002 & 0.006 & 0.017 & 0.002 & 0.006 & 0.014 & 0.001 & 0.002 & 0.005 & 0.002 & 0.007 & 0.017 & 0.003 & 0.009 & 0.020 \\
\hline 123 & shu-002 & 0.011 & 0.031 & 0.080 & 0.009 & 0.026 & 0.083 & 0.023 & 0.037 & 0.103 & 0.026 & 0.126 & 0.227 & 0.016 & 0.056 & 0.167 \\
\hline 124 & shu-003 & 0.003 & 0.006 & 0.013 & 0.002 & 0.004 & 0.008 & 0.003 & 0.005 & 0.009 & 0.008 & 0.031 & 0.070 & 0.004 & 0.014 & 0.040 \\
\hline 125 & sjtu-002 & 0.011 & 0.031 & 0.080 & 0.009 & 0.026 & 0.083 & 0.023 & 0.037 & 0.103 & 0.026 & 0.126 & 0.227 & 0.016 & 0.056 & 0.167 \\
\hline 126 & sjtu-003 & 0.002 & 0.004 & 0.009 & 0.002 & 0.003 & 0.006 & 0.002 & 0.003 & 0.006 & 0.005 & 0.022 & 0.049 & 0.003 & 0.009 & 0.028 \\
\hline 127 & staqu- 000 & 0.000 & 0.000 & 0.000 & 0.000 & 0.000 & 0.000 & 0.000 & 0.000 & 0.000 & 0.000 & 0.000 & 0.000 & 0.000 & 0.000 & 0.000 \\
\hline 128 & starhybrid-001 & 0.192 & 0.468 & 0.461 & 0.149 & 0.406 & 0.483 & 0.137 & 0.321 & 0.487 & 0.478 & 0.761 & 0.781 & 0.133 & 0.372 & 0.565 \\
\hline 129 & synesis-006 & 0.001 & 0.003 & 0.007 & 0.001 & 0.003 & 0.007 & 0.001 & 0.001 & 0.003 & 0.001 & 0.005 & 0.011 & 0.001 & 0.004 & 0.008 \\
\hline 130 & synesis-007 & 0.007 & 0.021 & 0.047 & 0.008 & 0.028 & 0.056 & 0.013 & 0.025 & 0.056 & 0.007 & 0.046 & 0.114 & 0.018 & 0.124 & 0.134 \\
\hline 131 & synology-000 & 0.000 & 0.000 & 0.000 & 0.000 & 0.000 & 0.000 & 0.000 & 0.000 & 0.000 & 0.000 & 0.000 & 0.000 & 0.000 & 0.000 & 0.000 \\
\hline 132 & synology-002 & 0.000 & 0.000 & 0.000 & 0.000 & 0.000 & 0.000 & 0.000 & 0.000 & 0.000 & 0.000 & 0.000 & 0.000 & 0.000 & 0.000 & 0.000 \\
\hline 133 & tech5-004 & 0.005 & 0.022 & 0.061 & 0.006 & 0.028 & 0.070 & 0.010 & 0.021 & 0.046 & 0.006 & 0.025 & 0.068 & 0.006 & 0.021 & 0.058 \\
\hline 134 & tech5-005 & 0.004 & 0.015 & 0.044 & 0.004 & 0.017 & 0.046 & 0.006 & 0.012 & 0.026 & 0.005 & 0.020 & 0.051 & 0.007 & 0.022 & 0.057 \\
\hline 135 & tevian-005 & 0.125 & 0.463 & 0.370 & 0.148 & 0.650 & 0.557 & 0.208 & 0.359 & 0.705 & 0.086 & 0.667 & 0.679 & 0.131 & 0.786 & 0.787 \\
\hline
\end{tabular}

Table 11: This table summarizes Failure to Enroll (FTE) rates surveyed over 10000 images of each mask variant. FTE is the proportion of failed template generation attempts. Failures can occur because the software throws an exception, or because the software electively refuses to process the input image as would occur if the algorithms does not detect a face or determines that the face has insufficient information. FTE is measured as the number of function calls that give EITHER a non-zero error code OR that give a "small" template containing fewer than 60 bytes. This second rule is needed because some algorithms incorrectly fail to return a non-zero error code when template generation fails but do produce a skeletal template. The effects of FTE are included in the accuracy results of this report by regarding any template comparison involving a failed template to produce a low similarity score. Thus higher FTE results in higher FNMR and lower FMR. Algorithms in black were submitted prior to mid-March 2020, and algorithms in blue were submitted thereafter. 


\begin{tabular}{|c|c|c|c|c|c|c|c|c|c|c|c|c|c|c|c|c|}
\hline & \multirow{3}{*}{$\begin{array}{l}\text { Algorithm } \\
\text { Name } \\
\text { COVERAGE } \\
\end{array}$} & \multirow{2}{*}{\multicolumn{3}{|c|}{$\begin{array}{l}\text { COLOR }=\text { WHITE } \\
\text { SHAPE }=\text { WIDE }\end{array}$}} & \multicolumn{6}{|c|}{ COLOR = LIGHTBLUE } & \multirow{2}{*}{\multicolumn{3}{|c|}{$\begin{array}{l}\text { COLOR }=\text { RED } \\
\text { SHAPE }=\text { WIDE }\end{array}$}} & \multirow{2}{*}{\multicolumn{3}{|c|}{$\begin{array}{l}\text { COLOR }=\text { BLACK } \\
\text { SHAPE }=\text { WIDE }\end{array}$}} \\
\hline & & & & & \multicolumn{3}{|c|}{ SHAPE $=$ WIDE } & \multicolumn{3}{|c|}{ SHAPE $=$ ROUND } & & & & & & \\
\hline & & $\mathrm{LO}$ & MED & $\mathrm{HI}$ & $\mathrm{LO}$ & MED & $\mathrm{HI}$ & $\mathrm{LO}$ & MED & $\mathrm{HI}$ & $\mathrm{LO}$ & MED & $\mathrm{HI}$ & $\mathrm{LO}$ & MED & $\mathrm{HI}$ \\
\hline 136 & tevian-006 & 0.012 & 0.089 & 0.160 & 0.010 & 0.066 & 0.124 & 0.028 & 0.041 & 0.146 & 0.034 & 0.266 & 0.408 & 0.016 & 0.256 & 0.307 \\
\hline 137 & trueface- 000 & 0.000 & 0.000 & 0.000 & 0.000 & 0.000 & 0.000 & 0.000 & 0.000 & 0.000 & 0.000 & 0.000 & 0.000 & 0.000 & 0.000 & 0.000 \\
\hline 138 & trueface-001 & 0.078 & 0.163 & 0.217 & 0.071 & 0.153 & 0.217 & 0.084 & 0.119 & 0.268 & & & & 0.147 & 0.317 & 0.474 \\
\hline 139 & tuputech-000 & 0.517 & 0.679 & 0.684 & 0.626 & 0.758 & 0.765 & 0.502 & 0.619 & 0.714 & 0.652 & 0.893 & 0.926 & 0.661 & 0.904 & 0.933 \\
\hline 140 & uluface- 002 & 0.000 & 0.000 & 0.000 & 0.000 & 0.000 & 0.000 & 0.000 & 0.000 & 0.000 & 0.000 & 0.000 & 0.000 & 0.000 & 0.000 & 0.000 \\
\hline$\overline{141}$ & upc-001 & 0.002 & 0.005 & 0.012 & 0.002 & 0.005 & $\overline{0.012}$ & 0.002 & 0.002 & 0.005 & 0.002 & 0.010 & 0.027 & 0.003 & 0.007 & 0.018 \\
\hline 142 & veridas- 003 & 1.000 & 1.000 & 1.000 & 1.000 & 1.000 & 1.000 & 1.000 & 1.000 & 1.000 & 1.000 & 1.000 & 1.000 & 1.000 & 1.000 & 1.000 \\
\hline 143 & veridas-004 & 0.047 & 0.139 & 0.202 & 0.041 & 0.112 & 0.164 & 0.048 & 0.085 & 0.154 & 0.106 & 0.401 & 0.568 & 0.054 & 0.213 & 0.383 \\
\hline 144 & via-001 & 0.000 & 0.000 & 0.000 & 0.000 & 0.000 & 0.000 & 0.000 & 0.000 & 0.000 & 0.000 & 0.000 & 0.000 & 0.000 & 0.000 & 0.000 \\
\hline 145 & videmo-000 & 0.019 & 0.067 & 0.125 & 0.018 & 0.051 & 0.106 & 0.023 & 0.040 & 0.089 & 0.122 & 0.352 & 0.368 & 0.027 & 0.100 & 0.296 \\
\hline 146 & videonetics-002 & 0.338 & 0.581 & 0.557 & 0.330 & 0.569 & 0.542 & 0.378 & 0.559 & 0.785 & 0.344 & 0.693 & 0.735 & 0.396 & 0.702 & 0.848 \\
\hline 147 & vigilantsolutions-007 & 0.062 & 0.168 & 0.220 & 0.052 & 0.137 & 0.193 & 0.069 & 0.126 & 0.206 & 0.098 & 0.408 & 0.634 & 0.072 & 0.273 & 0.493 \\
\hline 148 & vigilantsolutions-008 & 0.062 & 0.168 & 0.218 & 0.051 & 0.136 & 0.189 & 0.068 & 0.124 & 0.202 & 0.096 & 0.407 & 0.633 & 0.072 & 0.268 & 0.492 \\
\hline 149 & vinai-000 & 0.000 & 0.000 & 0.000 & 0.000 & 0.000 & 0.000 & 0.000 & 0.000 & 0.000 & 0.000 & 0.000 & 0.000 & 0.000 & 0.000 & 0.000 \\
\hline 150 & visionlabs-008 & 0.013 & 0.035 & 0.083 & 0.012 & 0.031 & 0.072 & 0.019 & 0.038 & 0.097 & 0.016 & 0.040 & 0.096 & 0.024 & 0.061 & 0.124 \\
\hline$\overline{151}$ & "visionlabs-009 & 0.011 & 0.037 & $\overline{0.082}$ & $\overline{0.010}$ & 0.035 & 0.076 & $\overline{0.018}$ & $\overline{0.042}$ & 0.097 & $\overline{0.016}$ & 0.052 & 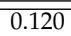 & 0.024 & 0.065 & $\overline{0.150}$ \\
\hline 152 & visteam-000 & 0.058 & 0.150 & 0.210 & 0.048 & 0.118 & 0.176 & 0.052 & 0.092 & 0.156 & 0.160 & 0.372 & 0.506 & 0.074 & 0.202 & 0.369 \\
\hline 153 & vocord-008 & 0.013 & 0.046 & 0.087 & 0.011 & 0.052 & 0.089 & 0.031 & 0.059 & 0.111 & 0.013 & 0.079 & 0.127 & 0.009 & 0.050 & 0.093 \\
\hline 154 & vts-000 & 0.011 & 0.029 & 0.072 & 0.012 & 0.028 & 0.074 & 0.015 & 0.020 & 0.048 & 0.014 & 0.043 & 0.129 & 0.016 & 0.051 & 0.149 \\
\hline 155 & winsense-001 & 0.000 & 0.000 & 0.000 & 0.000 & 0.000 & 0.000 & 0.000 & 0.000 & 0.000 & 0.000 & 0.000 & 0.000 & 0.000 & 0.000 & 0.000 \\
\hline 156 & xforwardai- 000 & 0.000 & 0.000 & 0.000 & 0.000 & 0.000 & 0.000 & 0.000 & 0.000 & 0.000 & 0.000 & 0.000 & 0.000 & 0.000 & 0.000 & 0.000 \\
\hline 157 & xforwardai-001 & 0.006 & 0.025 & 0.069 & 0.007 & 0.031 & 0.079 & 0.011 & 0.021 & 0.052 & 0.007 & 0.030 & 0.079 & 0.007 & 0.022 & 0.060 \\
\hline 158 & $x \mathrm{xm}-000$ & 0.003 & 0.006 & 0.013 & 0.002 & 0.004 & 0.008 & 0.003 & 0.004 & 0.009 & 0.008 & 0.031 & 0.070 & 0.005 & 0.013 & 0.038 \\
\hline 159 & yoonik-000 & 0.029 & 0.061 & 0.138 & 0.027 & 0.060 & 0.142 & 0.037 & 0.049 & 0.124 & 0.040 & 0.094 & 0.219 & 0.039 & 0.099 & 0.257 \\
\hline 160 & yoonik-001 & 0.015 & 0.052 & 0.110 & 0.017 & 0.055 & 0.113 & 0.024 & 0.035 & 0.072 & 0.031 & 0.093 & 0.170 & 0.026 & 0.086 & 0.175 \\
\hline$\overline{161}$ & yuan-000 & $\overline{0.000}$ & 0.000 & $\overline{0.000}$ & 0.000 & $\overline{0.000}$ & $\overline{0.000}$ & $\overline{0.000}$ & $\overline{0.000}$ & $\overline{0.000}$ & $\overline{0.000}$ & $\overline{0.000}$ & $\overline{0.000}$ & 0.000 & $\overline{0.000}$ & $\overline{0.000}$ \\
\hline
\end{tabular}

Table 12: This table summarizes Failure to Enroll (FTE) rates surveyed over 10000 images of each mask variant. FTE is the proportion of failed template generation attempts. Failures can occur because the software throws an exception, or because the software electively refuses to process the input image as would occur if the algorithms does not detect a face or determines that the face has insufficient information. FTE is measured as the number of function calls that give EITHER a non-zero error code OR that give a "small" template containing fewer than 60 bytes. This second rule is needed because some algorithms incorrectly fail to return a non-zero error code when template generation fails but do produce a skeletal template. The effects of FTE are included in the accuracy results of this report by regarding any template comparison involving a failed template to produce a low similarity score. Thus higher FTE results in higher FNMR and lower FMR. Algorithms in black were submitted prior to mid-March 2020, and algorithms in blue were submitted thereafter. 
Failure-to-template contribution toward total false rejection for medium wide lightblue masks

Kind $\bullet$ FNMR $\bullet$ FTE

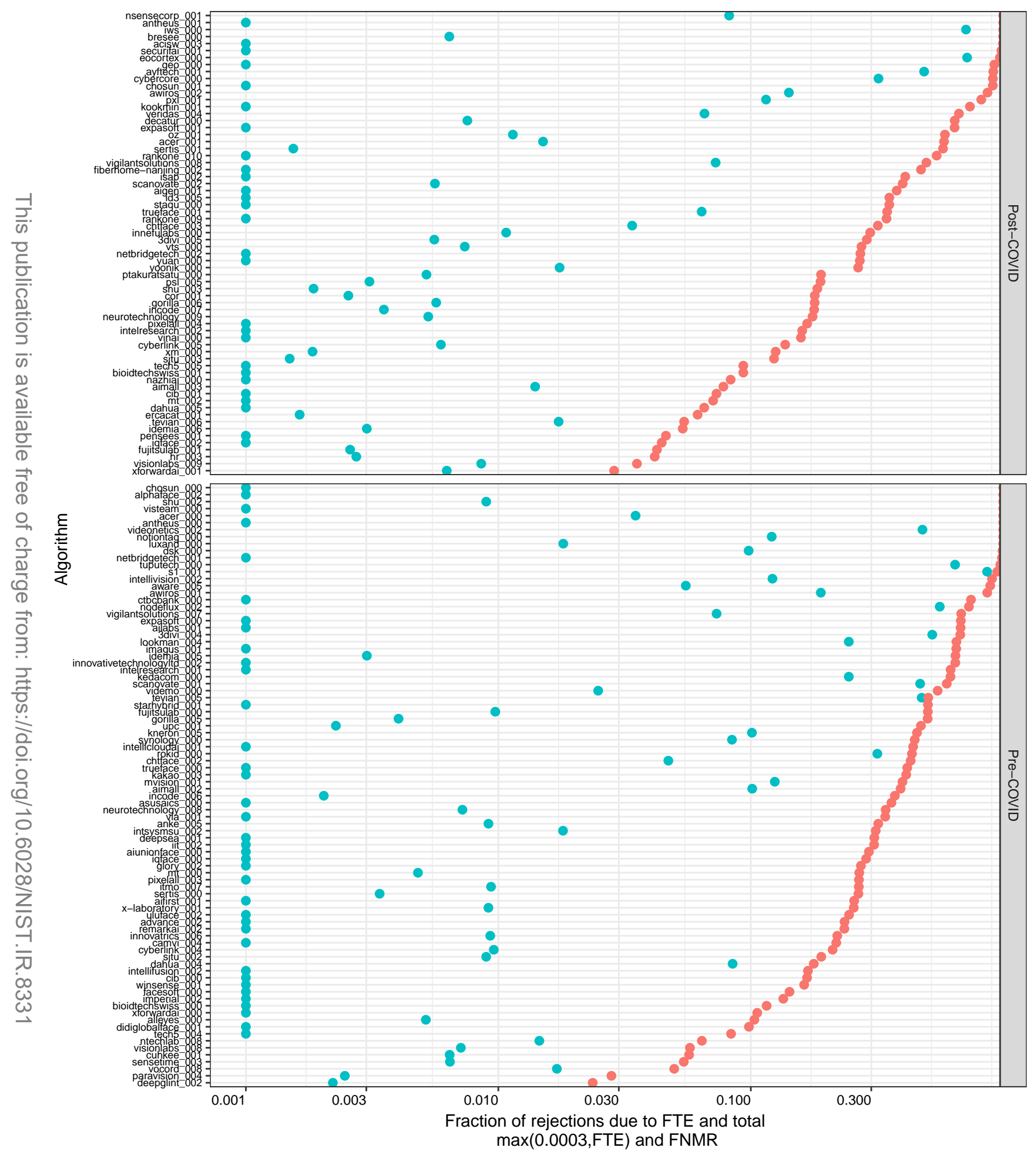

Figure 10: For each algorithm the rightmost dot shows FNMR @ FMR=0.00001 (as reported throughout this report). The left most dot shows the failure-to-template (FTE) rate over the masked verification set of 5.2M images. The gap between the two dots is attributable to low similarity score. Some FTE rates are zero - rates below 0.001 are shown as 0.001 . 
The following plots are detection error tradeoff (DET) characteristics for each algorithm, across different mask nose coverages and shapes. 

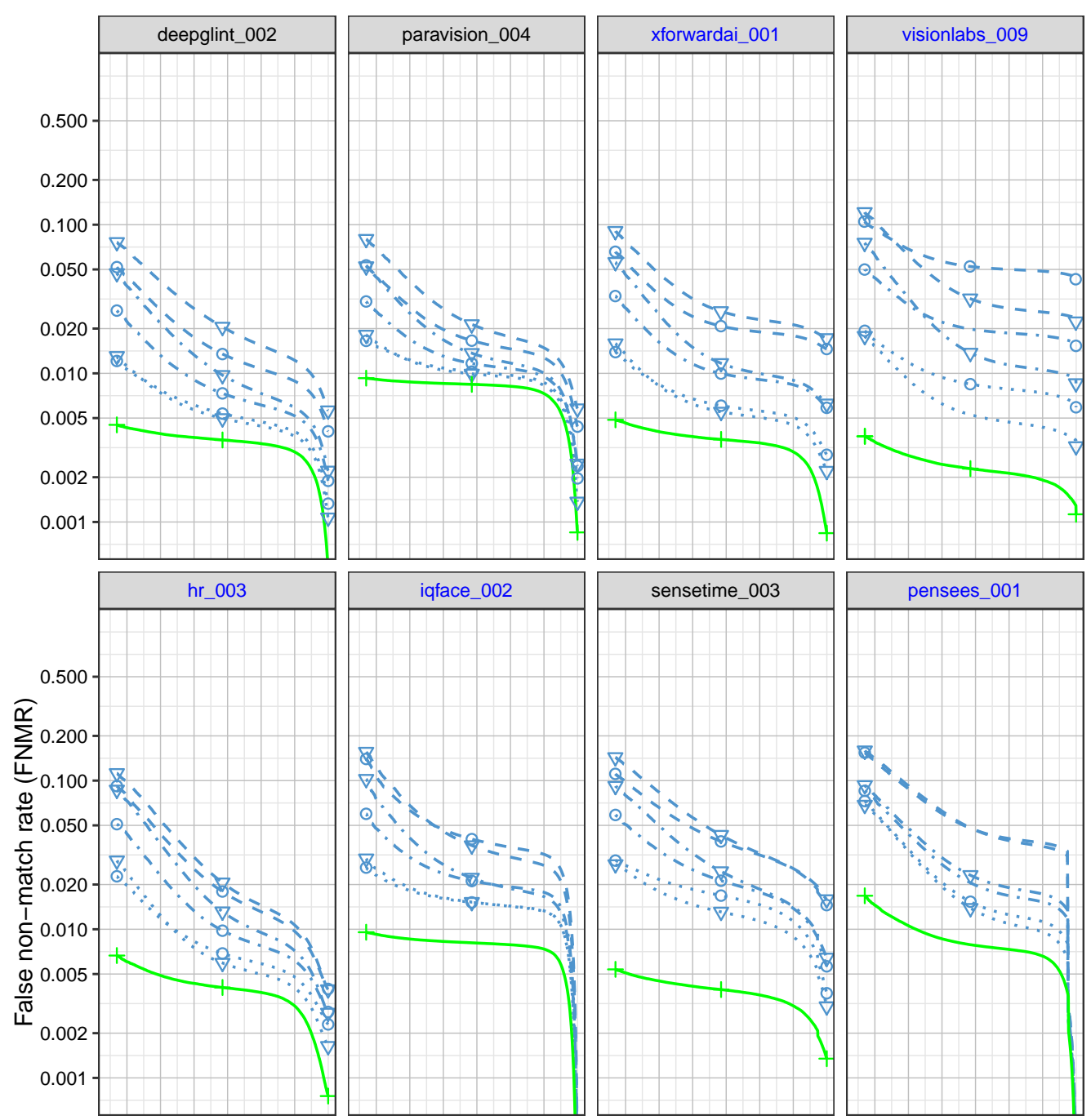

\section{Mask Nose Coverage}

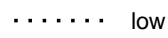

$\cdot-\cdot$ medium

- - - high

not masked

Mask Color

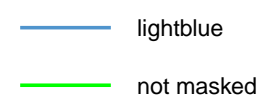

Mask Shape

$\begin{array}{ll}\nabla & \text { wide } \\ \circ & \text { round }\end{array}$
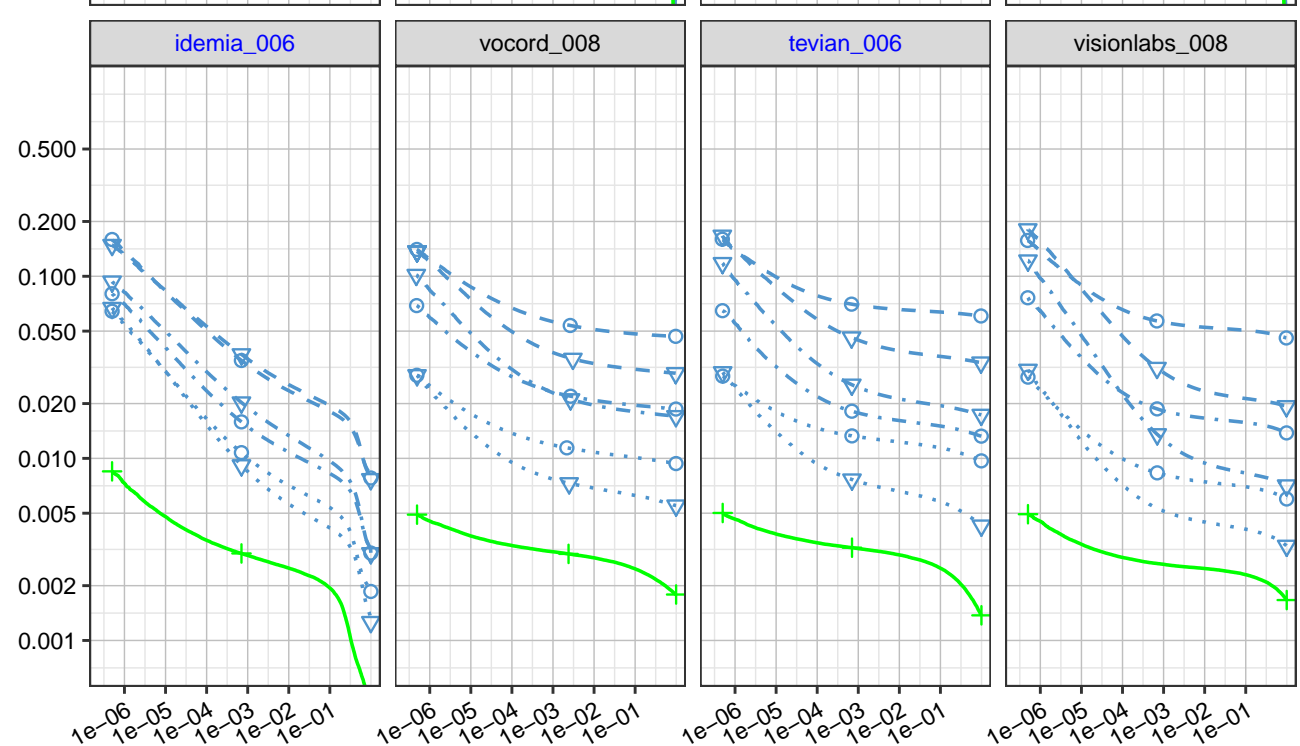

$+\quad$ not masked

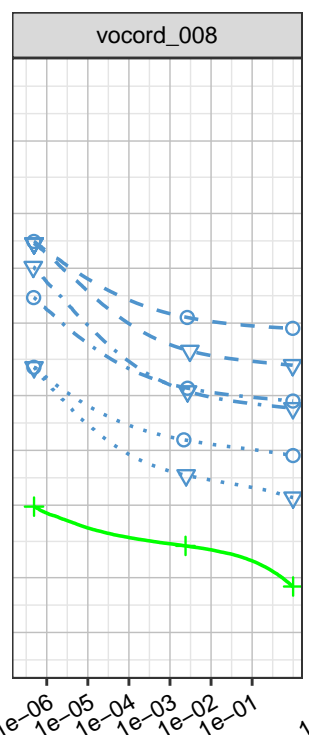

False match rate (FMR)

Figure 11: DET curves showing error rates on unmasked and masked probe images, broken out by mask shape and nose coverage. Algorithms in black were submitted prior to mid-March 2020, and algorithms in blue were submitted thereafter. 

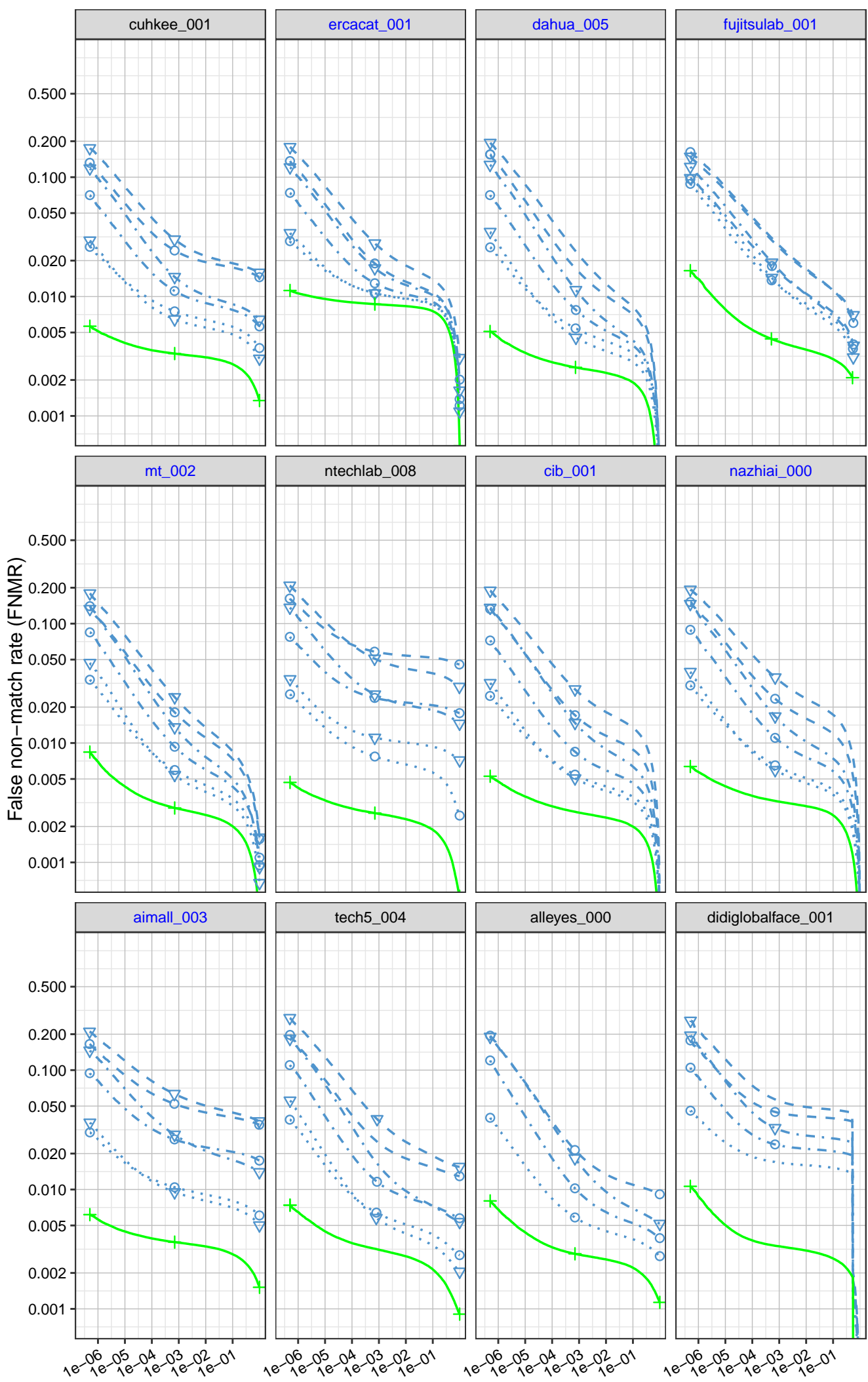

\section{Mask Nose Coverage}

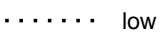

$\cdot-\cdot$ - medium

- - . high

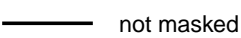

Mask Color

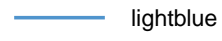

$\smile$ not masked

\section{Mask Shape}

\section{$\nabla \quad$ wide}

○ round
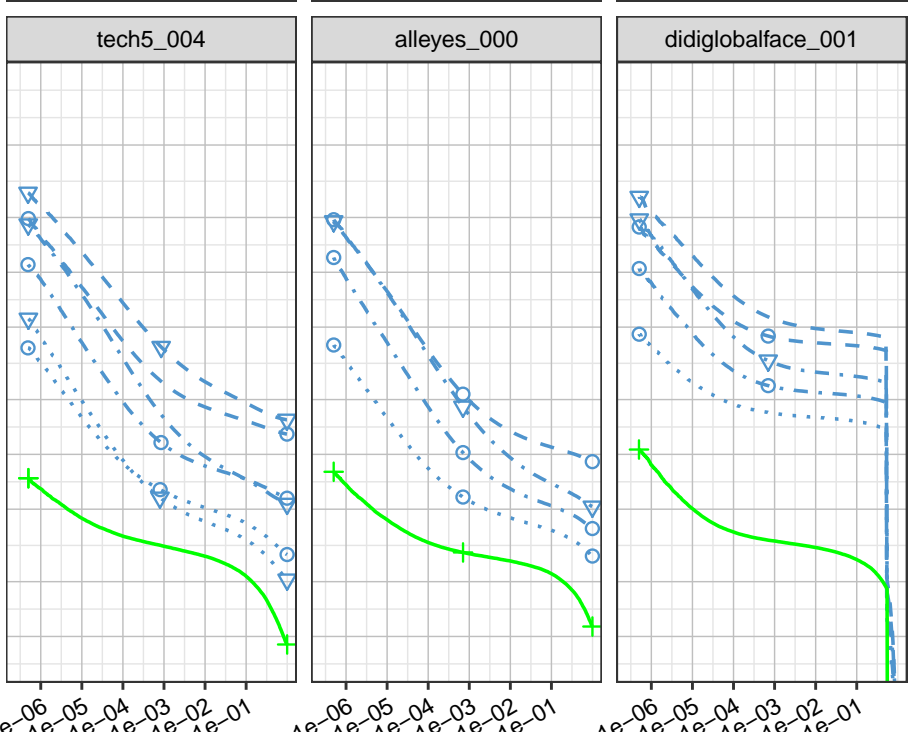

$+\quad$ not masked

False match rate (FMR)

Figure 12: DET curves showing error rates on unmasked and masked probe images, broken out by mask shape and nose coverage. Algorithms in black were submitted prior to mid-March 2020, and algorithms in blue were submitted thereafter. 

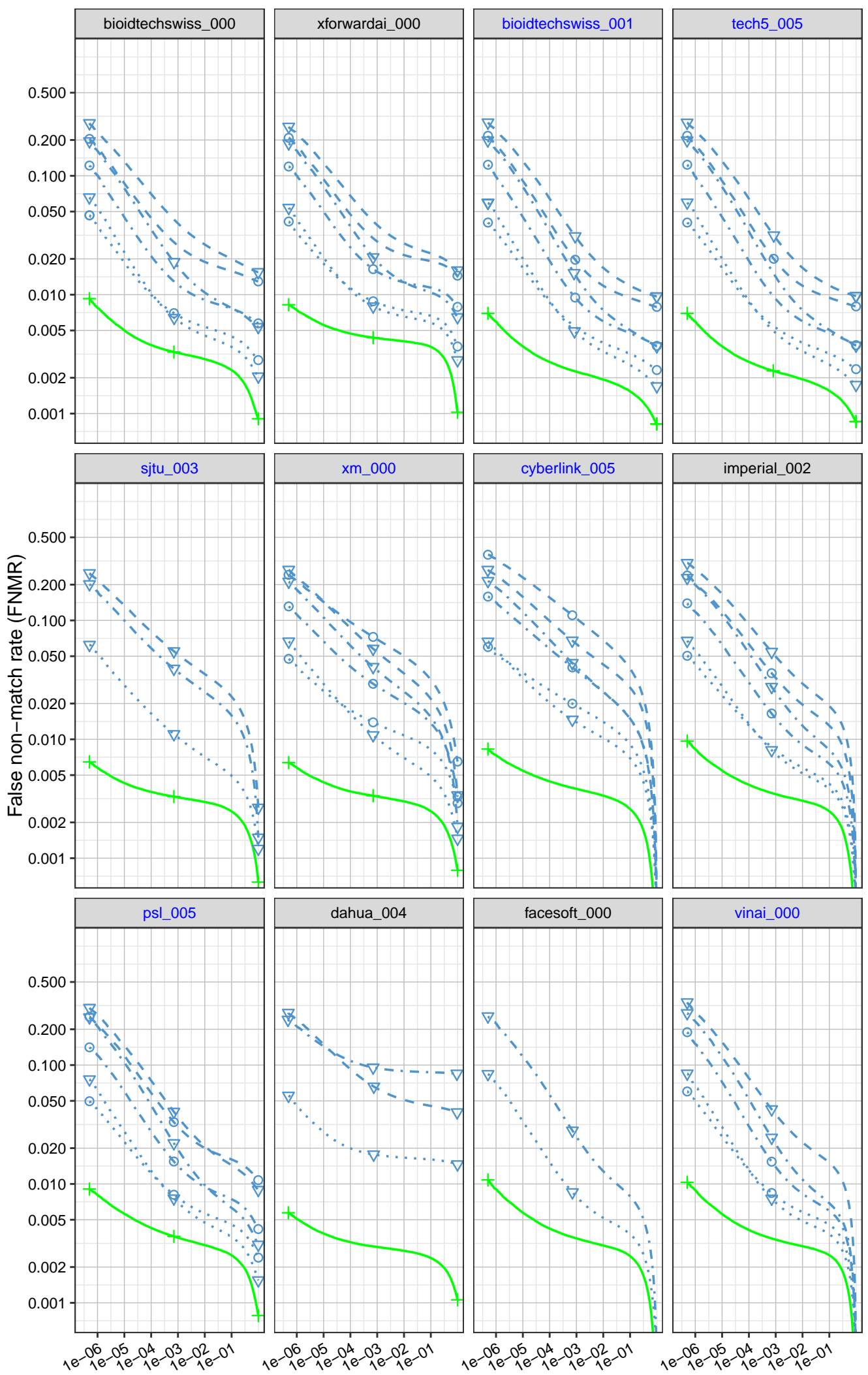

\section{Mask Nose Coverage}

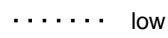

$\cdot-\cdot$ medium

- - . high

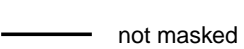

Mask Color

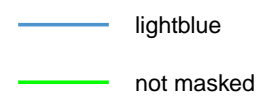

\section{Mask Shape}

\begin{tabular}{ll}
$\nabla$ & wide \\
\hline & round \\
+ & not masked
\end{tabular}
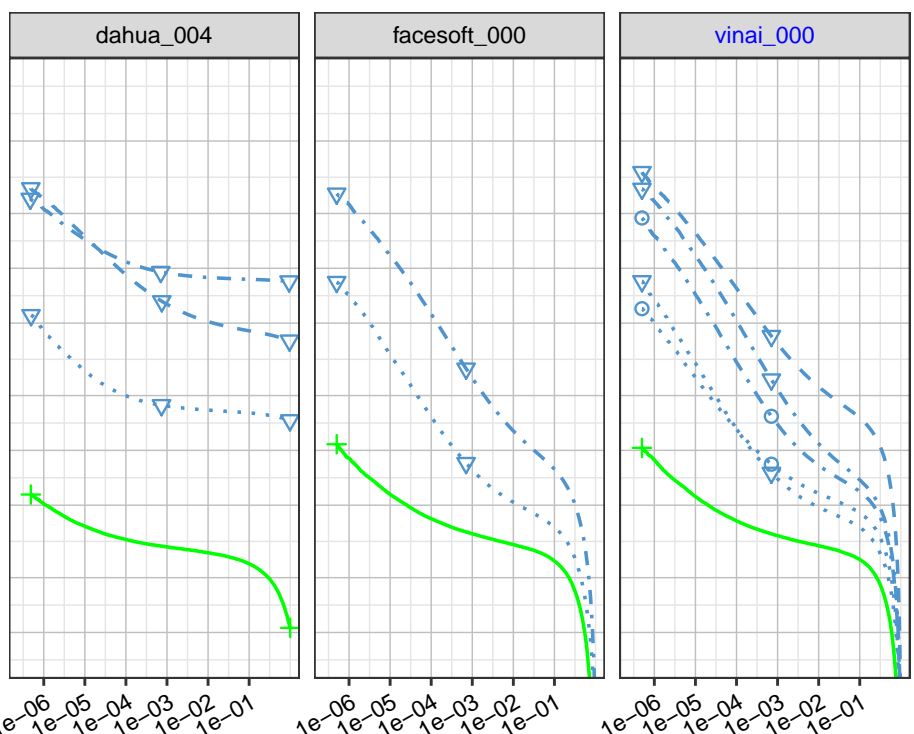

$+\quad$ not masked

False match rate (FMR)

Figure 13: DET curves showing error rates on unmasked and masked probe images, broken out by mask shape and nose coverage. Algorithms in black were submitted prior to mid-March 2020, and algorithms in blue were submitted thereafter. 

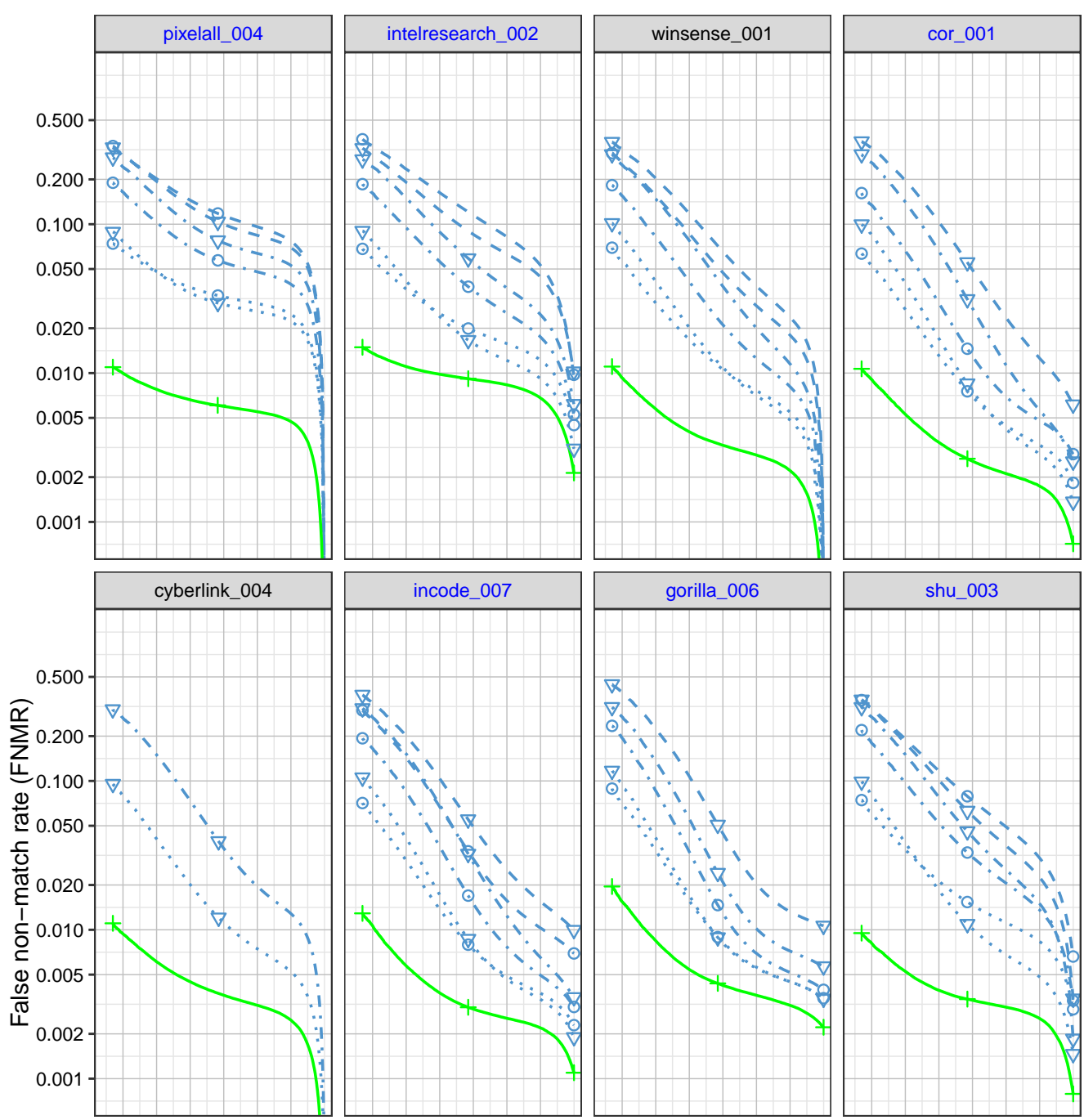

\section{Mask Nose Coverage}

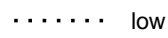

$\cdot-\cdot$ - medium

- - - high

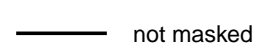

Mask Color

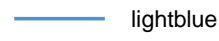

not masked

Mask Shape

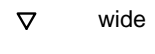

$\circ$ round

$+\quad$ not masked
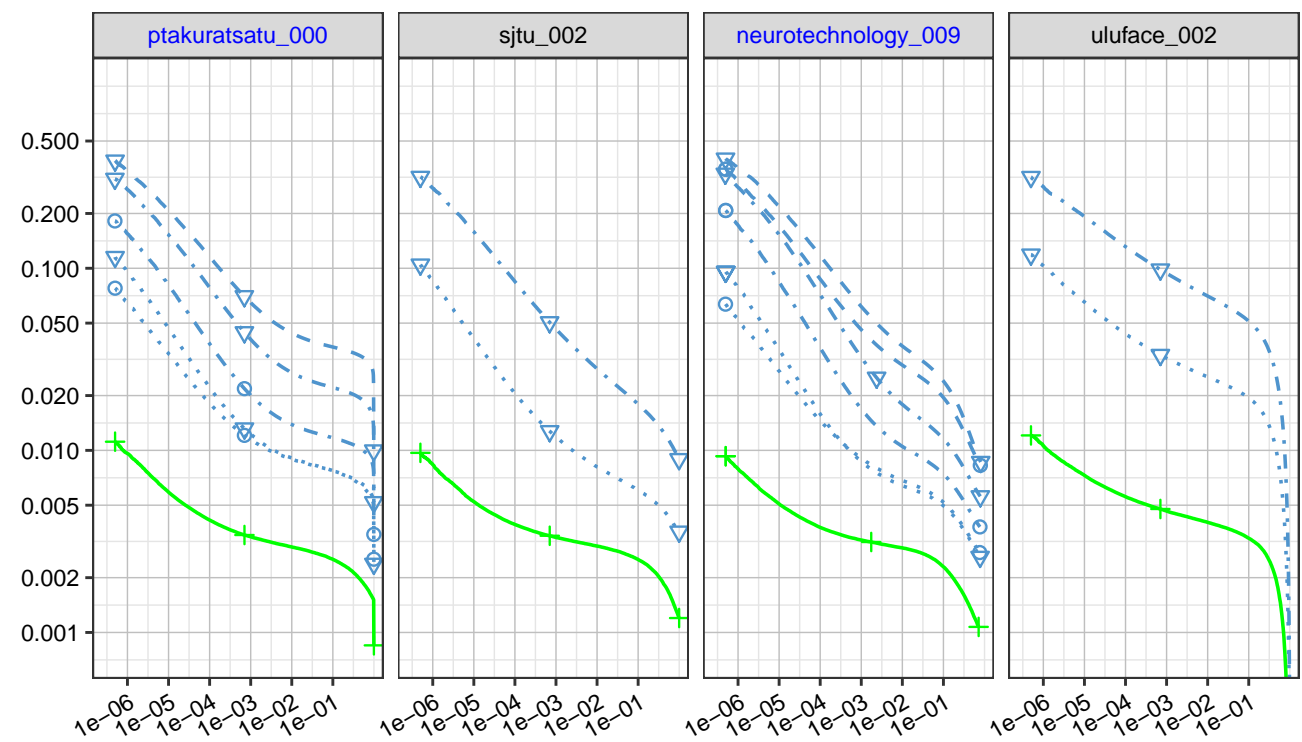

False match rate (FMR)

Figure 14: DET curves showing error rates on unmasked and masked probe images, broken out by mask shape and nose coverage. Algorithms in black were submitted prior to mid-March 2020, and algorithms in blue were submitted thereafter. 

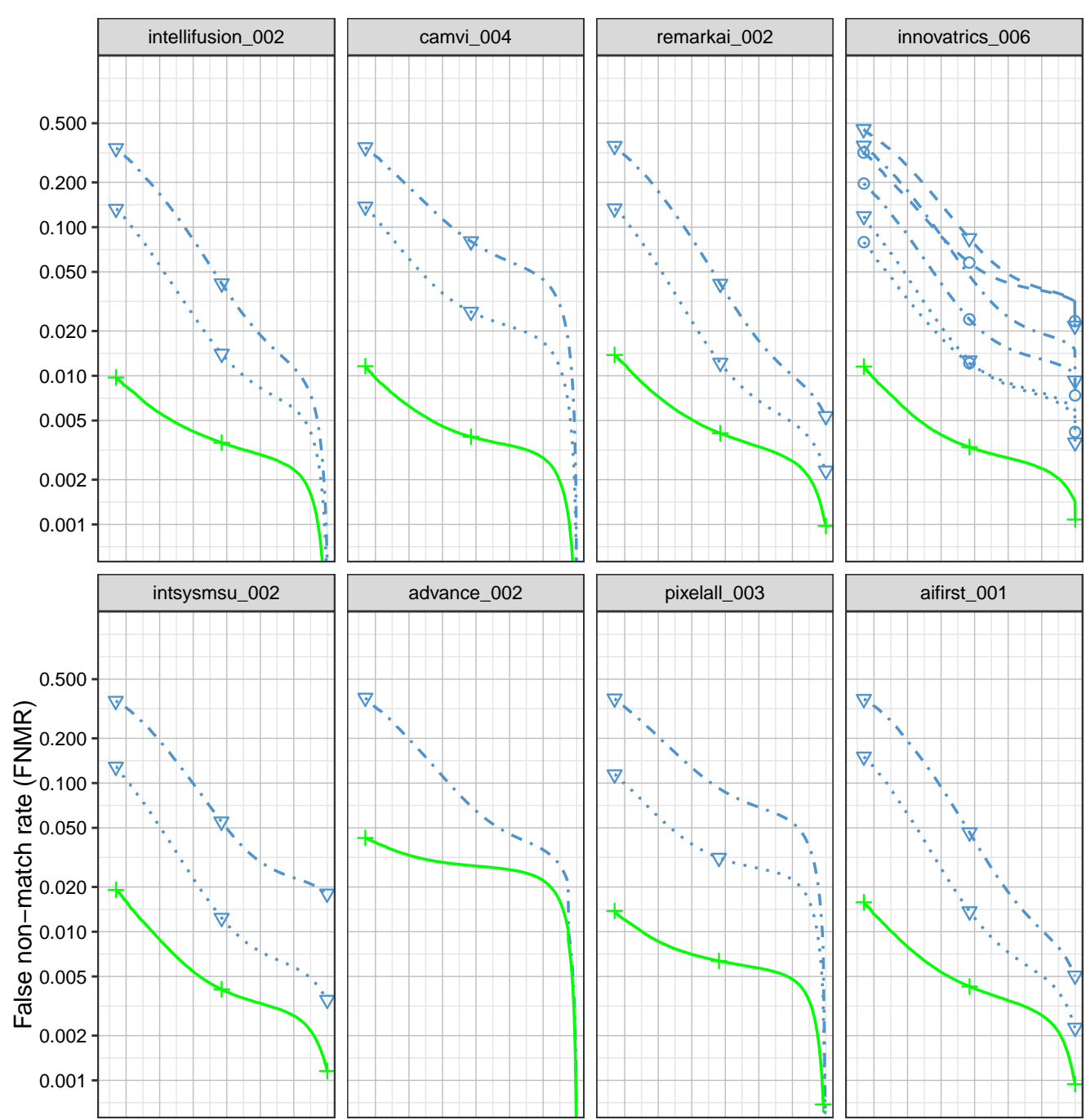

\section{Mask Nose Coverage}

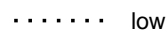

$\cdot-\cdot-\cdot$ medium

- - . high

not masked

Mask Color

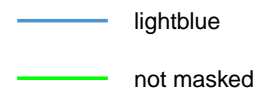

Mask Shape

$\nabla \quad$ wide

o round
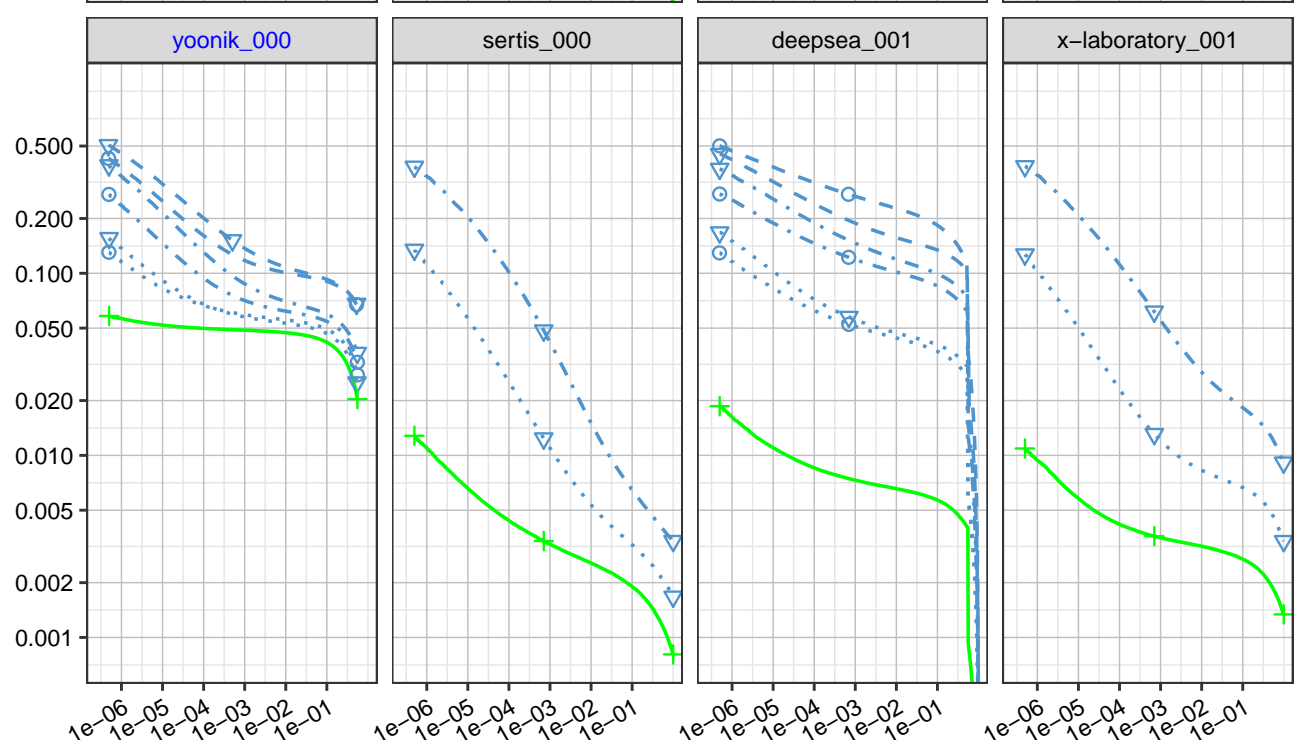

$+\quad$ not masked

False match rate (FMR)

Figure 15: DET curves showing error rates on unmasked and masked probe images, broken out by mask shape and nose coverage. Algorithms in black were submitted prior to mid-March 2020, and algorithms in blue were submitted thereafter. 

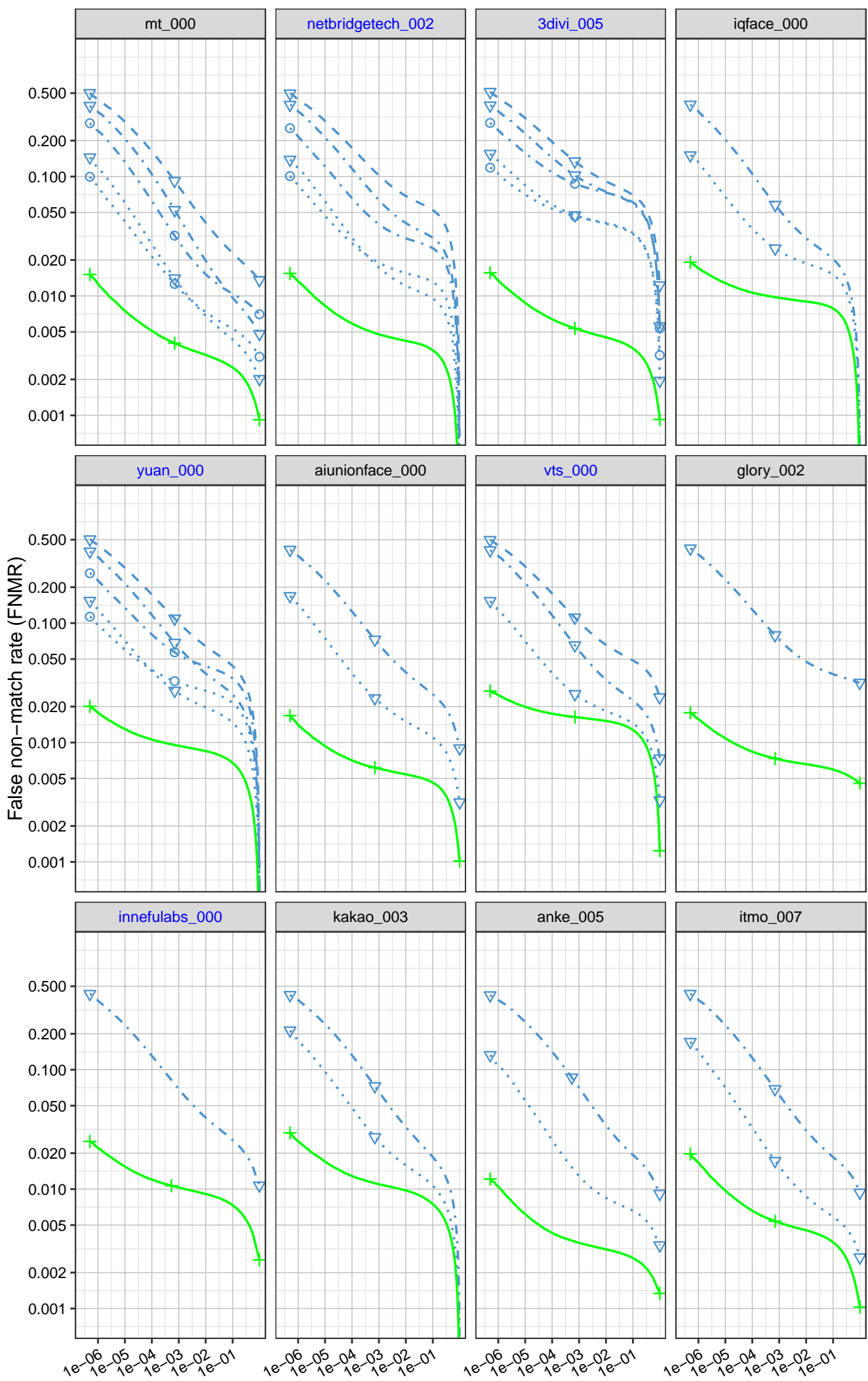

\section{Mask Nose Coverage}

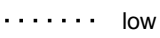

$\cdot-\cdot-\cdot$ medium

- - - high

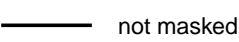

Mask Color

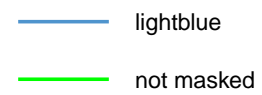

Mask Shape

$\nabla \quad$ wide

$\circ$ round
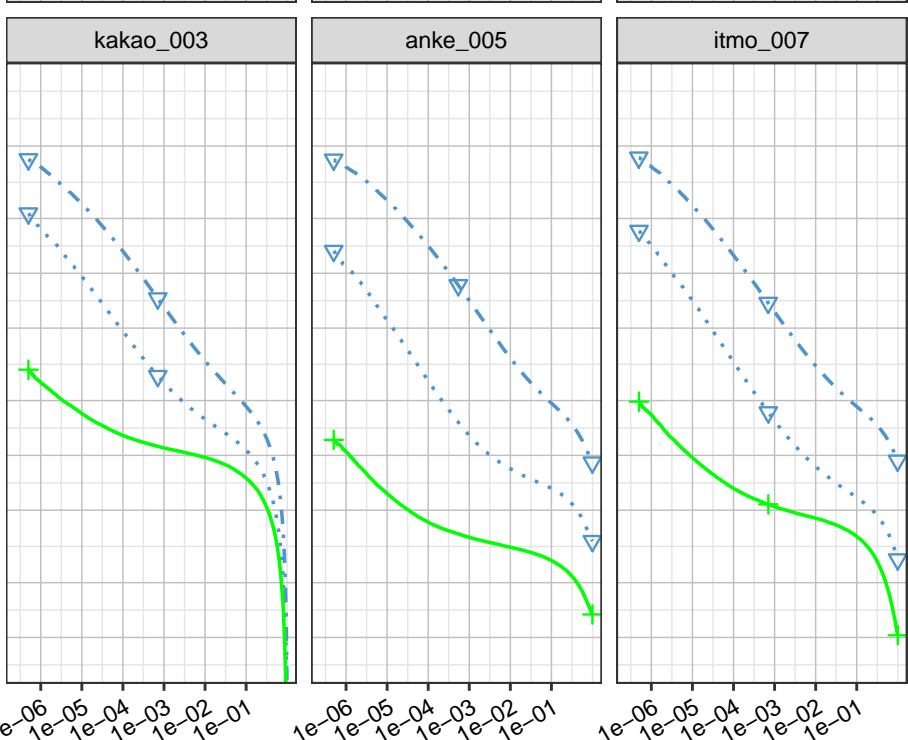

$+\quad$ not masked

False match rate (FMR)

Figure 16: DET curves showing error rates on unmasked and masked probe images, broken out by mask shape and nose coverage. Algorithms in black were submitted prior to mid-March 2020, and algorithms in blue were submitted thereafter. 

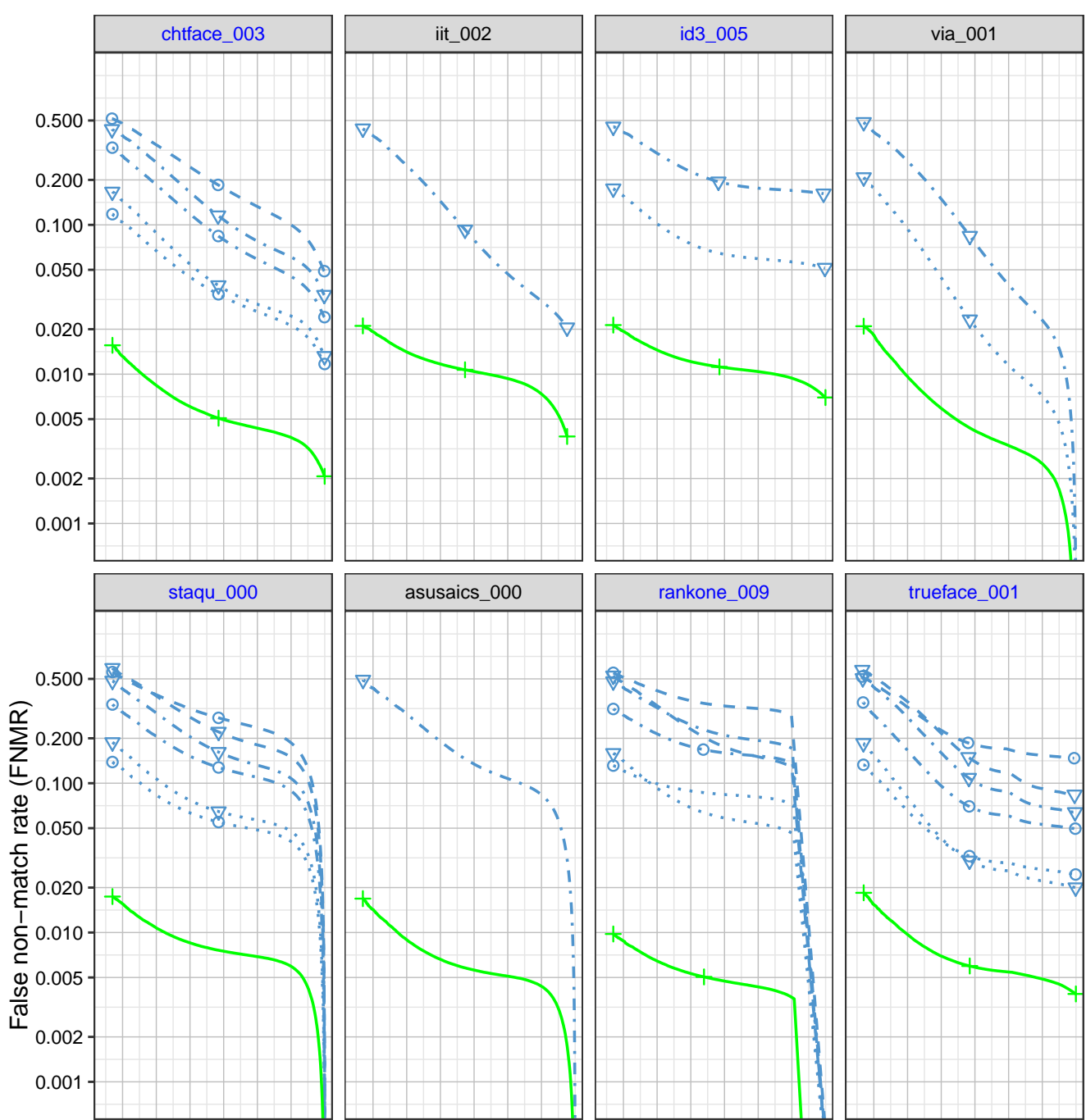

\section{Mask Nose Coverage}

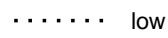

$\cdot-\cdot$ - medium

- - - high

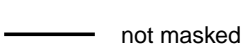

Mask Color
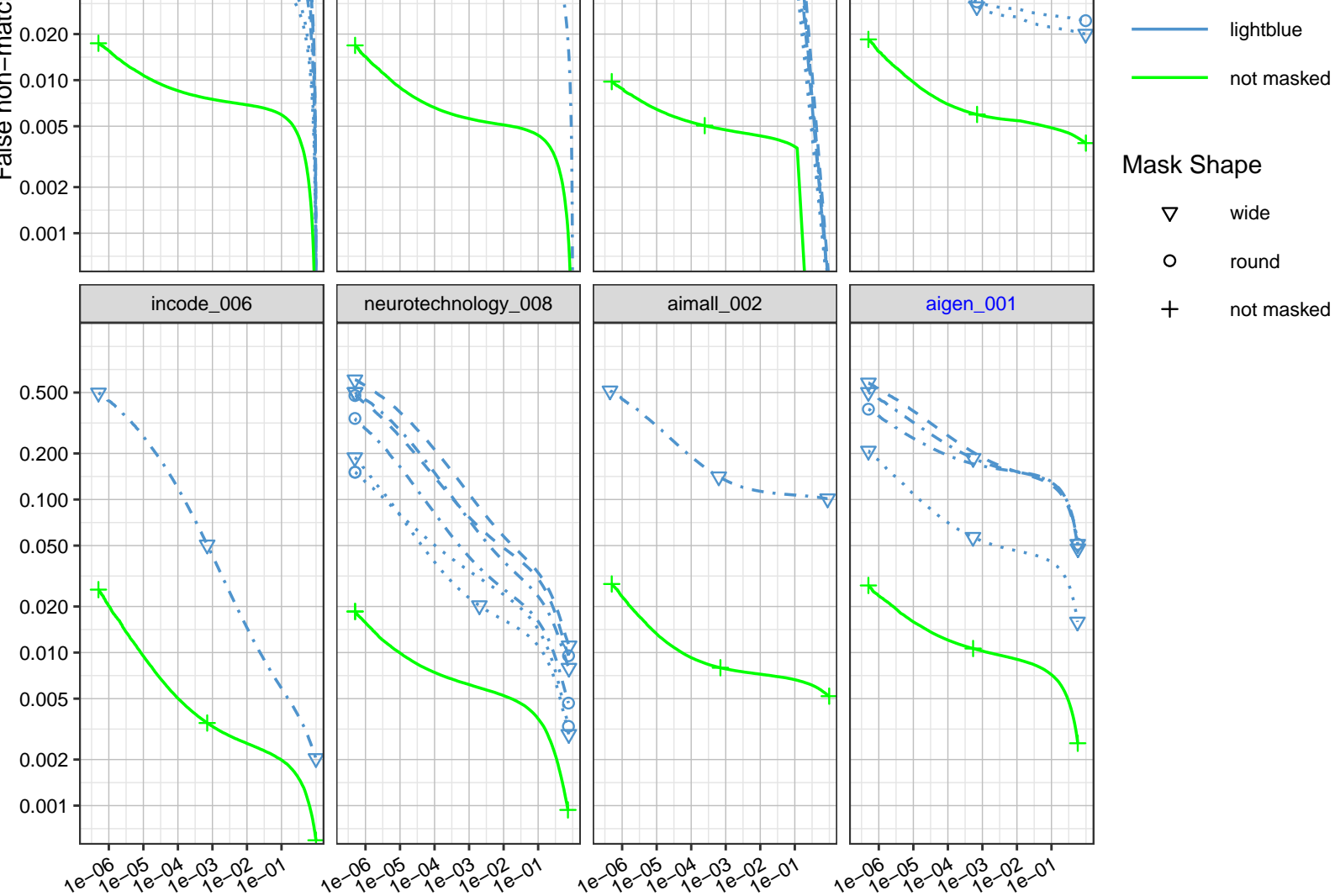

Mask Shape

$\begin{array}{ll}\nabla & \text { wide } \\ \circ & \text { round } \\ + & \text { not masked }\end{array}$

False match rate (FMR)

Figure 17: DET curves showing error rates on unmasked and masked probe images, broken out by mask shape and nose coverage. Algorithms in black were submitted prior to mid-March 2020, and algorithms in blue were submitted thereafter. 

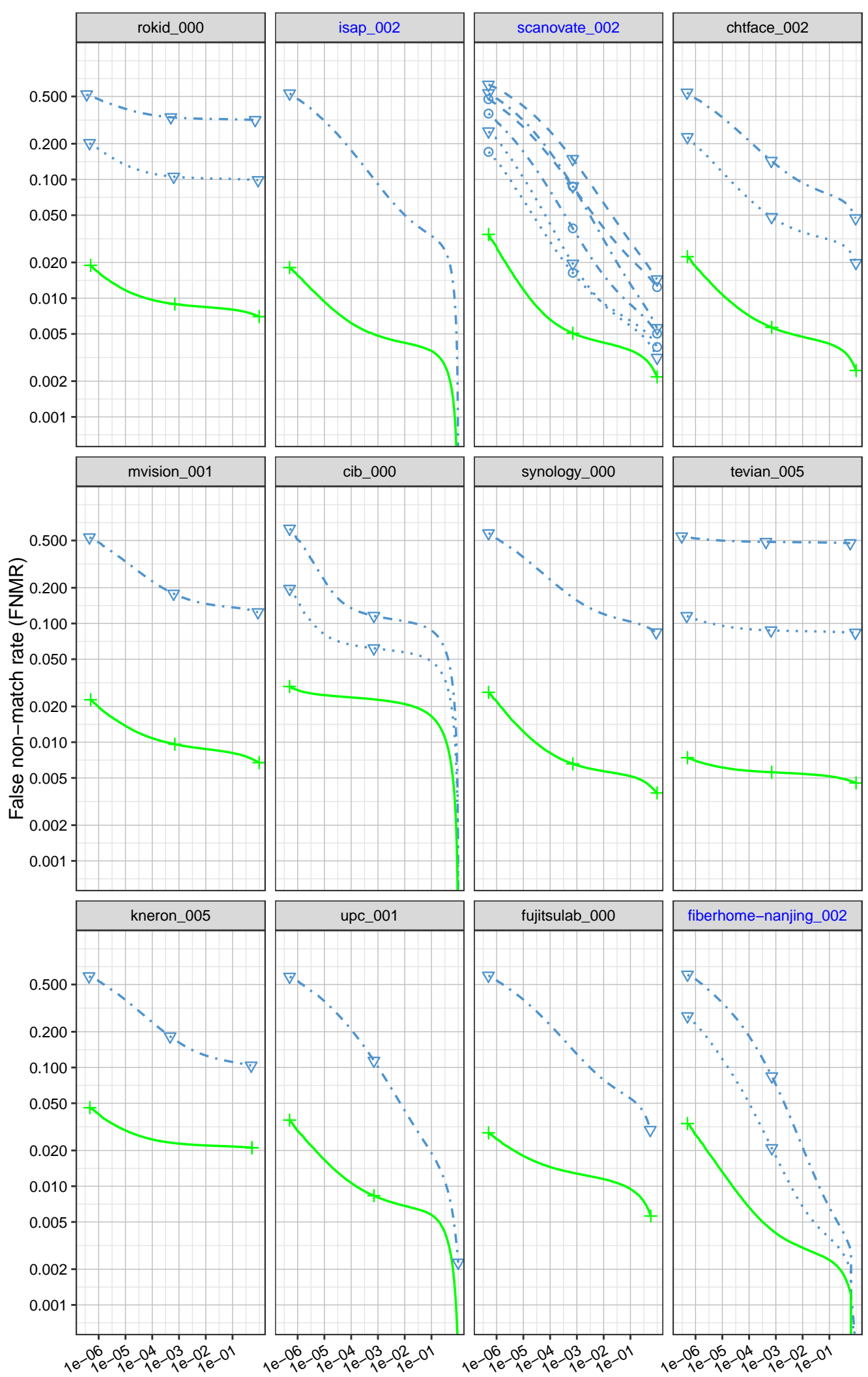

\section{Mask Nose Coverage}

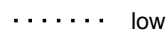

$\cdot-\cdot$ medium

- - - high

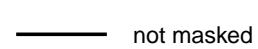

Mask Color

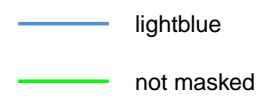

Mask Shape

$\nabla \quad$ wide

$\circ$ round
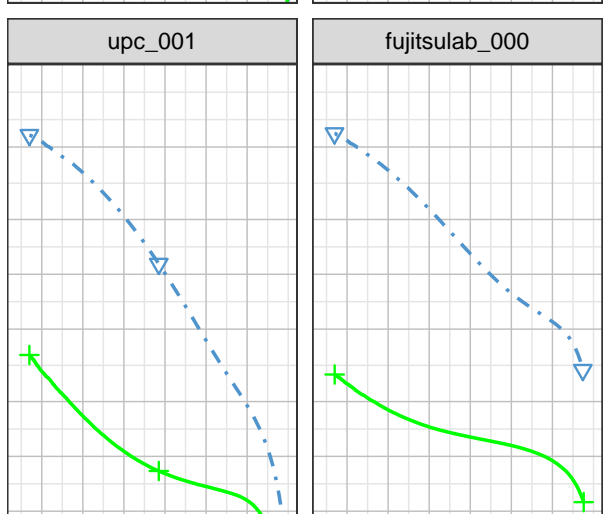

fiberhome-nanjing_002

$+\quad$ not masked

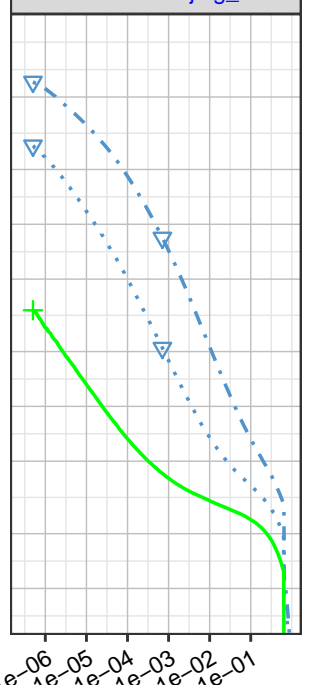

\section{False match rate (FMR)}

Figure 18: DET curves showing error rates on unmasked and masked probe images, broken out by mask shape and nose coverage. Algorithms in black were submitted prior to mid-March 2020, and algorithms in blue were submitted thereafter. 


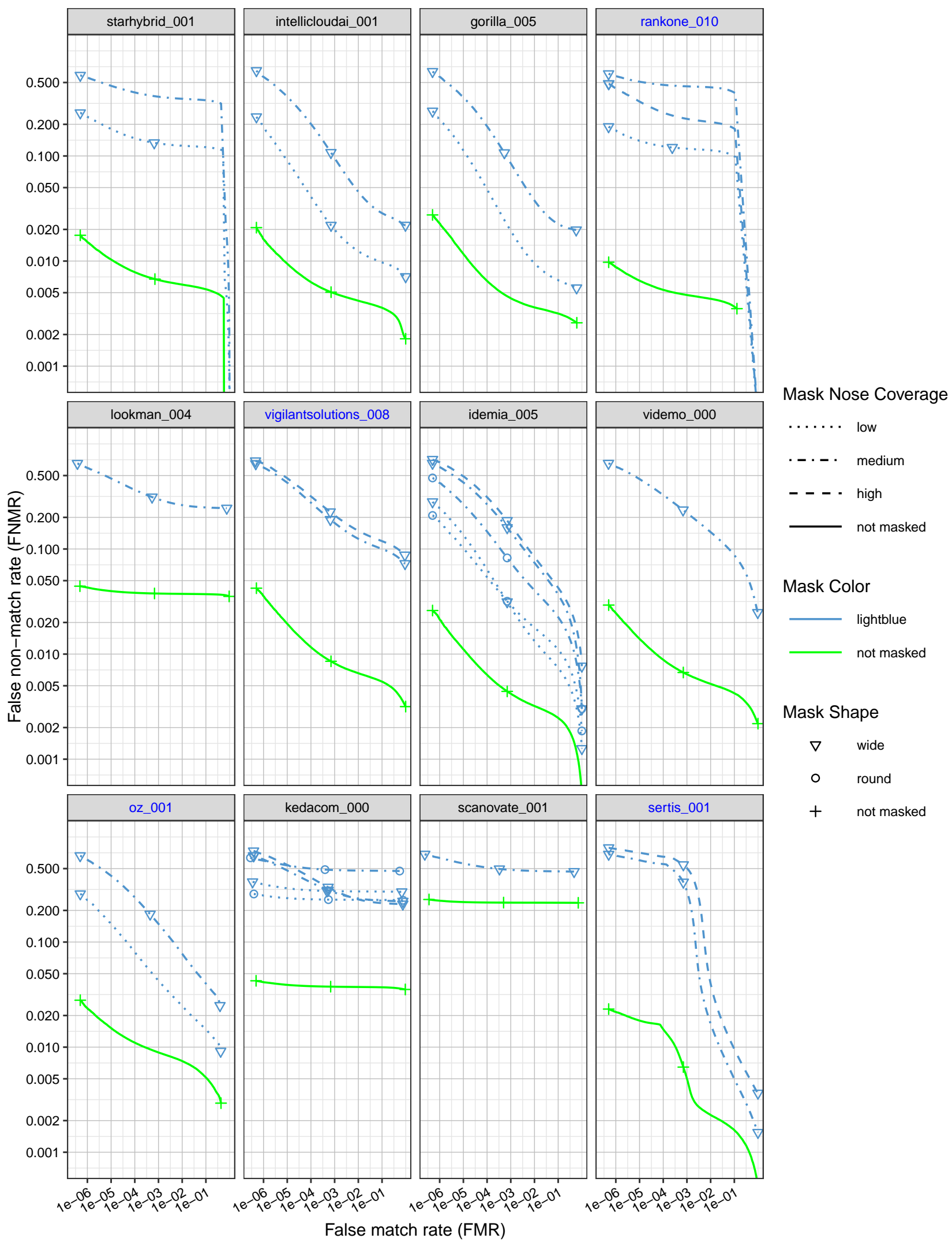

Figure 19: DET curves showing error rates on unmasked and masked probe images, broken out by mask shape and nose coverage. Algorithms in black were submitted prior to mid-March 2020, and algorithms in blue were submitted thereafter. 

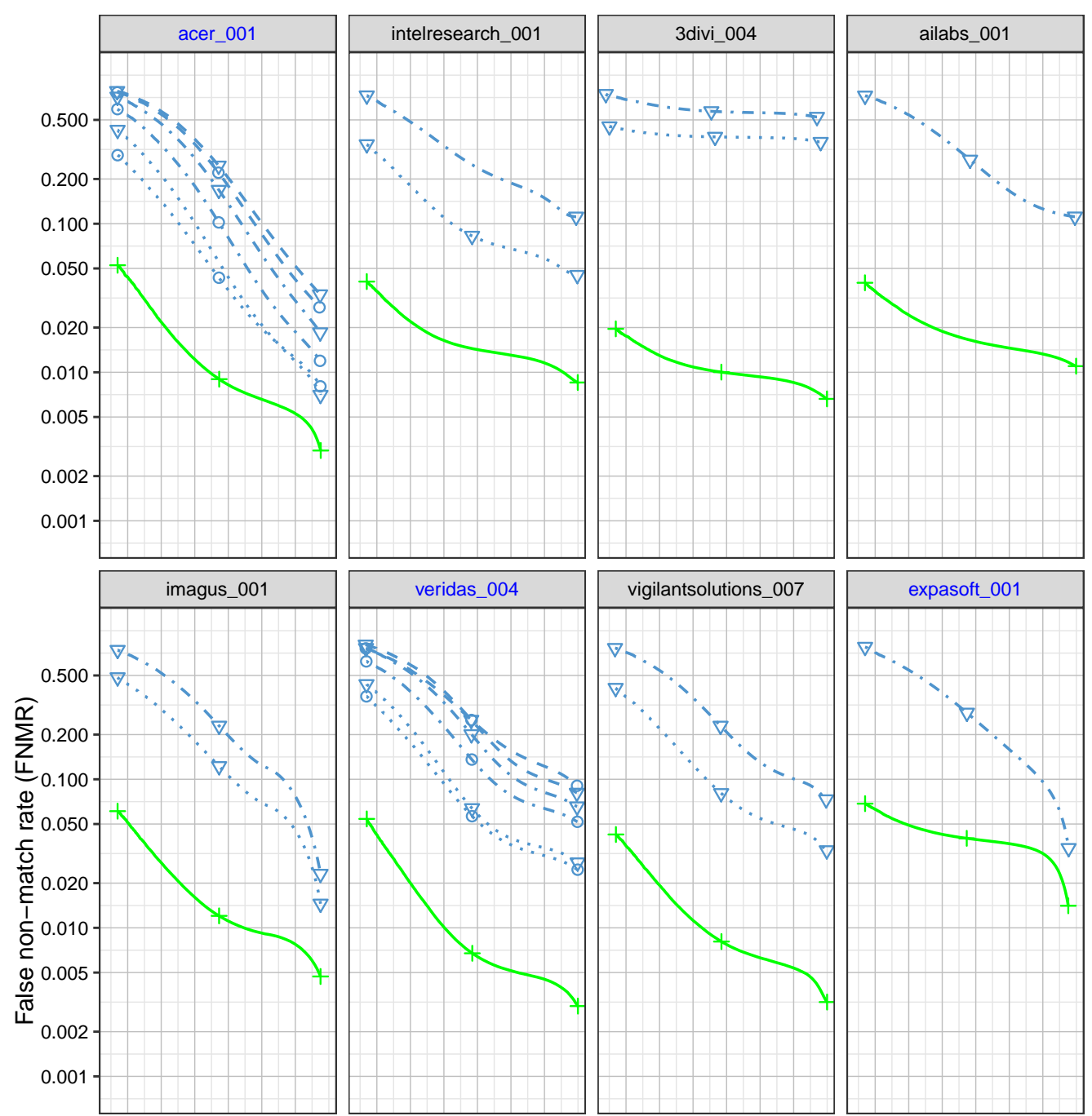

\section{Mask Nose Coverage}

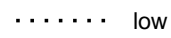

$\cdot-\cdot-\cdot$ medium

- - . high

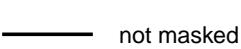

Mask Color

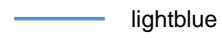

$\longrightarrow$ not masked

\section{Mask Shape}

$\nabla \quad$ wide

$\circ$ round
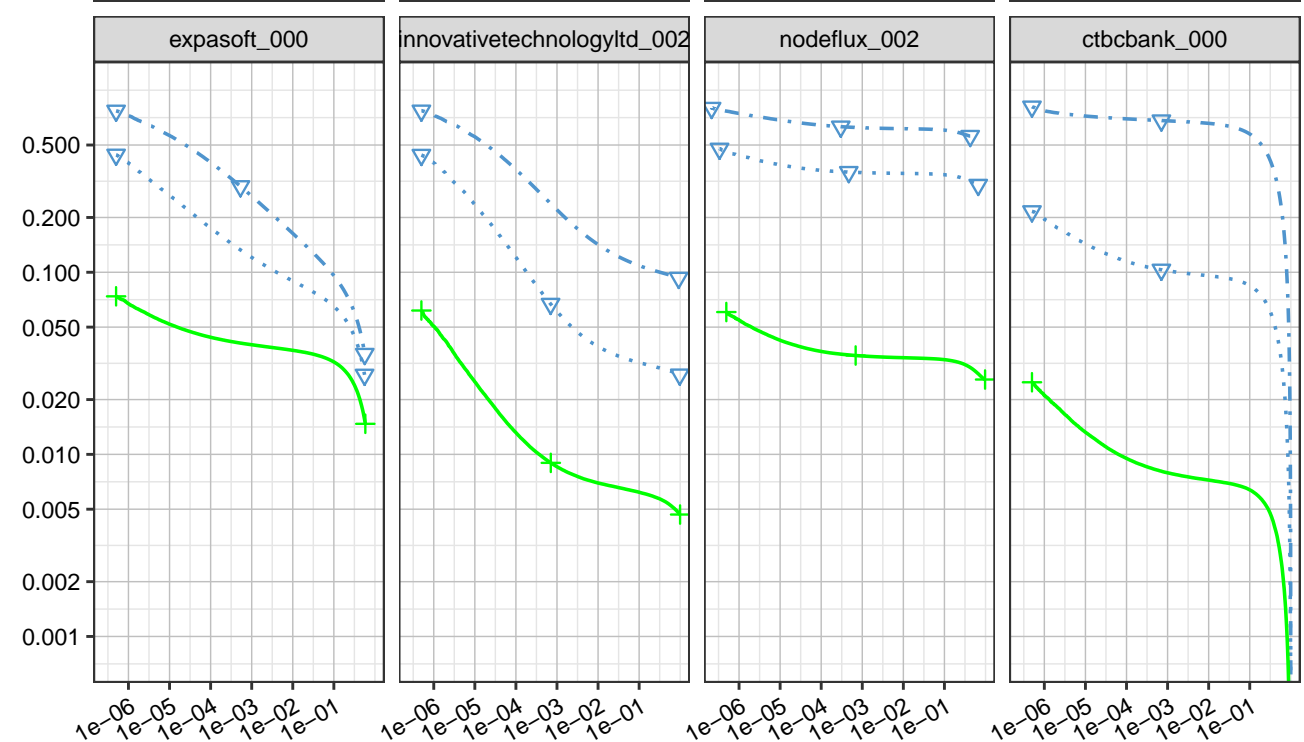

$+\quad$ not masked

False match rate (FMR)

Figure 20: DET curves showing error rates on unmasked and masked probe images, broken out by mask shape and nose coverage. Algorithms in black were submitted prior to mid-March 2020, and algorithms in blue were submitted thereafter. 

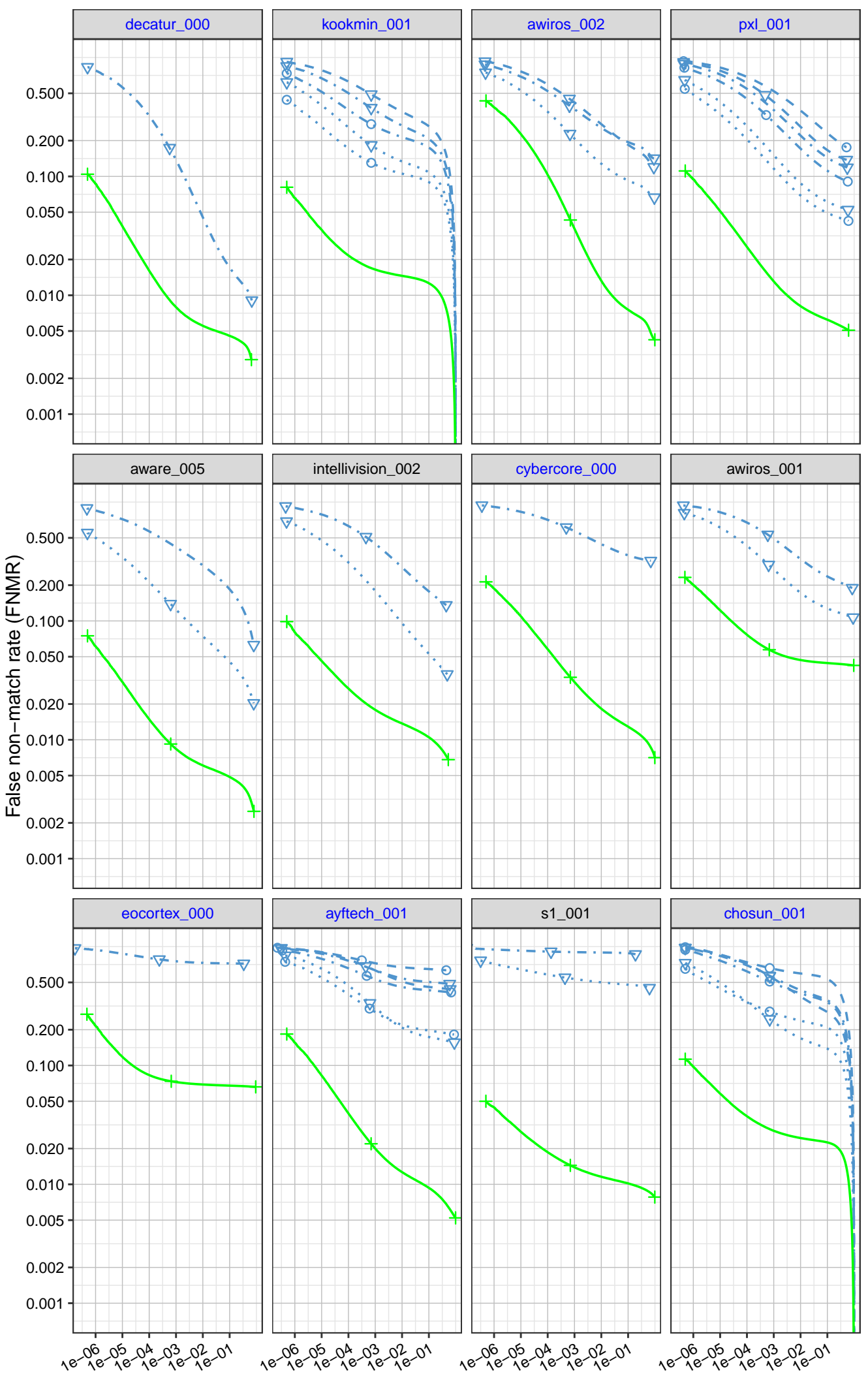

\section{Mask Nose Coverage}

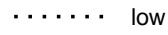

.-.-. medium

- - - high

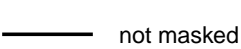

Mask Color

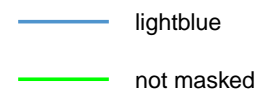

Mask Shape

$\nabla \quad$ wide

$\circ$ round
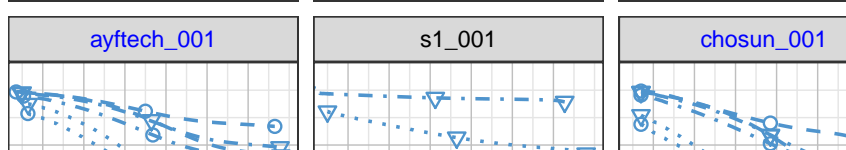

$+\quad$ not masked
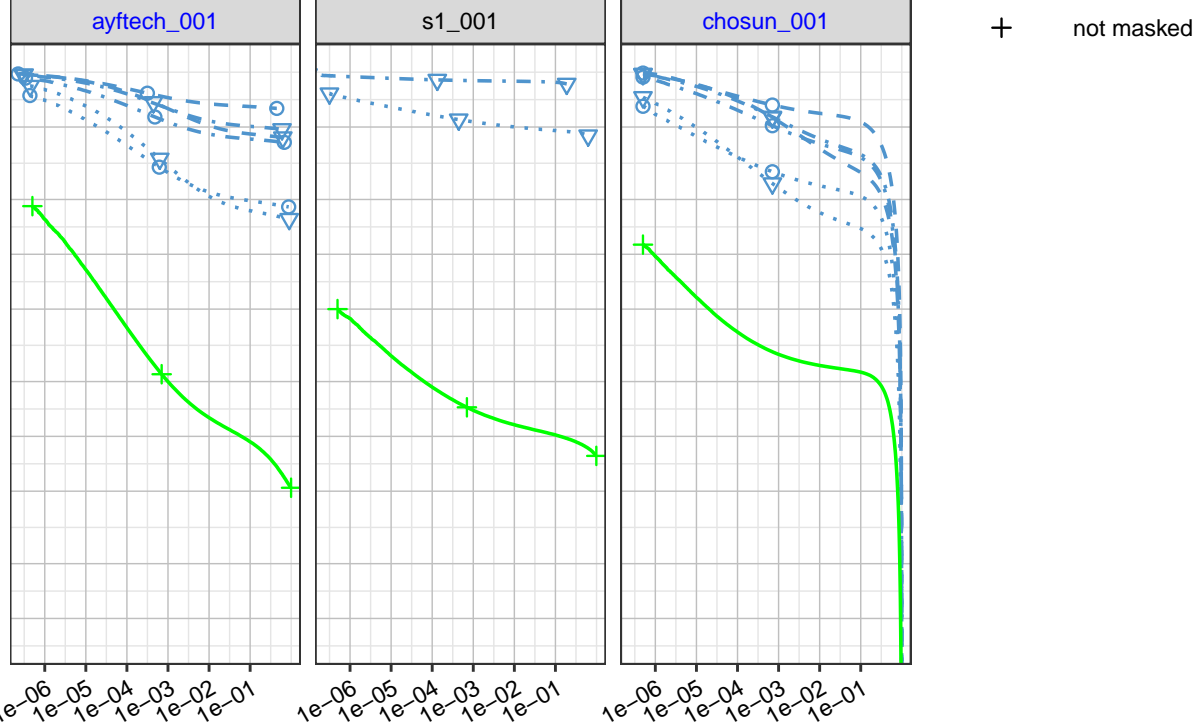

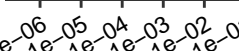
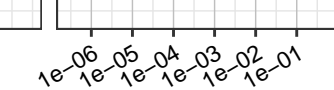

False match rate (FMR)

Figure 21: DET curves showing error rates on unmasked and masked probe images, broken out by mask shape and nose coverage. Algorithms in black were submitted prior to mid-March 2020, and algorithms in blue were submitted thereafter. 

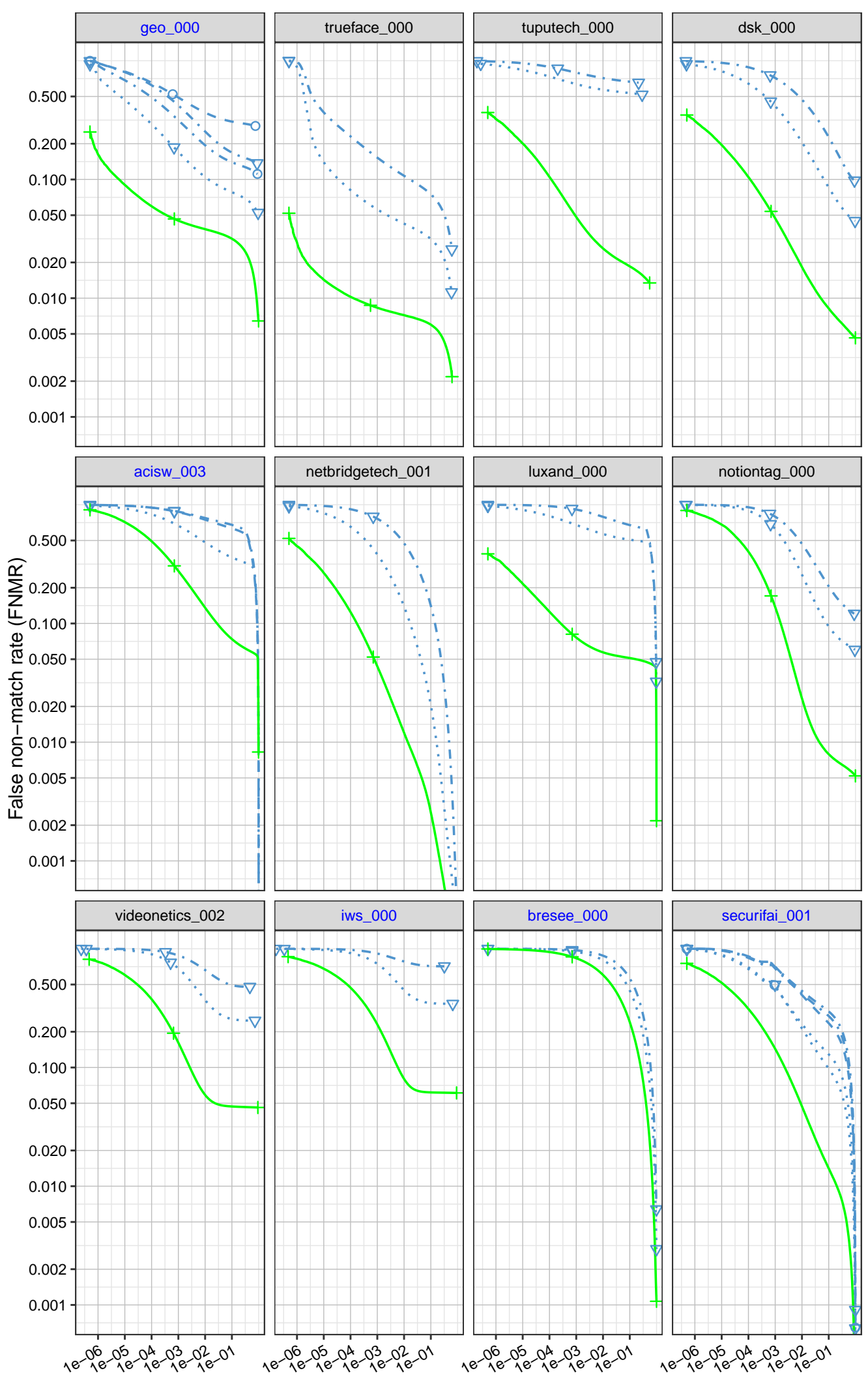

\section{Mask Nose Coverage}

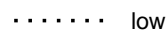

- . - . medium

- - - high

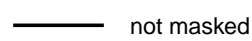

Mask Color

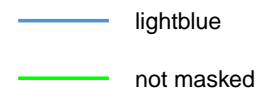

Mask Shape

$\nabla \quad$ wide

o round
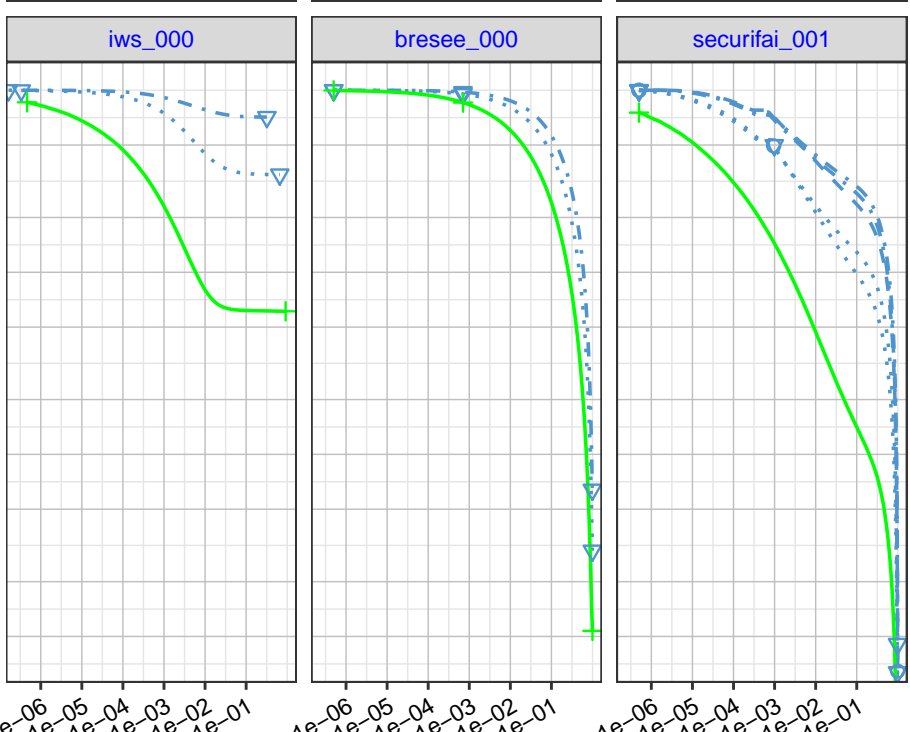

$+\quad$ not masked

False match rate (FMR)

Figure 22: DET curves showing error rates on unmasked and masked probe images, broken out by mask shape and nose coverage. Algorithms in black were submitted prior to mid-March 2020, and algorithms in blue were submitted thereafter. 

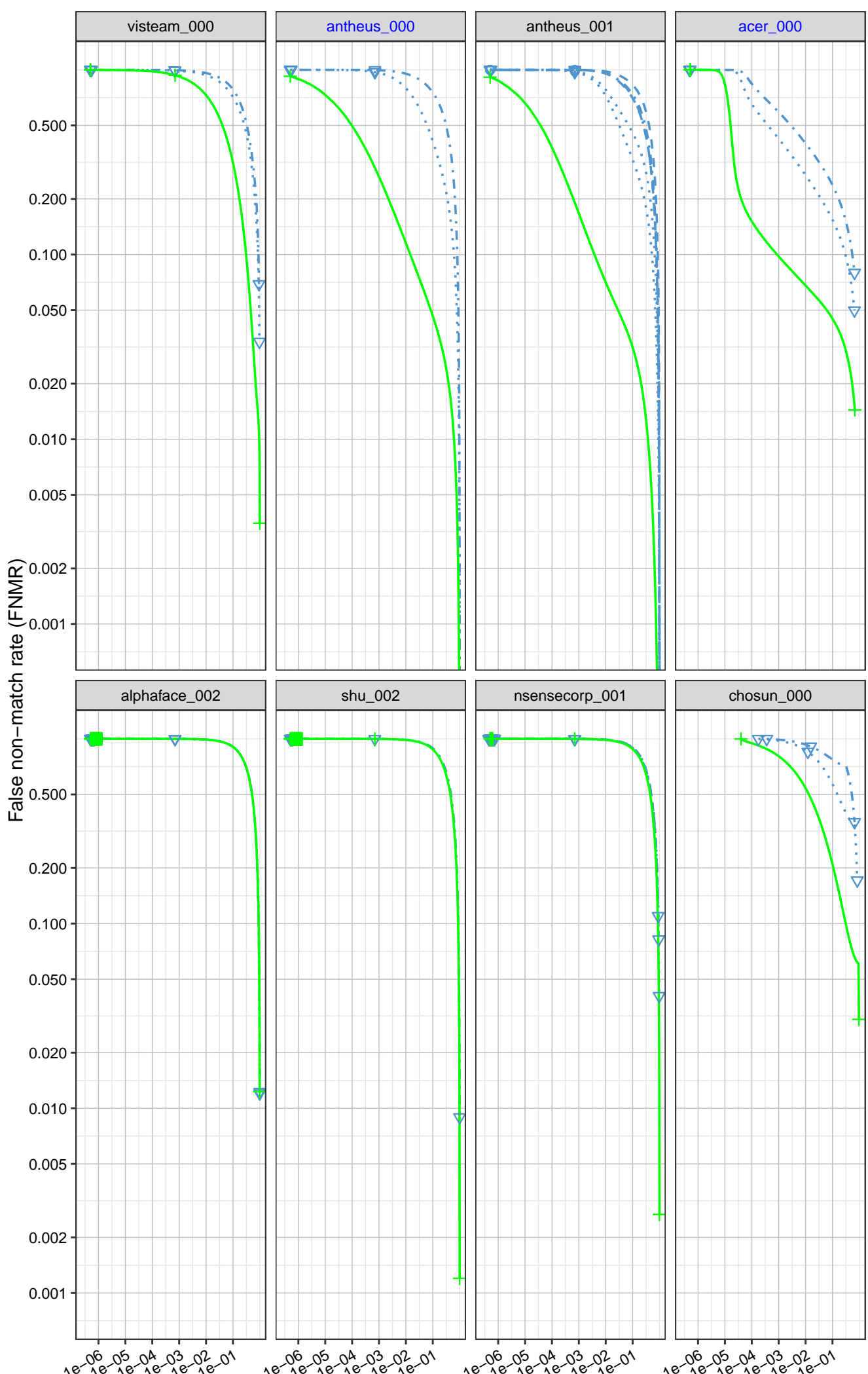

Mask Nose Coverage

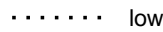

$\cdot-\cdot-\cdot$ medium

- - - high
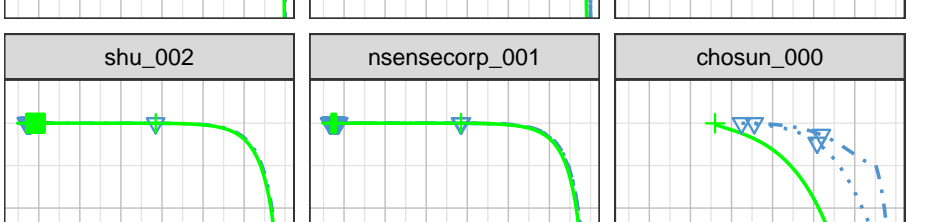

Mask Color

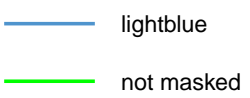

Mask Shape

$\nabla \quad$ wide

$\circ$ round

$+\quad$ not masked
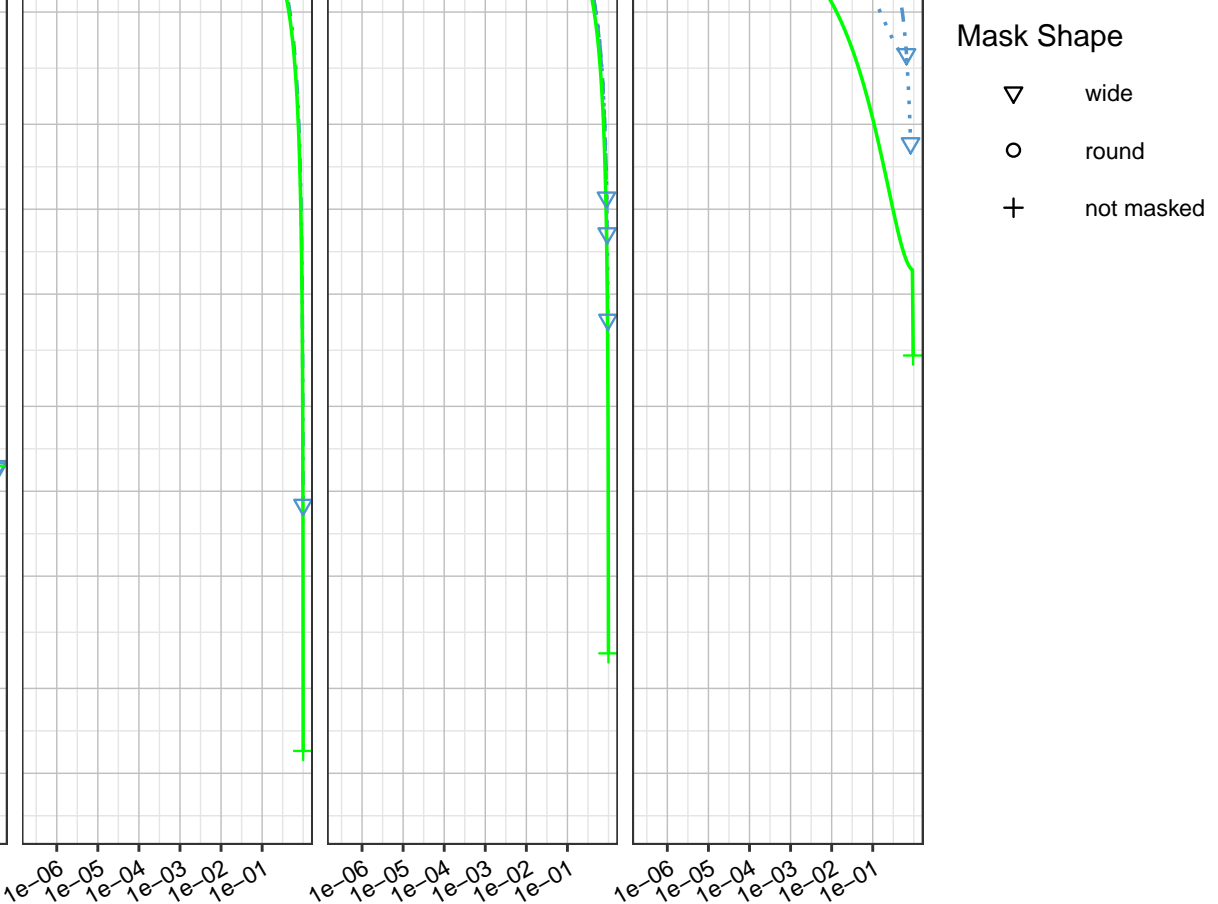

False match rate (FMR)

Figure 23: DET curves showing error rates on unmasked and masked probe images, broken out by mask shape and nose coverage. Algorithms in black were submitted prior to mid-March 2020, and algorithms in blue were submitted thereafter. 
The following plots are detection error tradeoff (DET) characteristics for each algorithm, across different mask colors. 


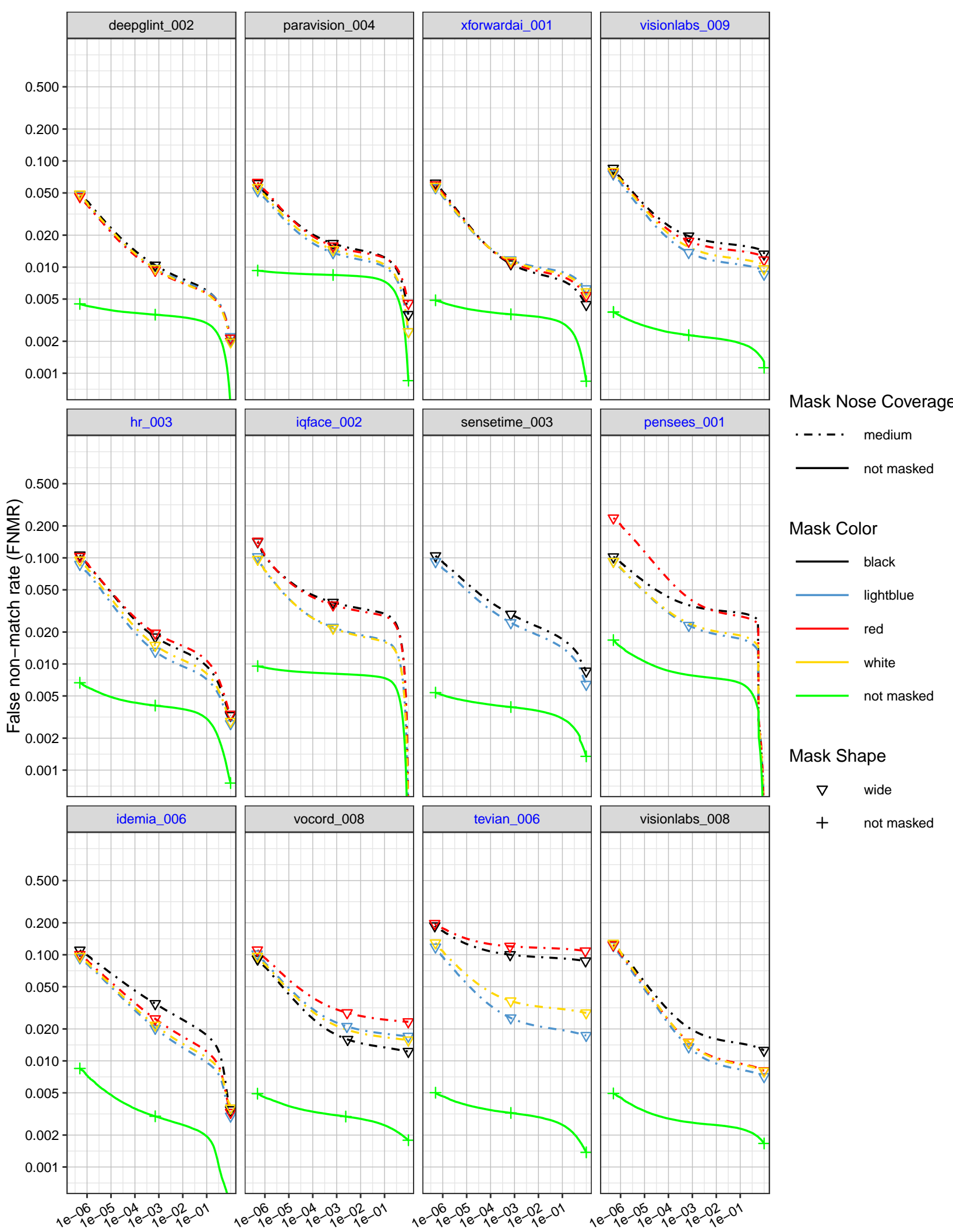

False match rate (FMR)

Figure 24: DET curves showing error rates on unmasked and masked probe images, broken out by mask color. Algorithms in black were submitted prior to mid-March 2020, and algorithms in blue were submitted thereafter. 


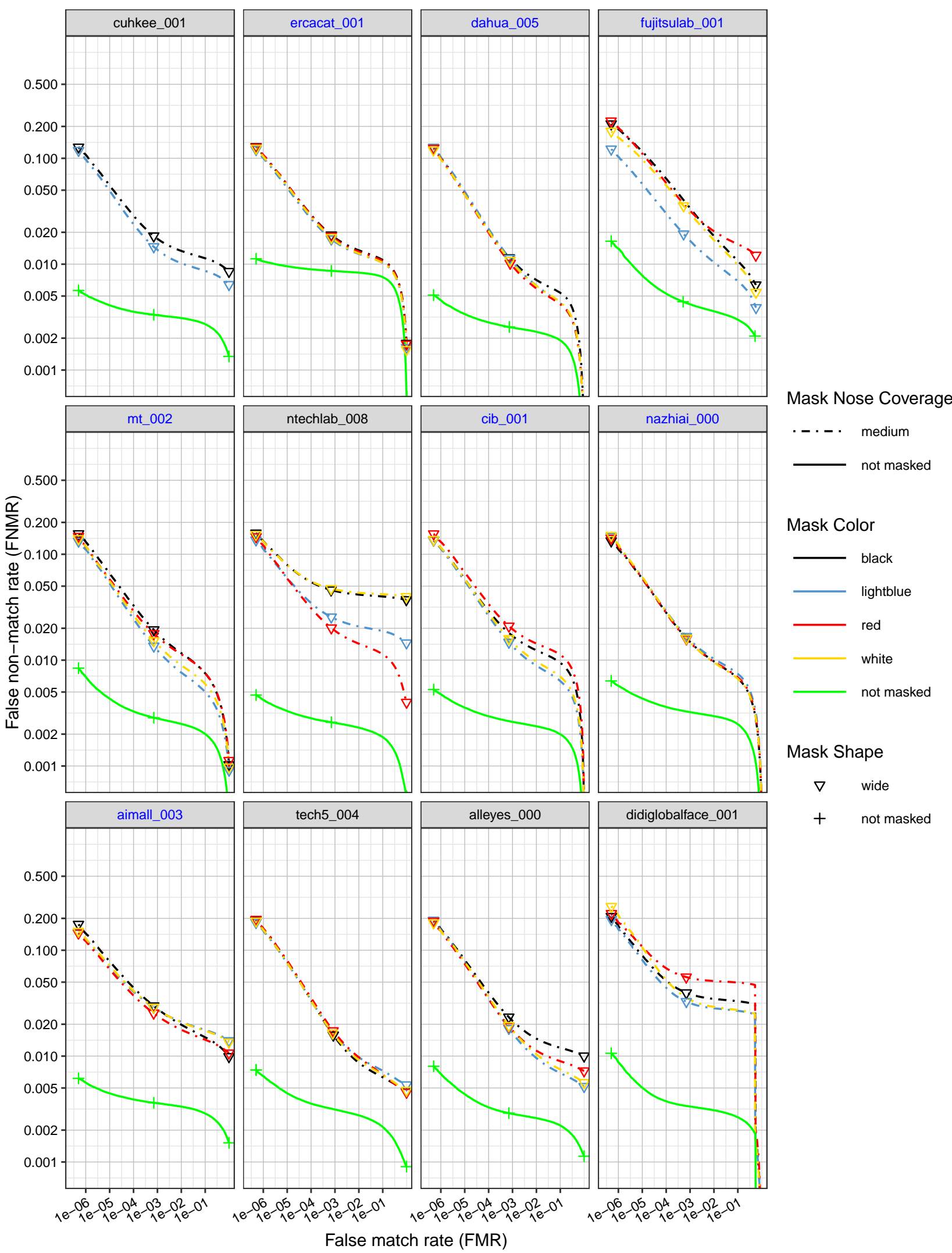

Figure 25: DET curves showing error rates on unmasked and masked probe images, broken out by mask color. Algorithms in black were submitted prior to mid-March 2020, and algorithms in blue were submitted thereafter. 


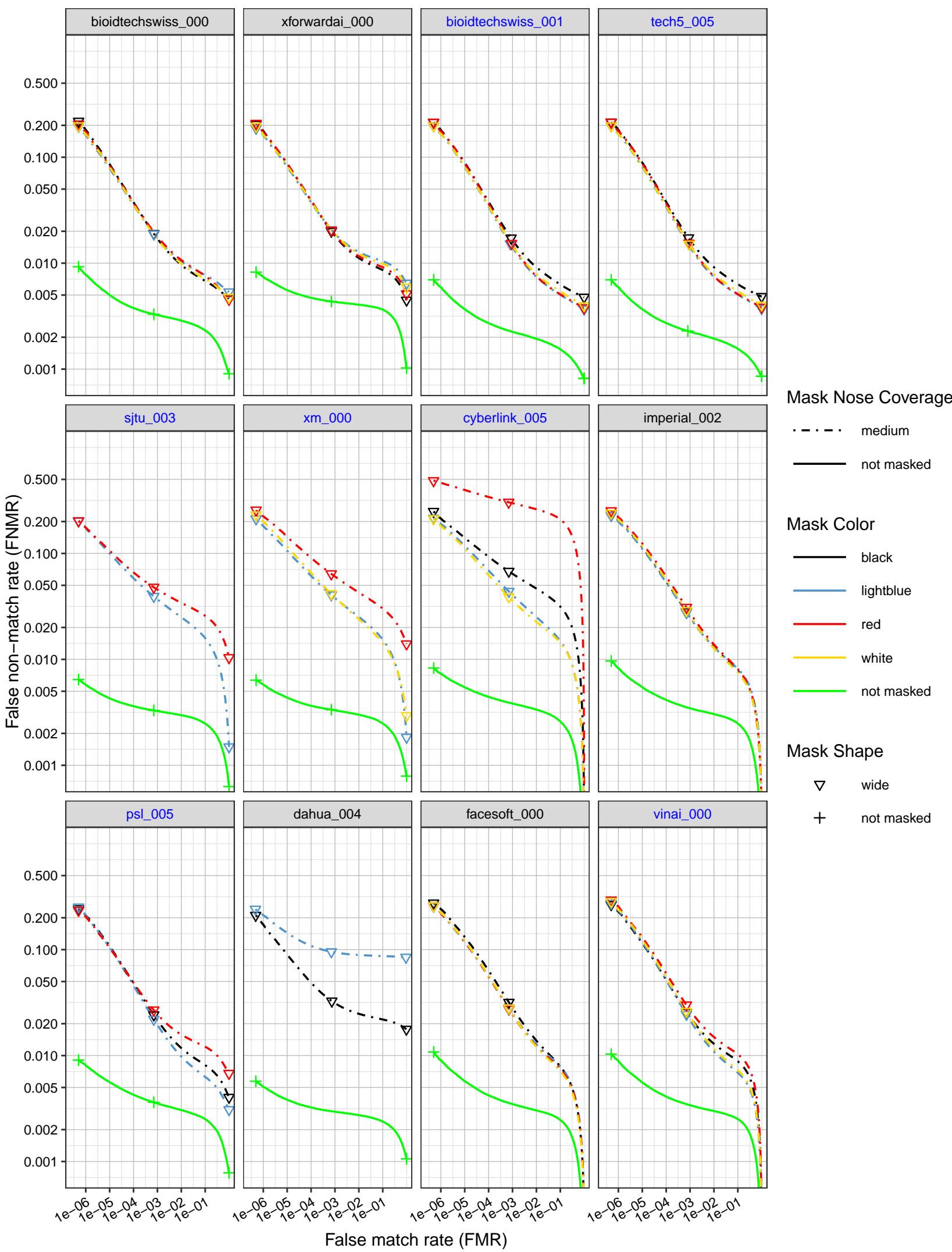

Figure 26: DET curves showing error rates on unmasked and masked probe images, broken out by mask color. Algorithms in black were submitted prior to mid-March 2020, and algorithms in blue were submitted thereafter. 


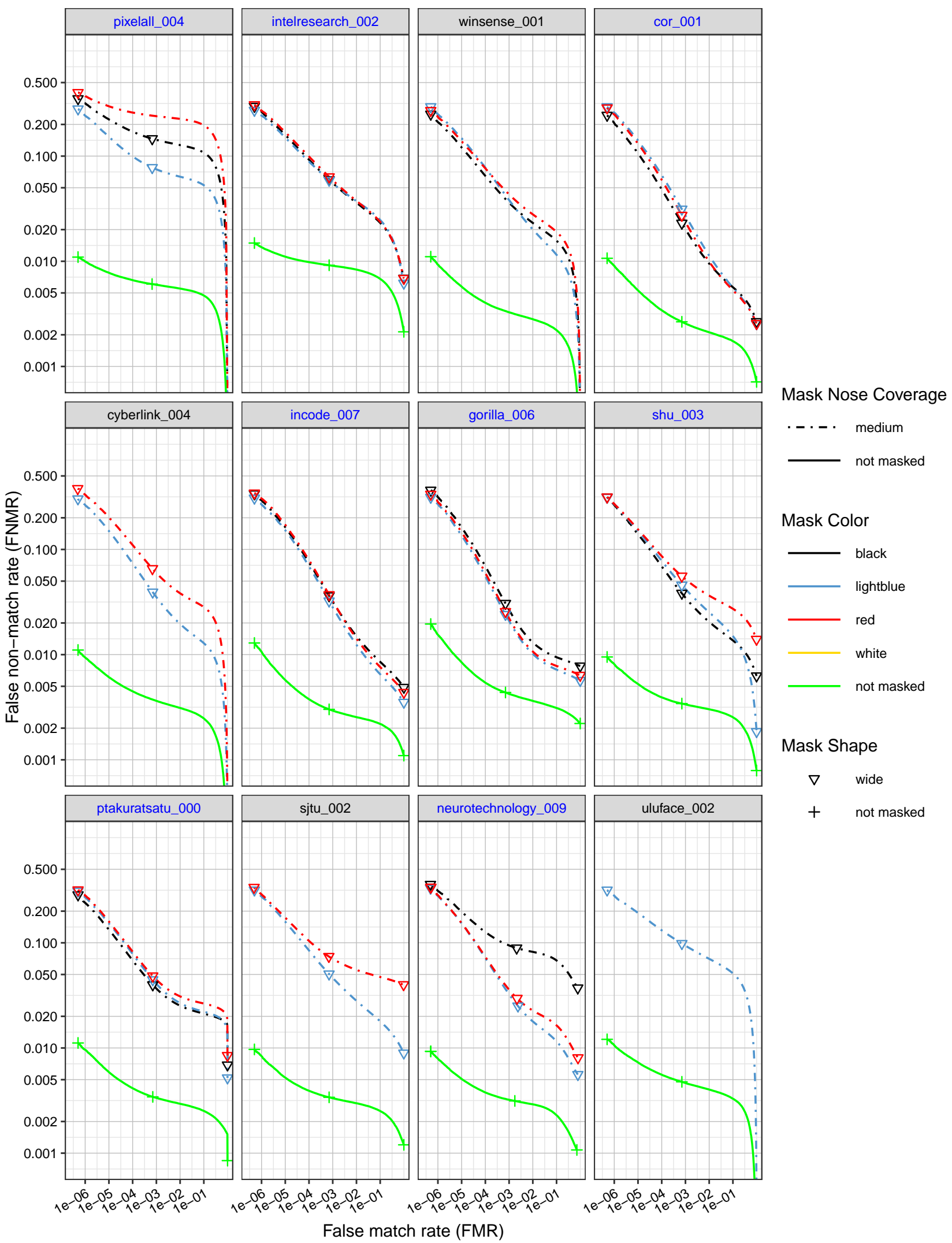

Figure 27: DET curves showing error rates on unmasked and masked probe images, broken out by mask color. Algorithms in black were submitted prior to mid-March 2020, and algorithms in blue were submitted thereafter. 

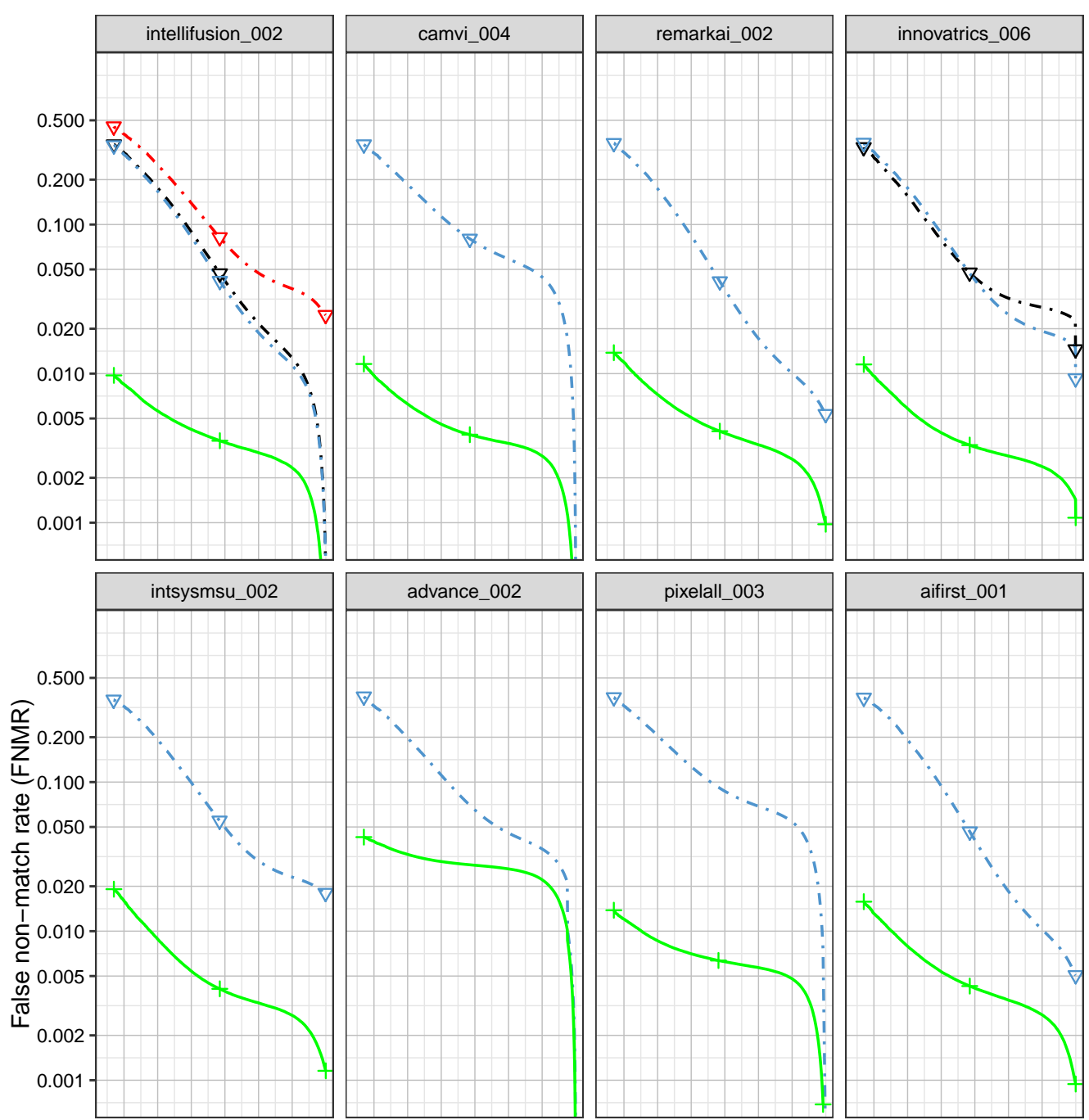

\section{Mask Nose Coverage}
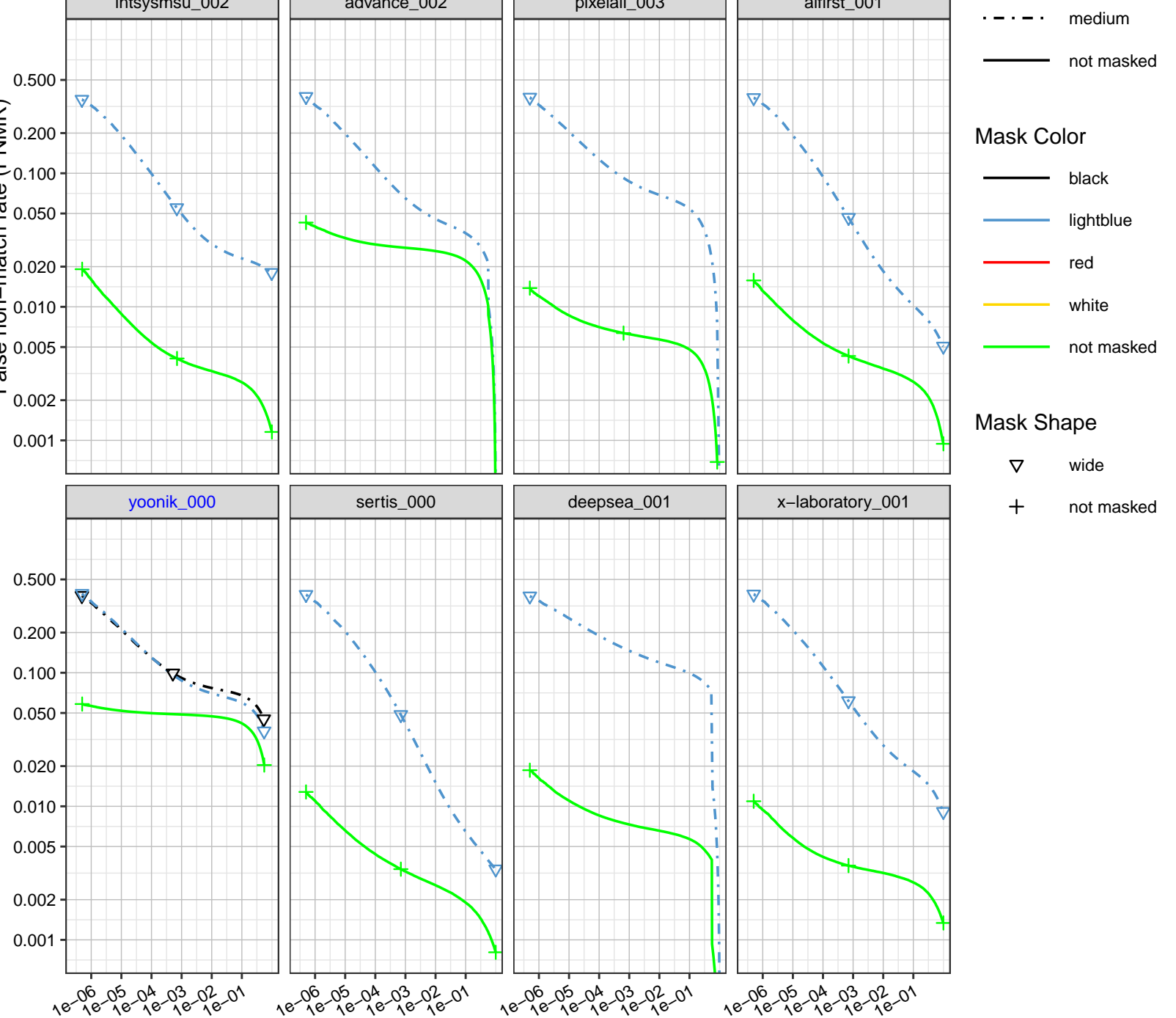

\section{Mask Color}

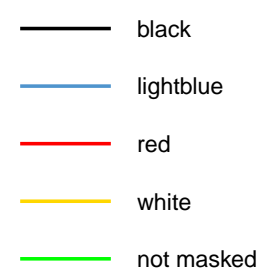

Mask Shape

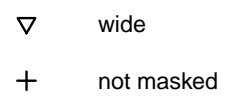

False match rate (FMR)

Figure 28: DET curves showing error rates on unmasked and masked probe images, broken out by mask color. Algorithms in black were submitted prior to mid-March 2020, and algorithms in blue were submitted thereafter. 

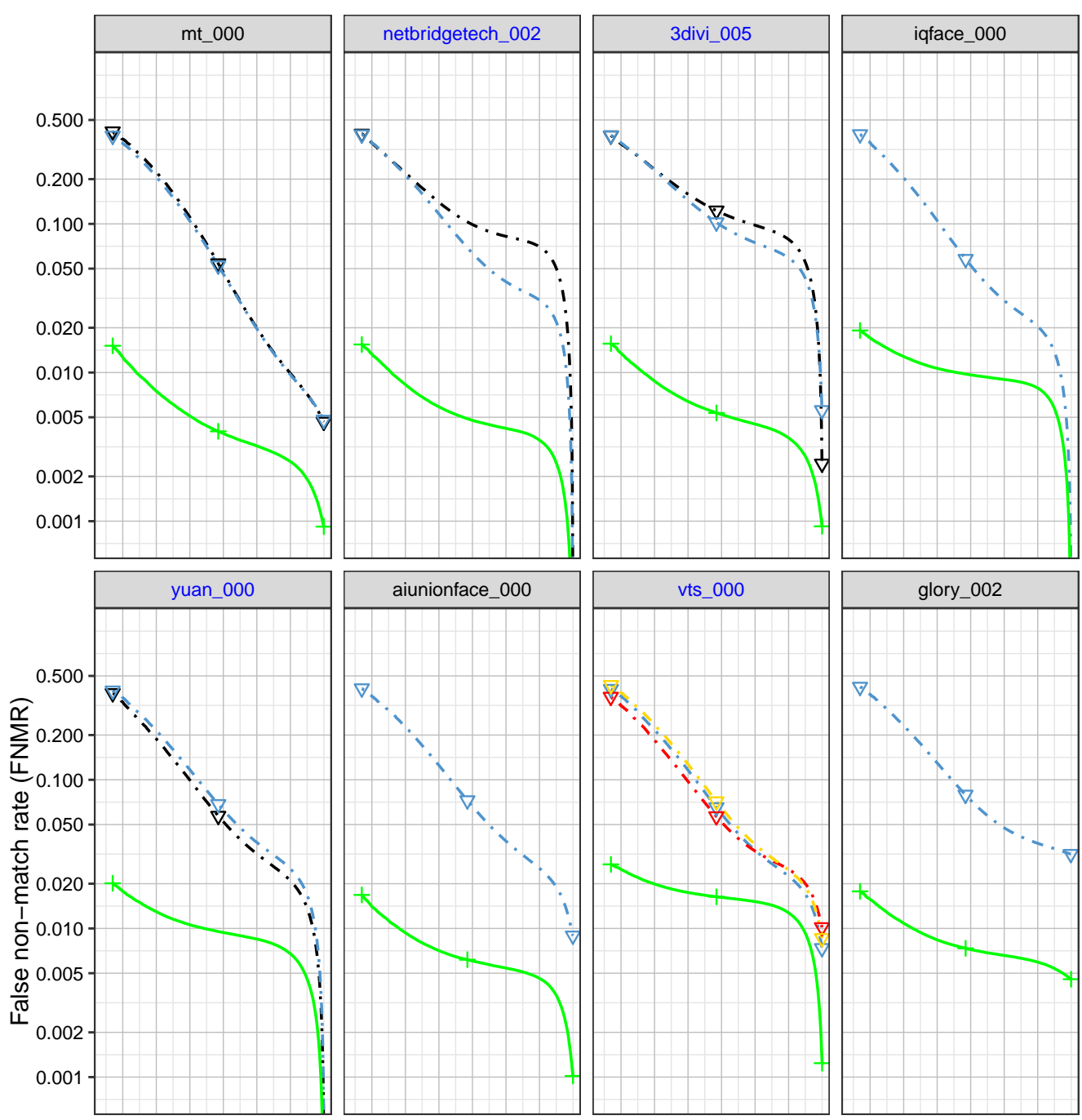

\section{Mask Nose Coverage}
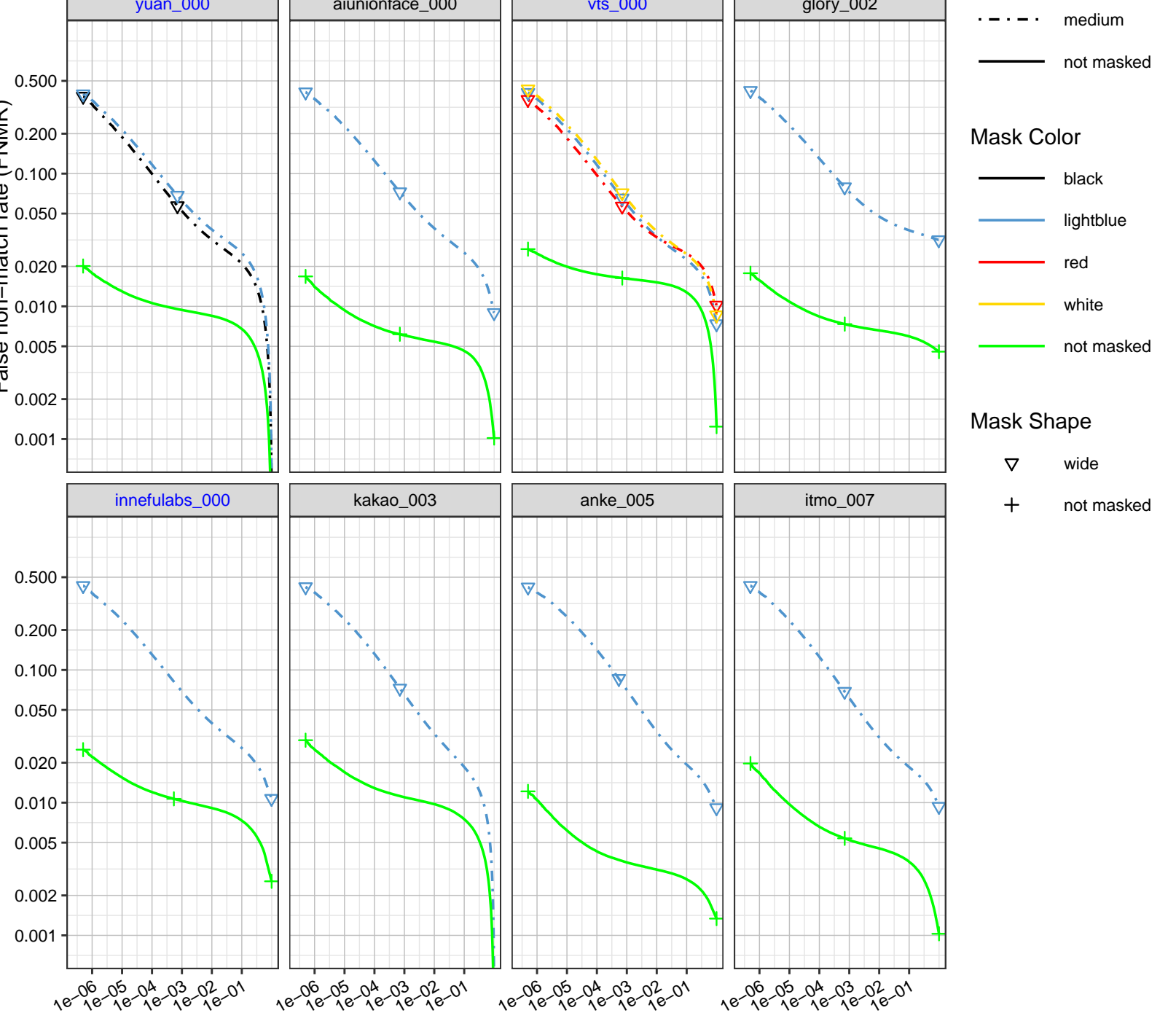

\section{Mask Color}

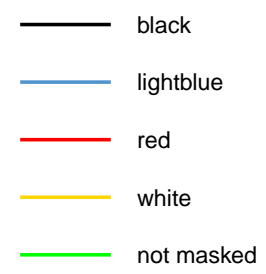

Mask Shape

$\begin{array}{ll}\nabla & \text { wide } \\ + & \text { not masked }\end{array}$

False match rate (FMR)

Figure 29: DET curves showing error rates on unmasked and masked probe images, broken out by mask color. Algorithms in black were submitted prior to mid-March 2020, and algorithms in blue were submitted thereafter. 


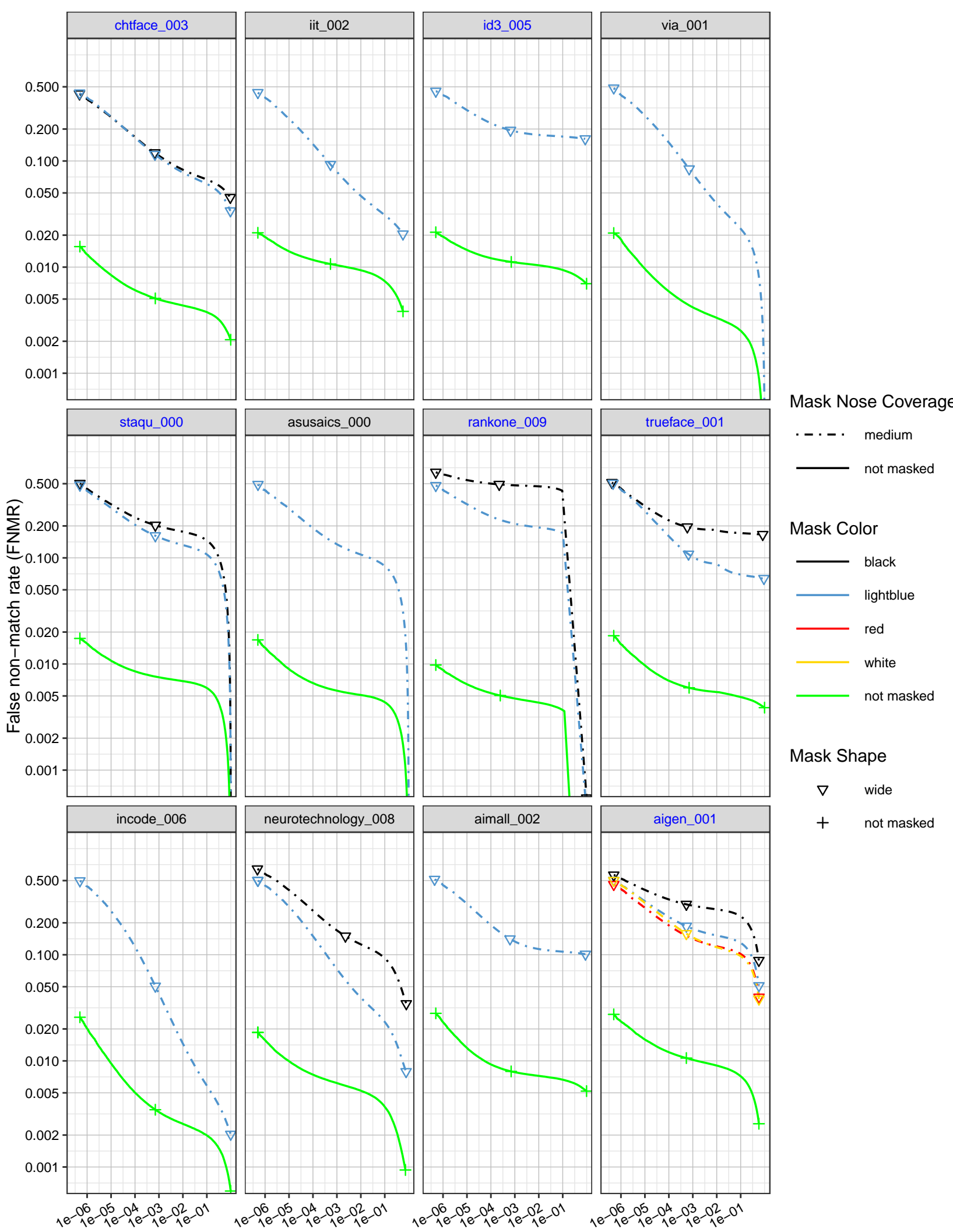

False match rate (FMR)

Figure 30: DET curves showing error rates on unmasked and masked probe images, broken out by mask color. Algorithms in black were submitted prior to mid-March 2020, and algorithms in blue were submitted thereafter. 


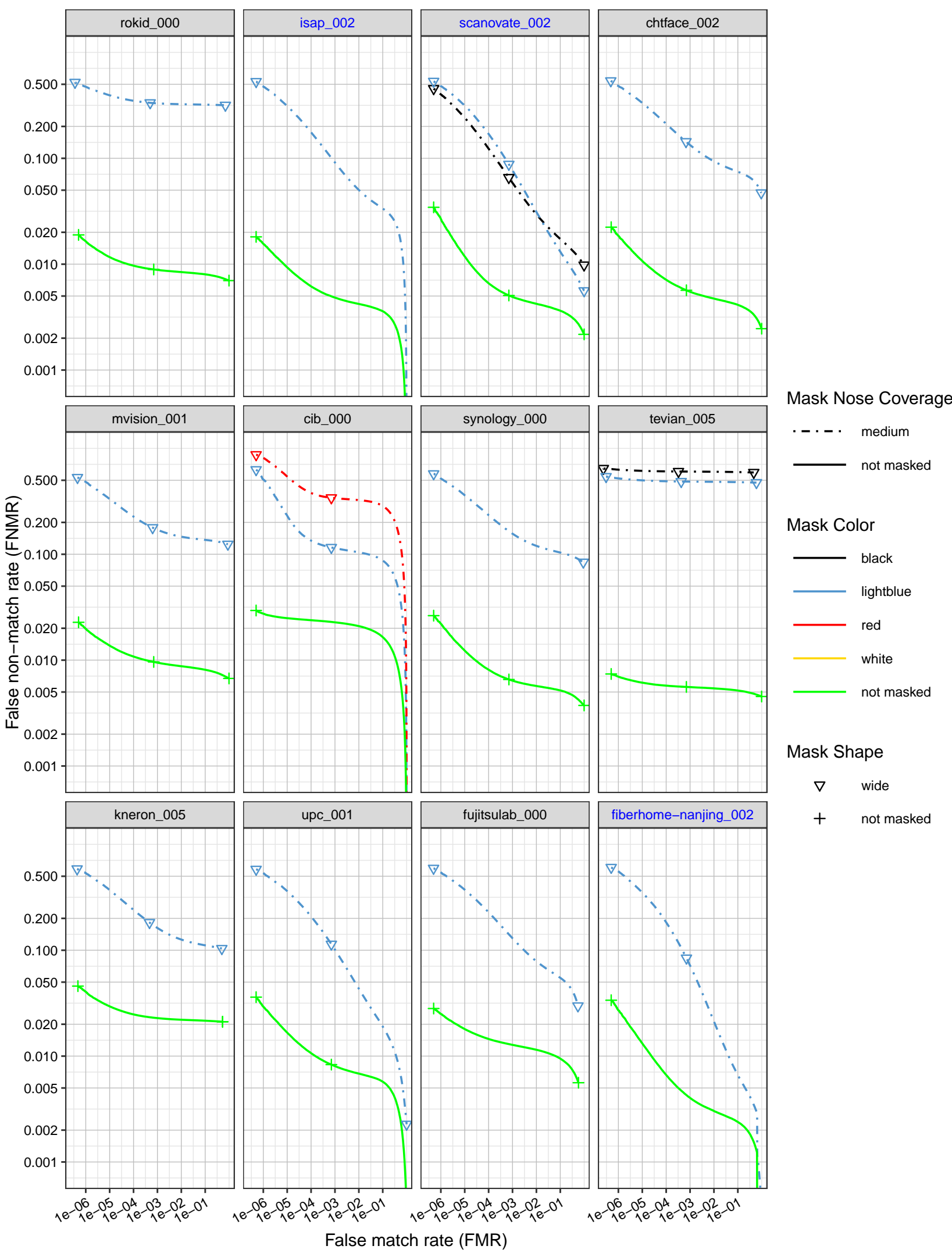

Figure 31: DET curves showing error rates on unmasked and masked probe images, broken out by mask color. Algorithms in black were submitted prior to mid-March 2020, and algorithms in blue were submitted thereafter. 


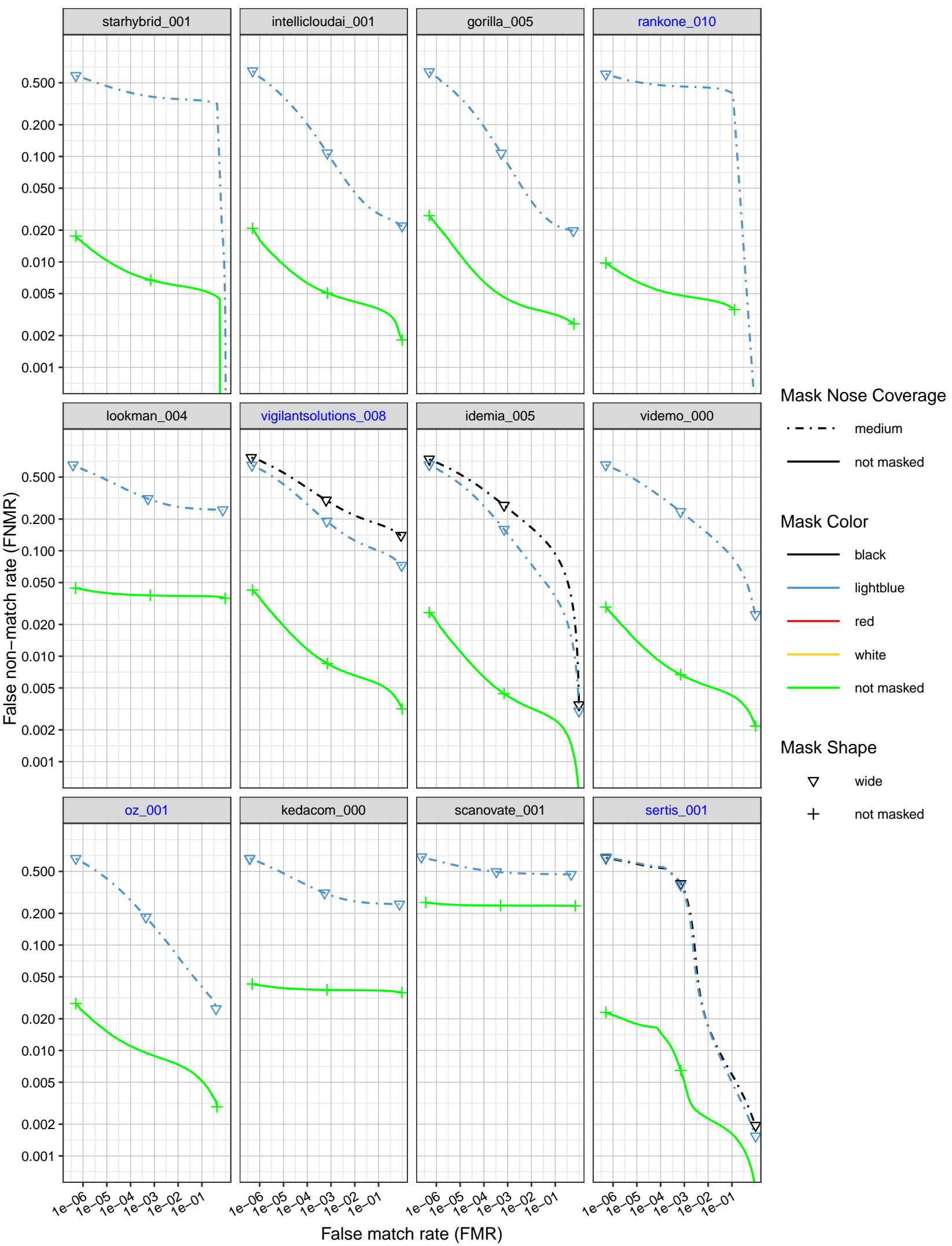

Figure 32: DET curves showing error rates on unmasked and masked probe images, broken out by mask color. Algorithms in black were submitted prior to mid-March 2020, and algorithms in blue were submitted thereafter. 


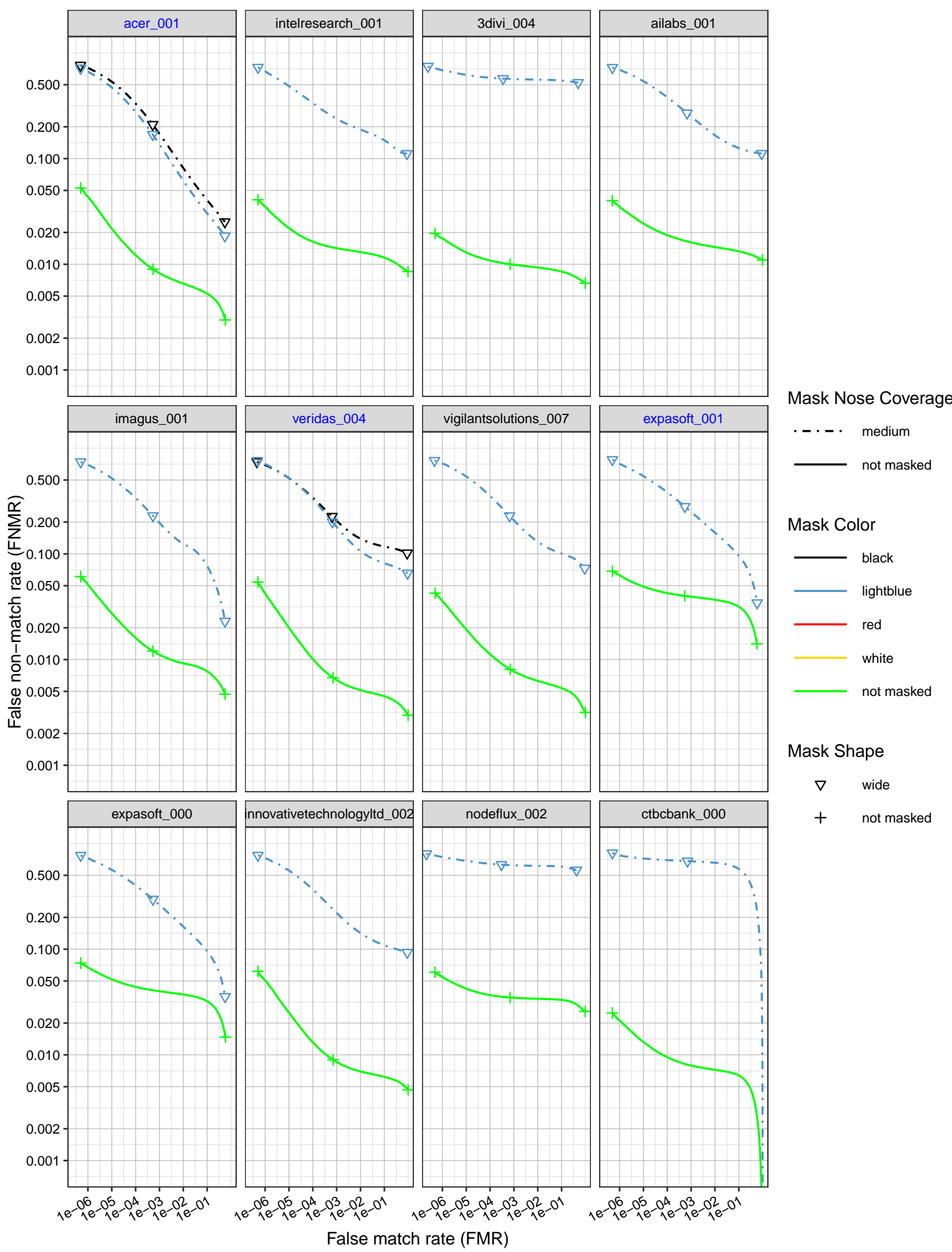

Figure 33: DET curves showing error rates on unmasked and masked probe images, broken out by mask color. Algorithms in black were submitted prior to mid-March 2020, and algorithms in blue were submitted thereafter. 

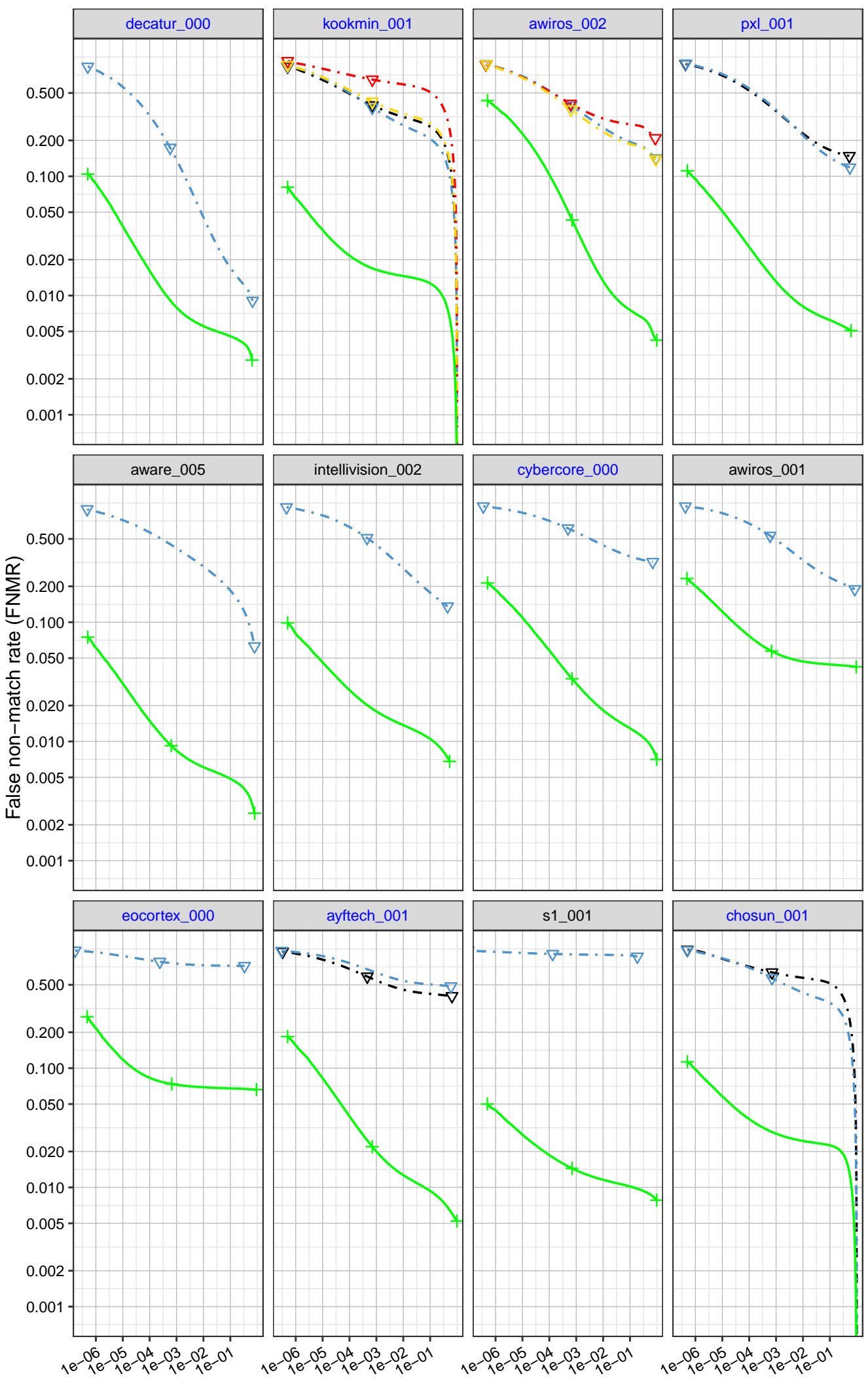

\section{Mask Nose Coverage}

.-.-. medium

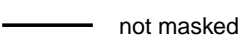

Mask Color

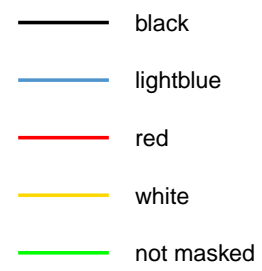

Mask Shape
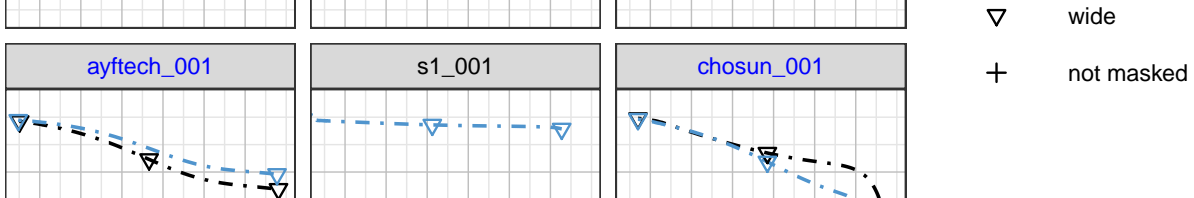

$+\quad$ not masked
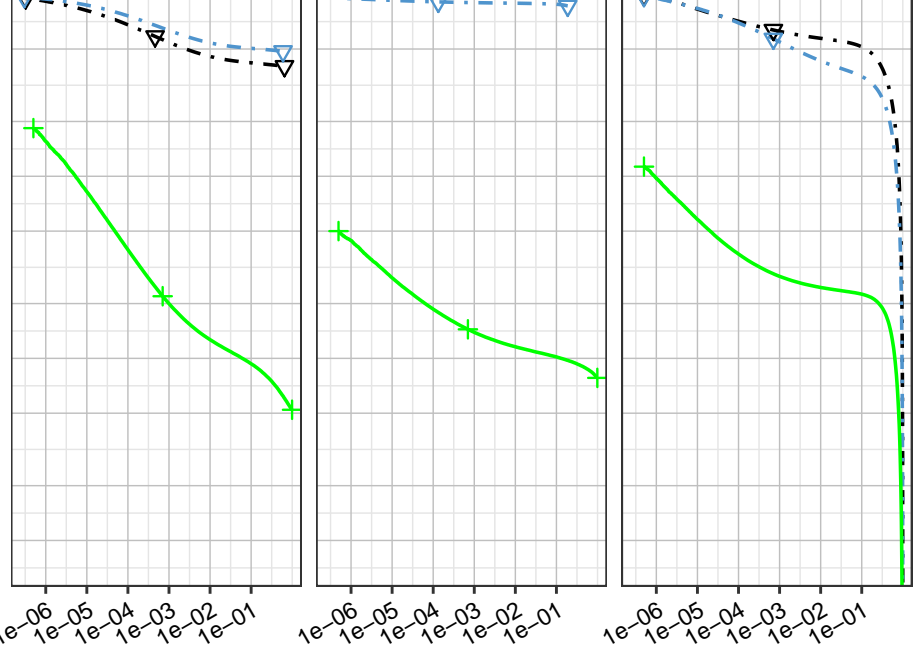

False match rate (FMR)

Figure 34: DET curves showing error rates on unmasked and masked probe images, broken out by mask color. Algorithms in black were submitted prior to mid-March 2020, and algorithms in blue were submitted thereafter. 


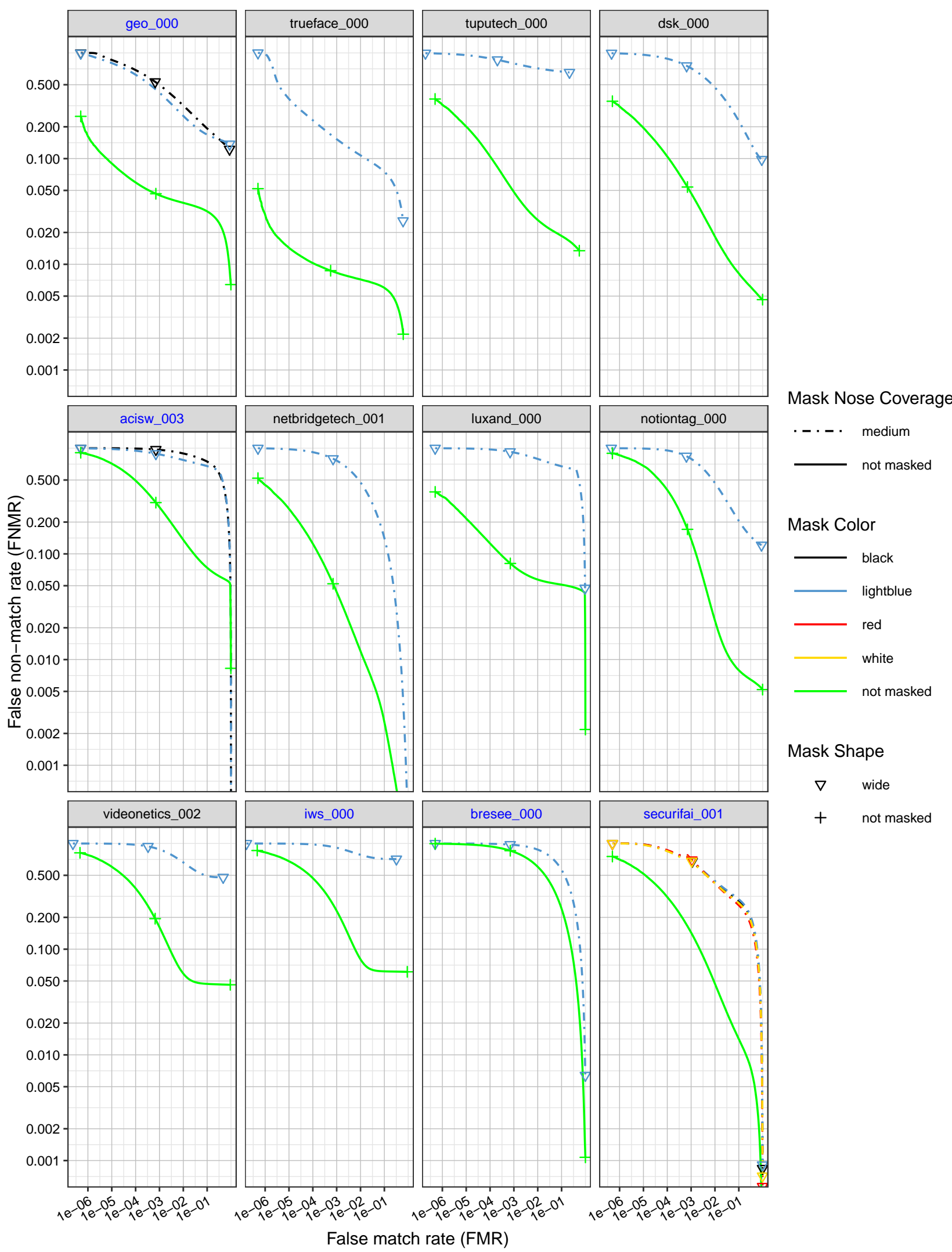

Figure 35: DET curves showing error rates on unmasked and masked probe images, broken out by mask color. Algorithms in black were submitted prior to mid-March 2020, and algorithms in blue were submitted thereafter. 

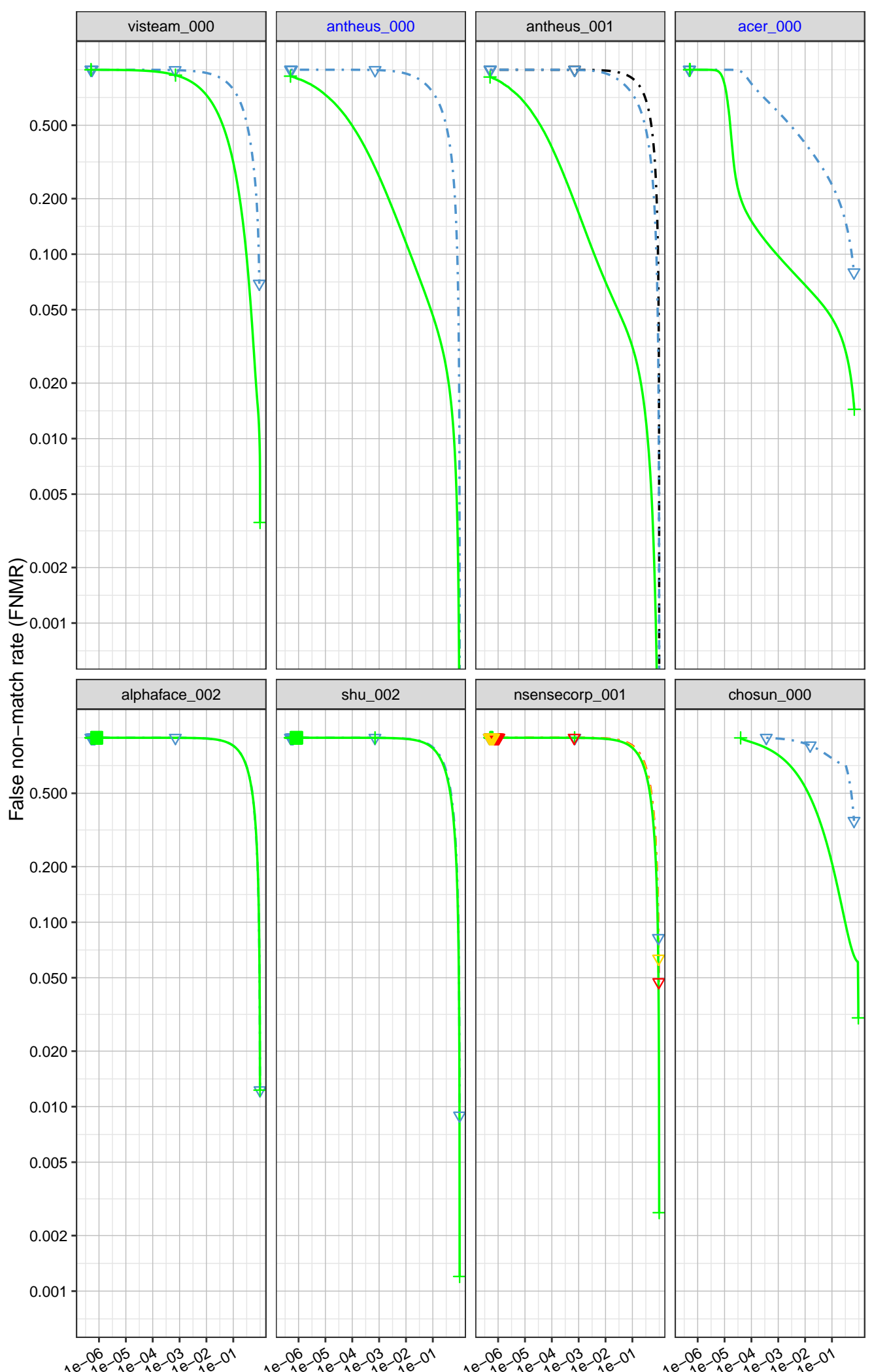

Mask Nose Coverage

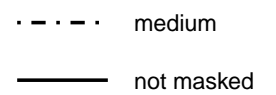

\section{Mask Color}
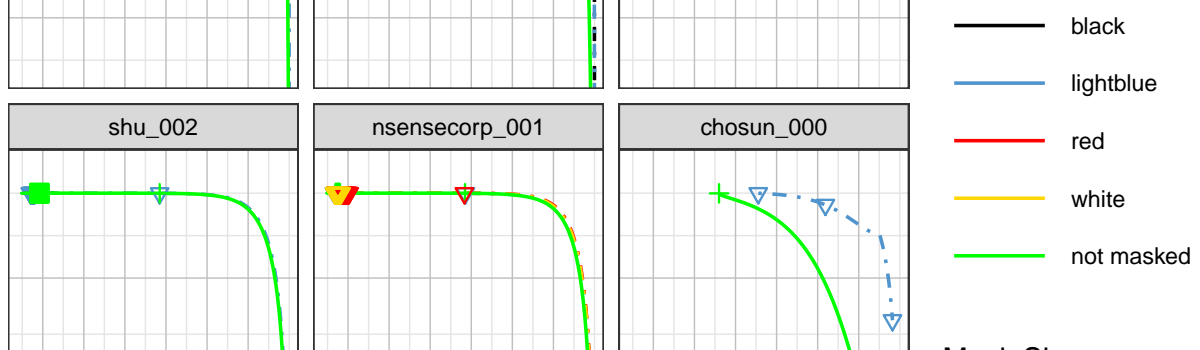

Mask Shape

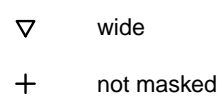

$+\quad$ not masked

Figure 36: DET curves showing error rates on unmasked and masked probe images, broken out by mask color. Algorithms in black were submitted prior to mid-March 2020, and algorithms in blue were submitted thereafter. 
The following plots show the explicit dependence of false non-match rate (FNMR) and false match rate (FMR) on score threshold for each algorithm, across different masked/unmasked combinations. 

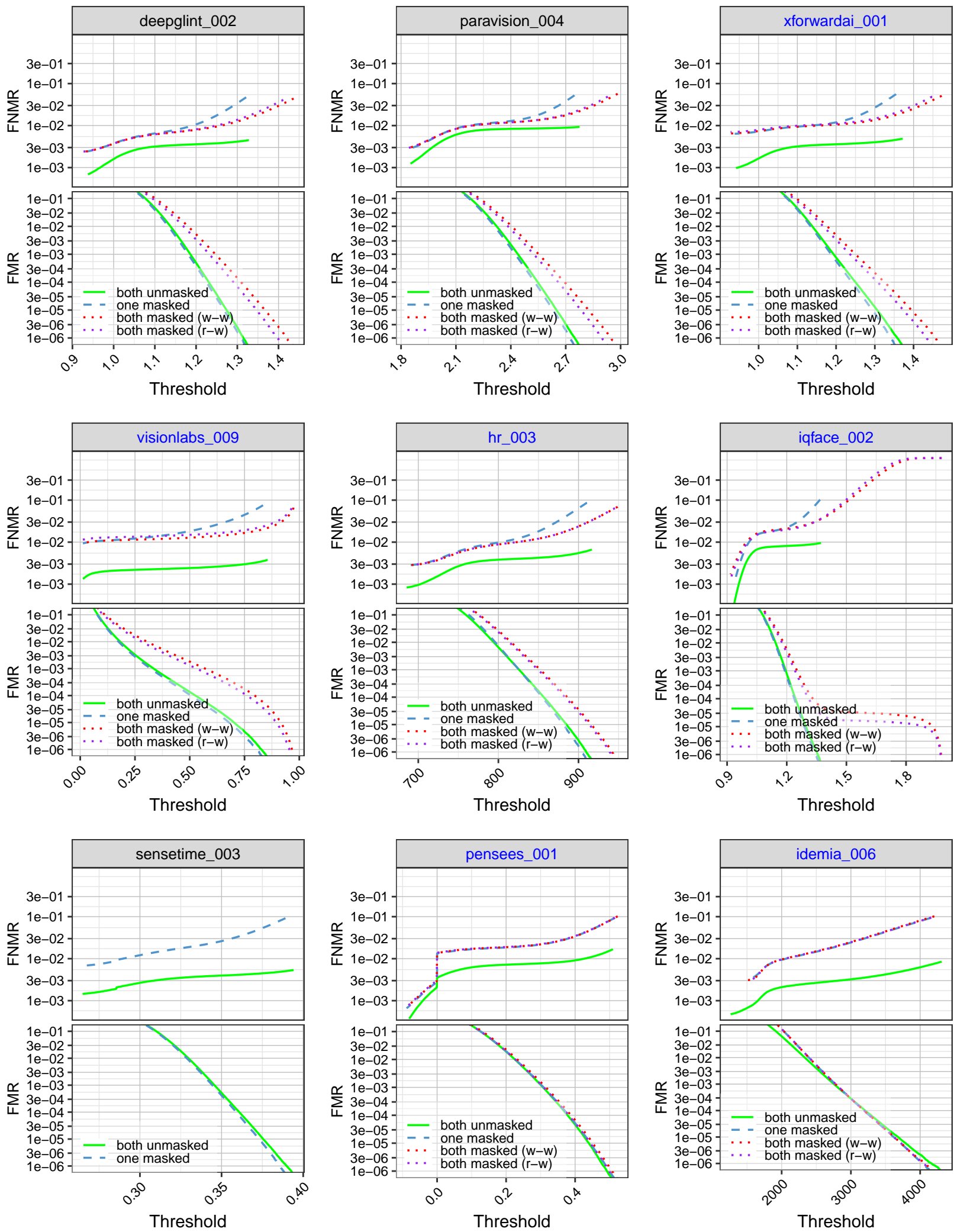

Figure 37: FNMR and FMR calibration curves on unmasked-to-unmasked (both unmasked), masked-to-unmasked with medium, wide, lightblue masks (one masked), masked-to-masked with medium, wide, lightblue masks (both masked (w-w)), and masked-tomasked with medium, round, white masks (enrollment image) and medium, wide, lightblue masks (verification image) (both masked $(r-w))$. Algorithms in black were submitted prior to mid-March 2020, and algorithms in blue were submitted thereafter. 

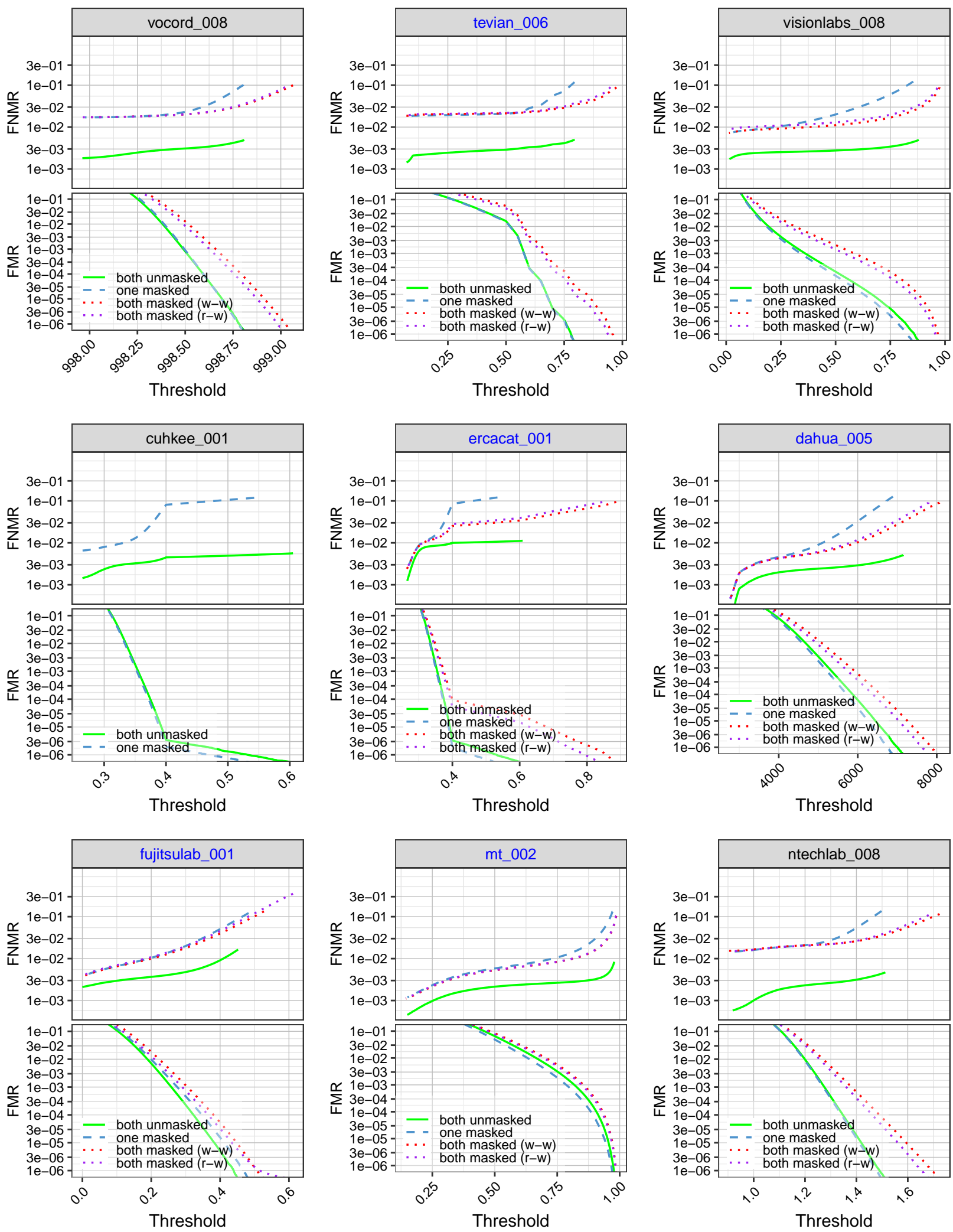

Figure 38: FNMR and FMR calibration curves on unmasked-to-unmasked (both unmasked), masked-to-unmasked with medium, wide, lightblue masks (one masked), masked-to-masked with medium, wide, lightblue masks (both masked (w-w)), and masked-tomasked with medium, round, white masks (enrollment image) and medium, wide, lightblue masks (verification image) (both masked $(r-w))$. Algorithms in black were submitted prior to mid-March 2020, and algorithms in blue were submitted thereafter. 

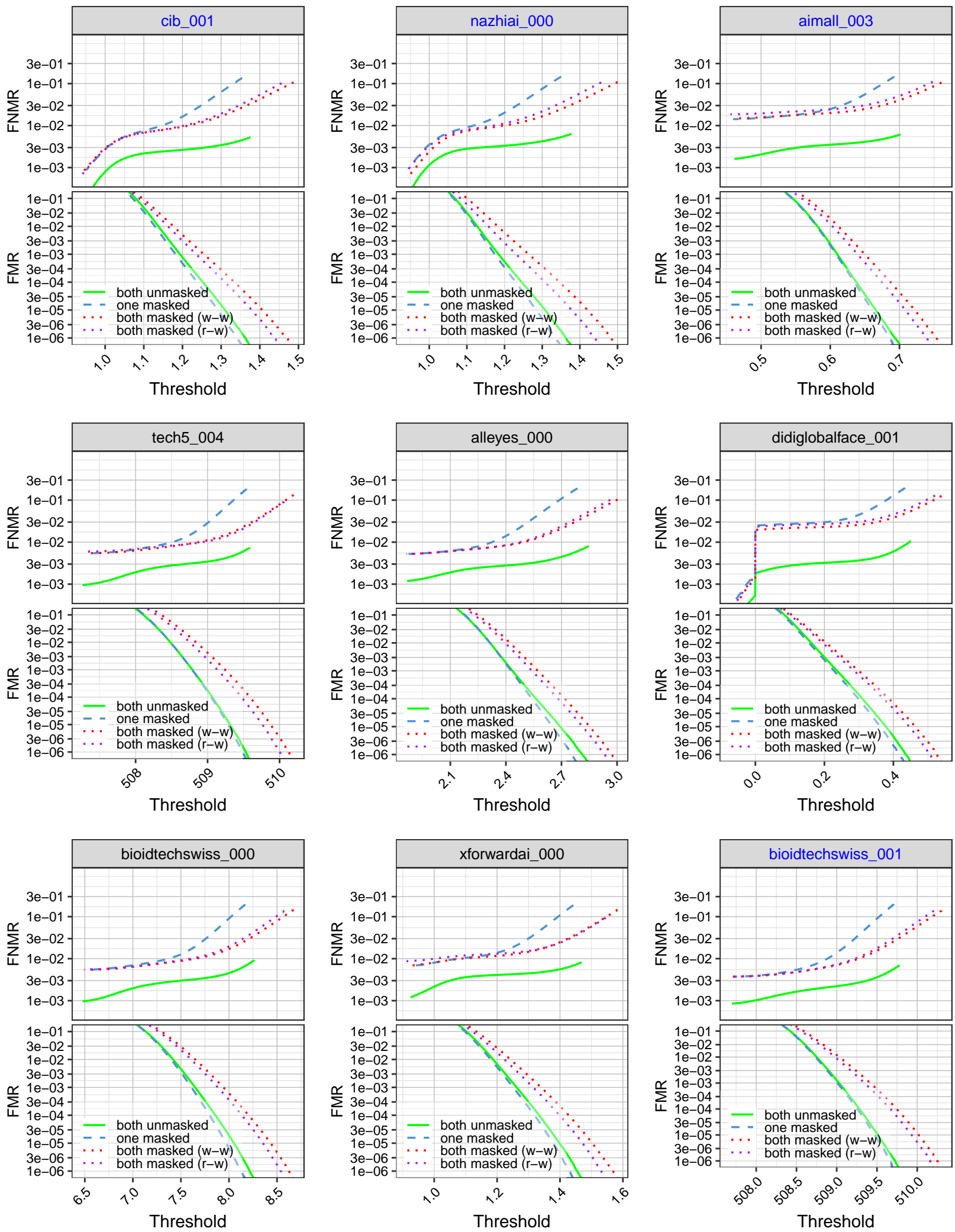

Figure 39: FNMR and FMR calibration curves on unmasked-to-unmasked (both unmasked), masked-to-unmasked with medium, wide, lightblue masks (one masked), masked-to-masked with medium, wide, lightblue masks (both masked (w-w)), and masked-tomasked with medium, round, white masks (enrollment image) and medium, wide, lightblue masks (verification image) (both masked $(r-w))$. Algorithms in black were submitted prior to mid-March 2020, and algorithms in blue were submitted thereafter. 

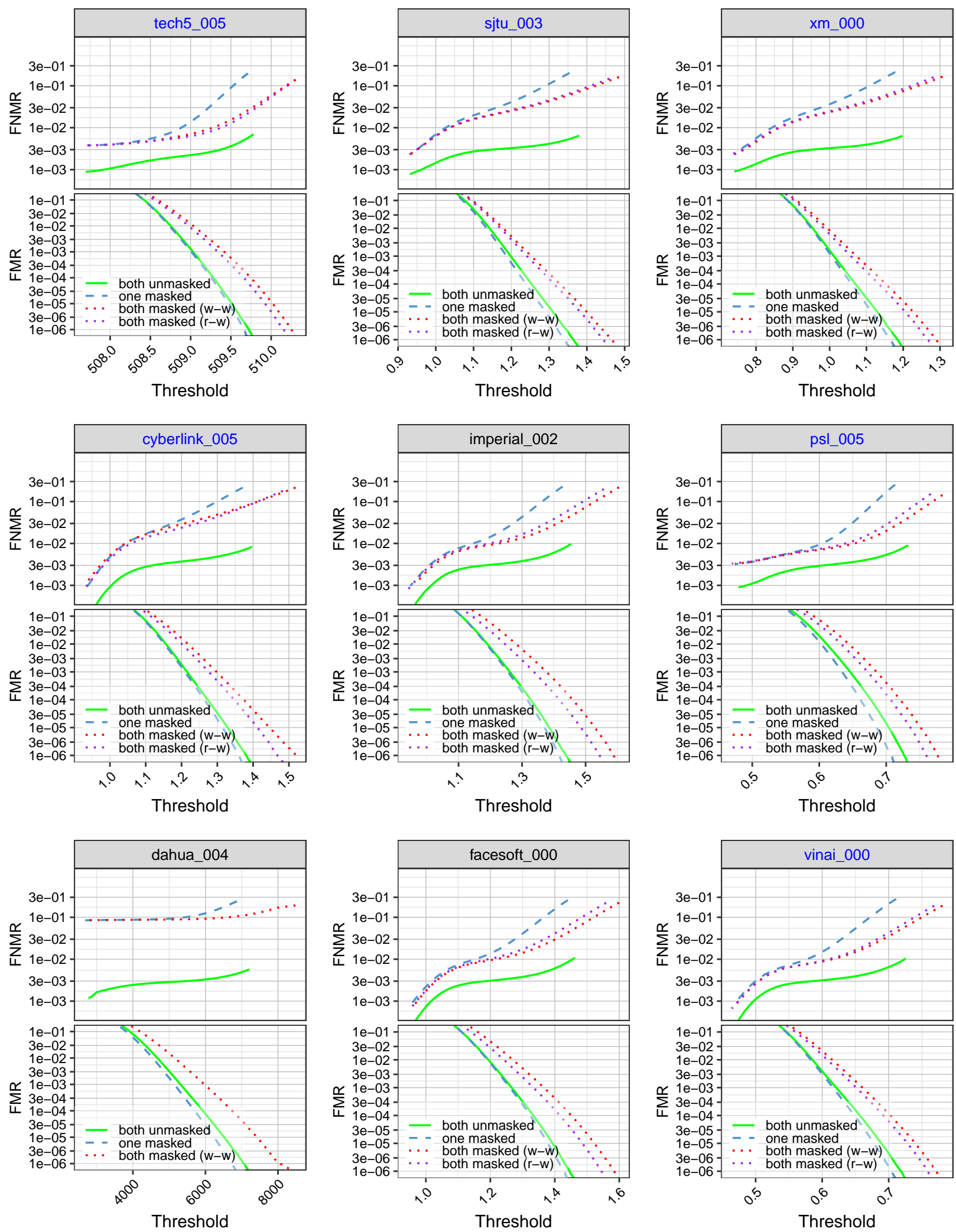

Figure 40: FNMR and FMR calibration curves on unmasked-to-unmasked (both unmasked), masked-to-unmasked with medium, wide, lightblue masks (one masked), masked-to-masked with medium, wide, lightblue masks (both masked (w-w)), and masked-tomasked with medium, round, white masks (enrollment image) and medium, wide, lightblue masks (verification image) (both masked $(r-w))$. Algorithms in black were submitted prior to mid-March 2020, and algorithms in blue were submitted thereafter. 

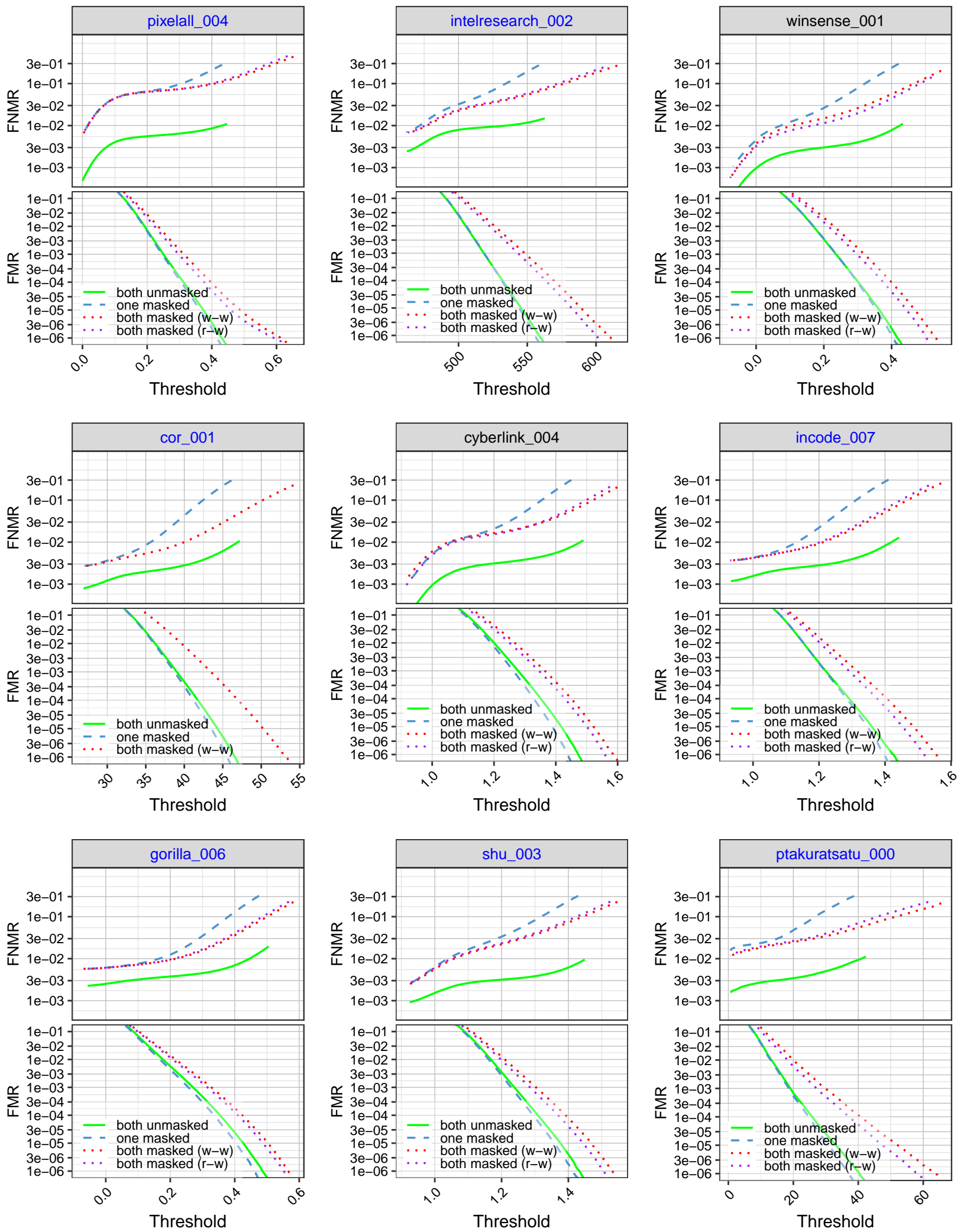

Figure 41: FNMR and FMR calibration curves on unmasked-to-unmasked (both unmasked), masked-to-unmasked with medium, wide, lightblue masks (one masked), masked-to-masked with medium, wide, lightblue masks (both masked (w-w)), and masked-tomasked with medium, round, white masks (enrollment image) and medium, wide, lightblue masks (verification image) (both masked $(r-w))$. Algorithms in black were submitted prior to mid-March 2020, and algorithms in blue were submitted thereafter. 

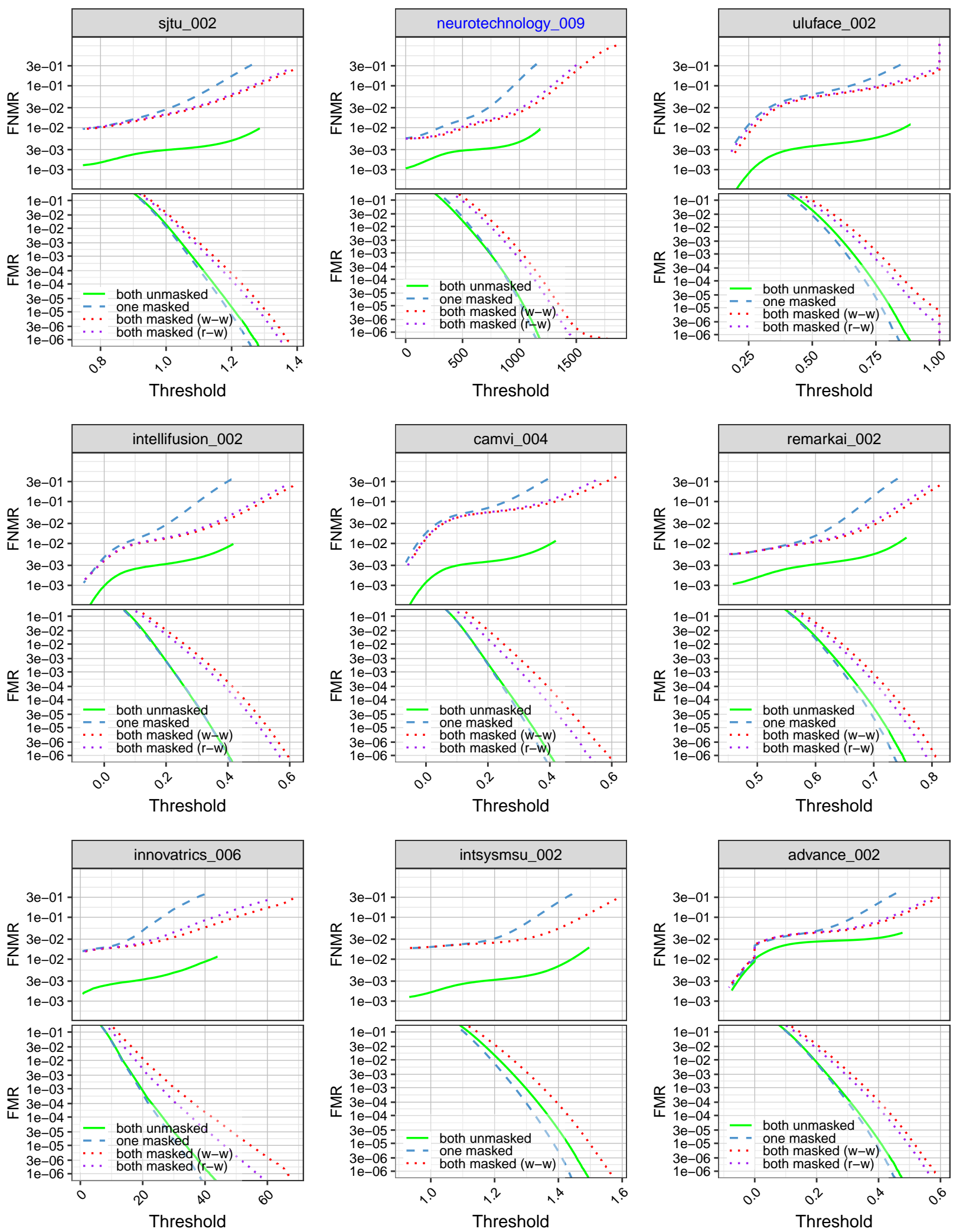

Figure 42: FNMR and FMR calibration curves on unmasked-to-unmasked (both unmasked), masked-to-unmasked with medium, wide, lightblue masks (one masked), masked-to-masked with medium, wide, lightblue masks (both masked (w-w)), and masked-tomasked with medium, round, white masks (enrollment image) and medium, wide, lightblue masks (verification image) (both masked $(r-w))$. Algorithms in black were submitted prior to mid-March 2020, and algorithms in blue were submitted thereafter. 

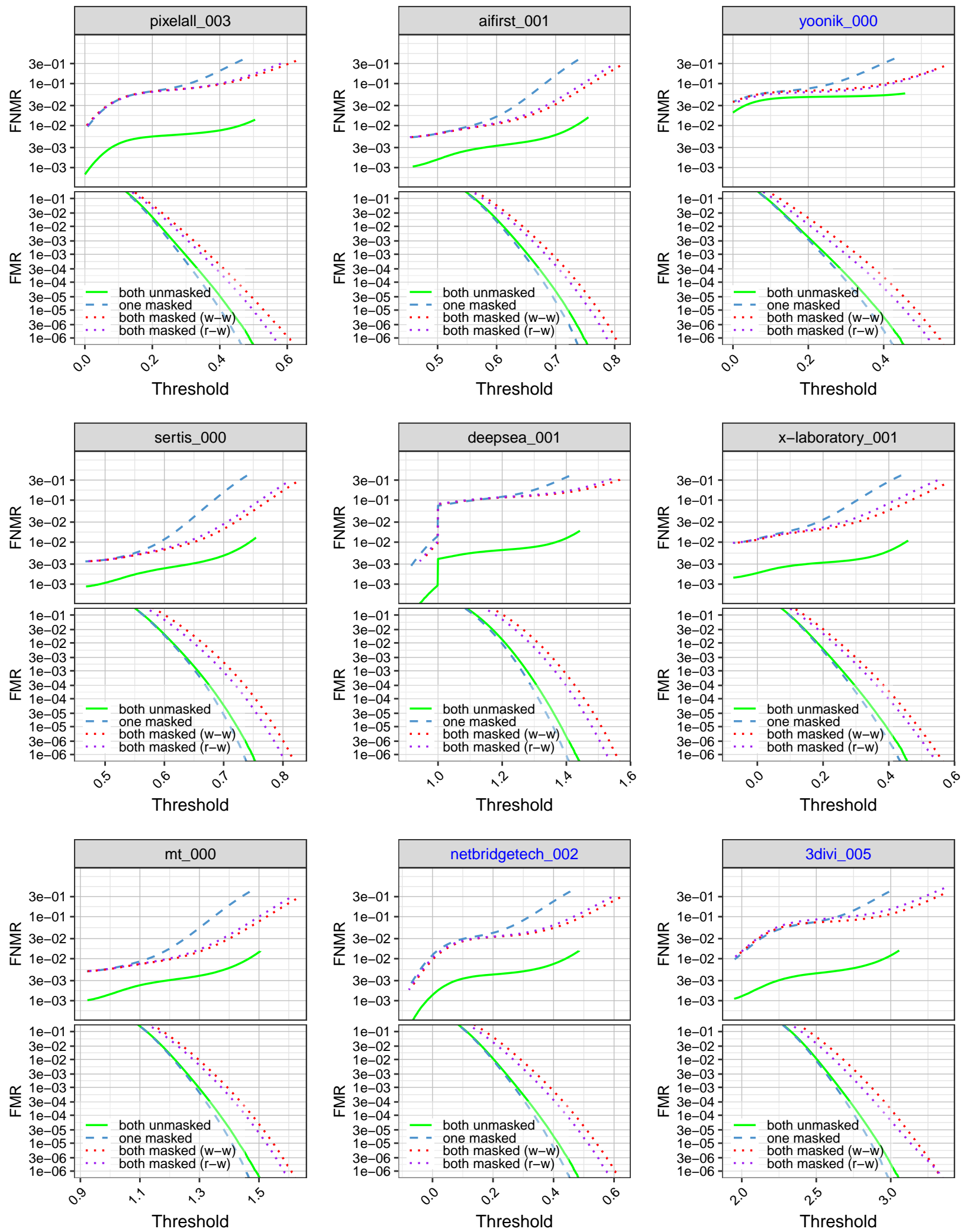

Figure 43: FNMR and FMR calibration curves on unmasked-to-unmasked (both unmasked), masked-to-unmasked with medium, wide, lightblue masks (one masked), masked-to-masked with medium, wide, lightblue masks (both masked (w-w)), and masked-tomasked with medium, round, white masks (enrollment image) and medium, wide, lightblue masks (verification image) (both masked $(r-w))$. Algorithms in black were submitted prior to mid-March 2020, and algorithms in blue were submitted thereafter. 

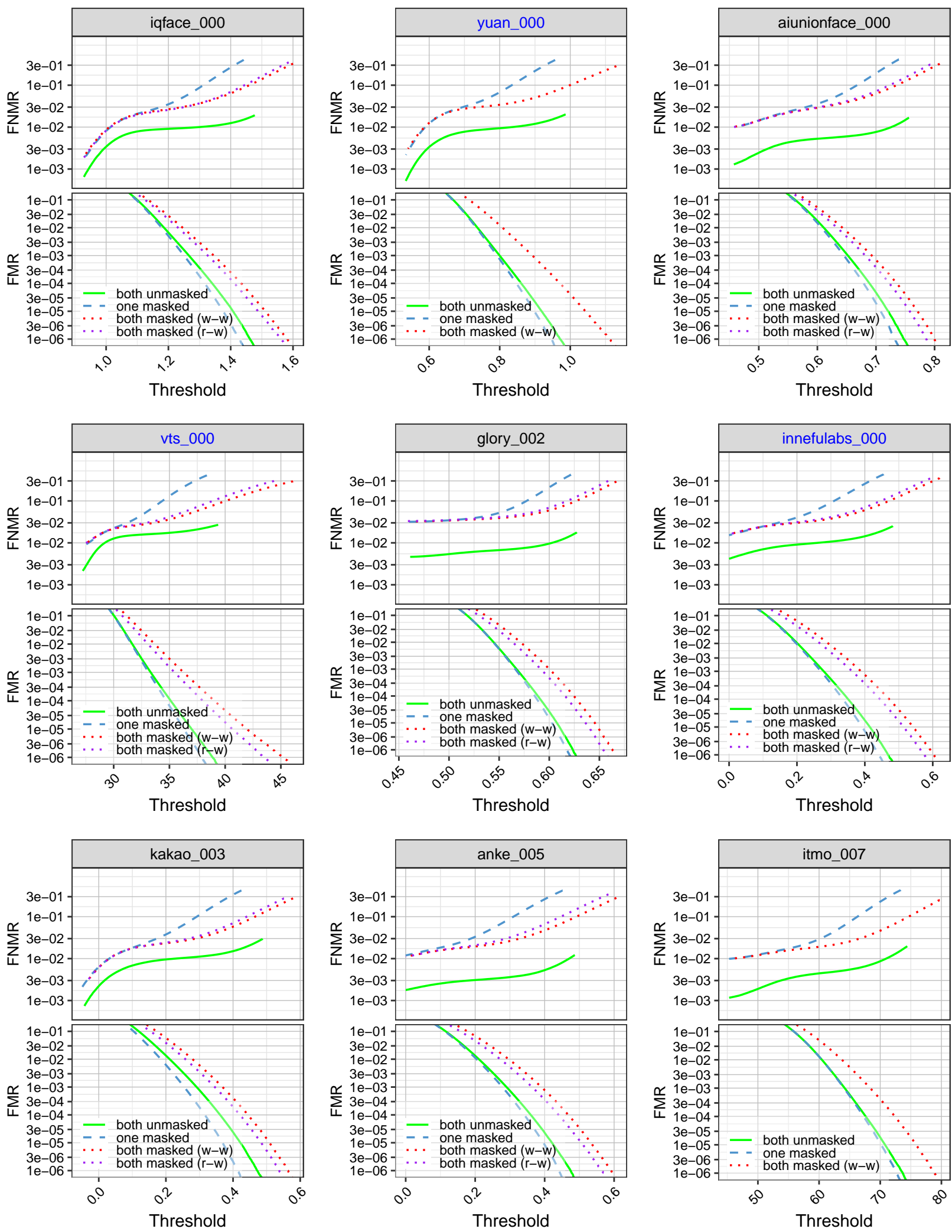

Figure 44: FNMR and FMR calibration curves on unmasked-to-unmasked (both unmasked), masked-to-unmasked with medium, wide, lightblue masks (one masked), masked-to-masked with medium, wide, lightblue masks (both masked (w-w)), and masked-tomasked with medium, round, white masks (enrollment image) and medium, wide, lightblue masks (verification image) (both masked $(r-w))$. Algorithms in black were submitted prior to mid-March 2020, and algorithms in blue were submitted thereafter. 

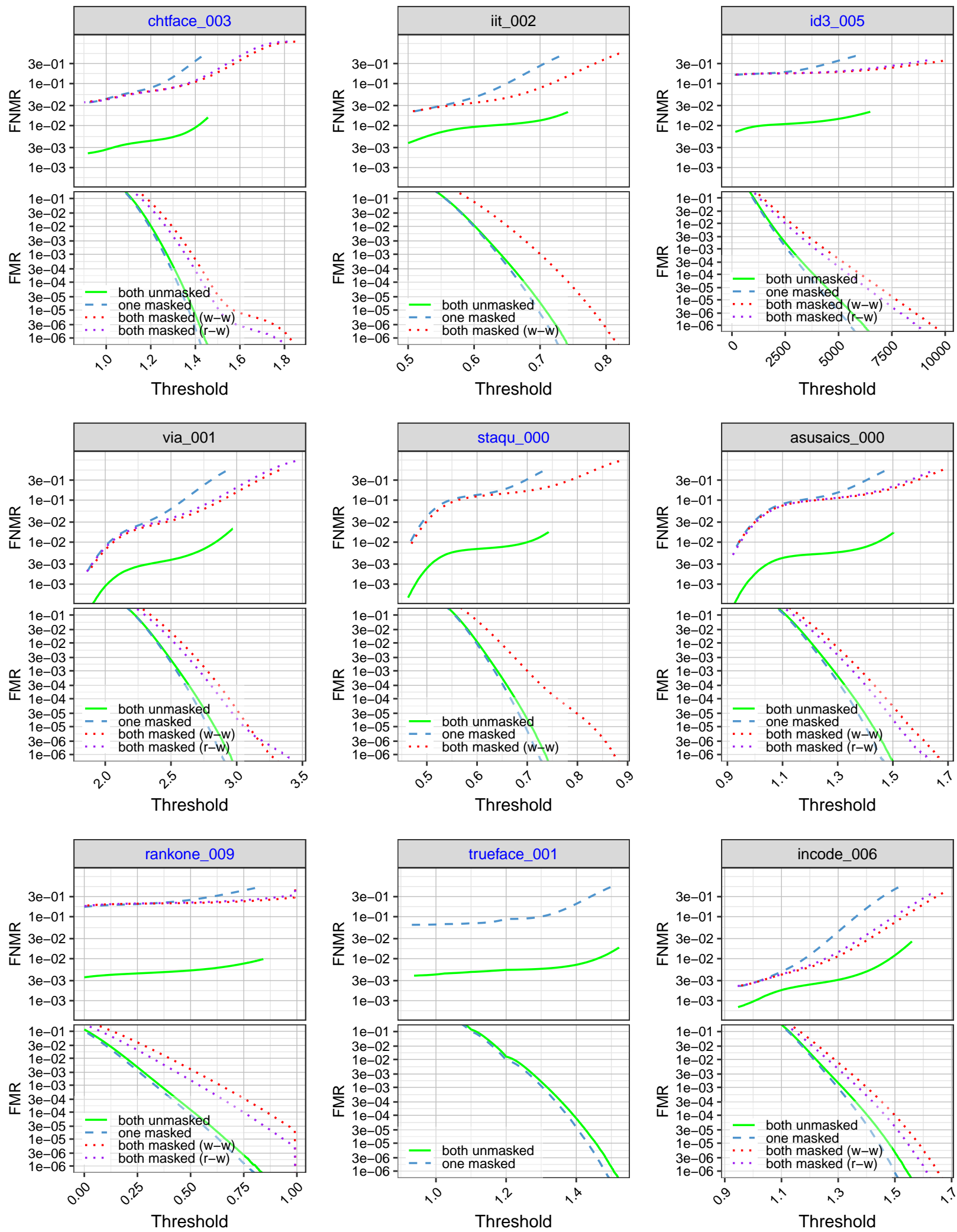

Figure 45: FNMR and FMR calibration curves on unmasked-to-unmasked (both unmasked), masked-to-unmasked with medium, wide, lightblue masks (one masked), masked-to-masked with medium, wide, lightblue masks (both masked (w-w)), and masked-tomasked with medium, round, white masks (enrollment image) and medium, wide, lightblue masks (verification image) (both masked $(r-w))$. Algorithms in black were submitted prior to mid-March 2020, and algorithms in blue were submitted thereafter. 

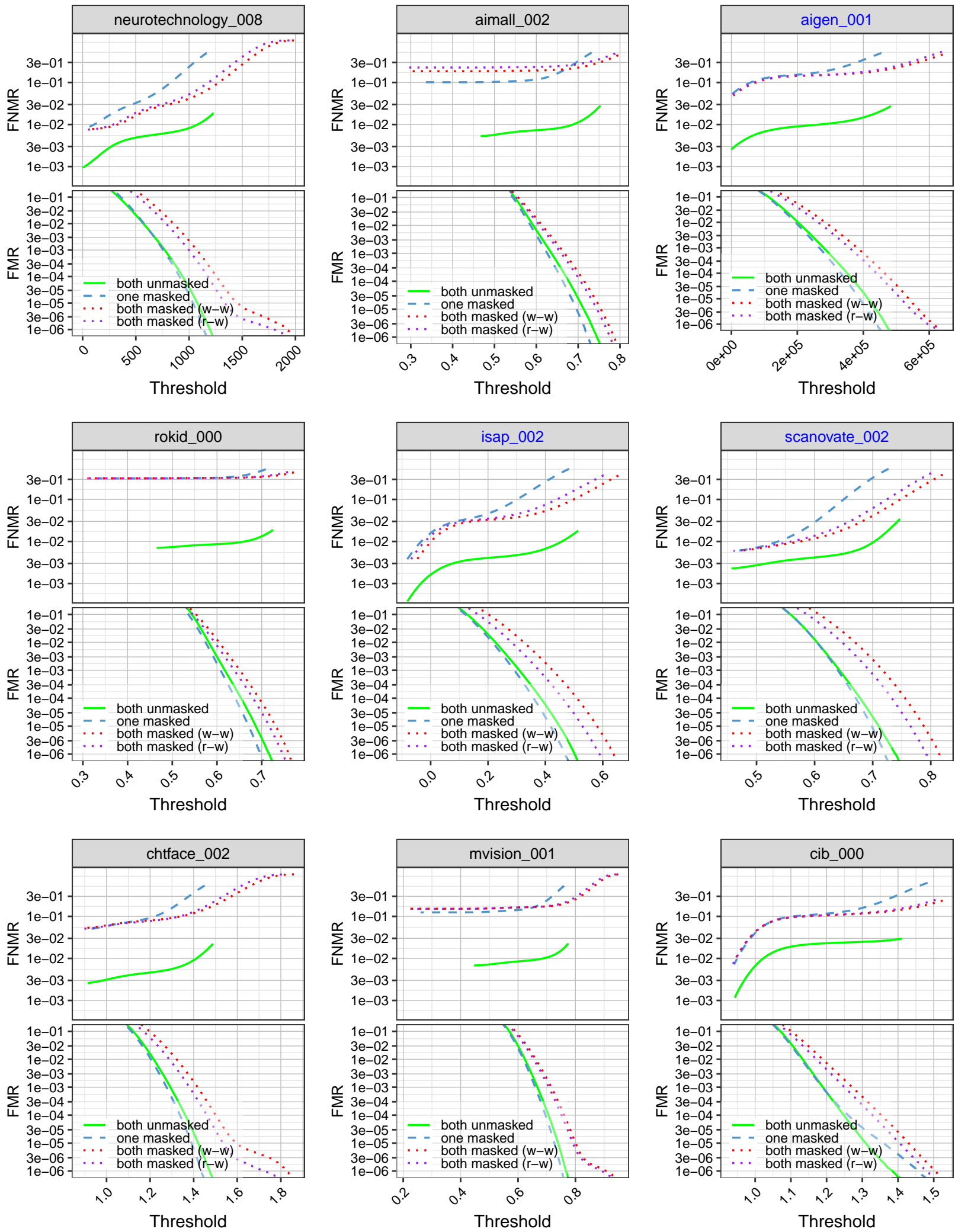

Figure 46: FNMR and FMR calibration curves on unmasked-to-unmasked (both unmasked), masked-to-unmasked with medium, wide, lightblue masks (one masked), masked-to-masked with medium, wide, lightblue masks (both masked (w-w)), and masked-tomasked with medium, round, white masks (enrollment image) and medium, wide, lightblue masks (verification image) (both masked $(r-w))$. Algorithms in black were submitted prior to mid-March 2020, and algorithms in blue were submitted thereafter. 

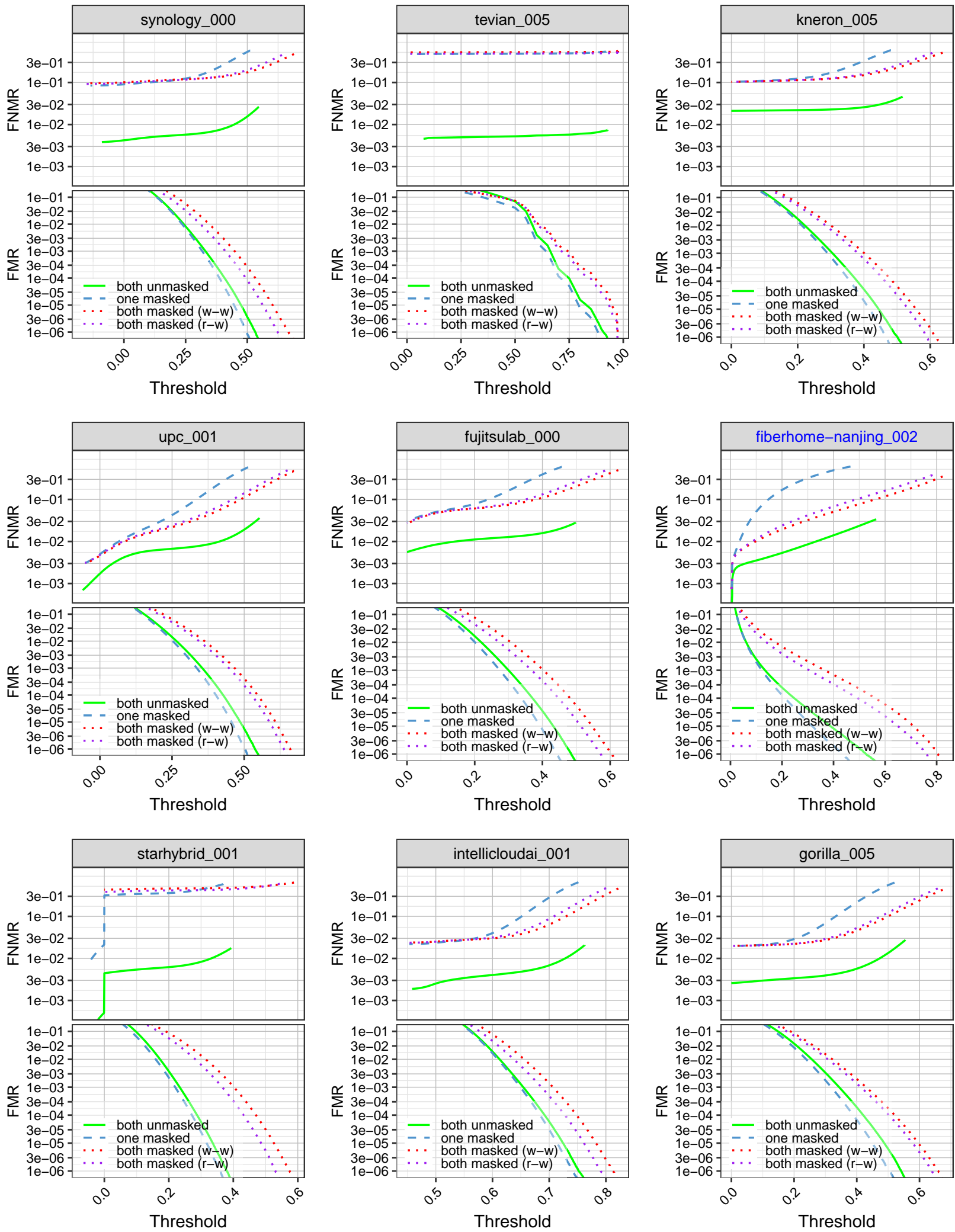

Figure 47: FNMR and FMR calibration curves on unmasked-to-unmasked (both unmasked), masked-to-unmasked with medium, wide, lightblue masks (one masked), masked-to-masked with medium, wide, lightblue masks (both masked (w-w)), and masked-tomasked with medium, round, white masks (enrollment image) and medium, wide, lightblue masks (verification image) (both masked $(r-w))$. Algorithms in black were submitted prior to mid-March 2020, and algorithms in blue were submitted thereafter. 

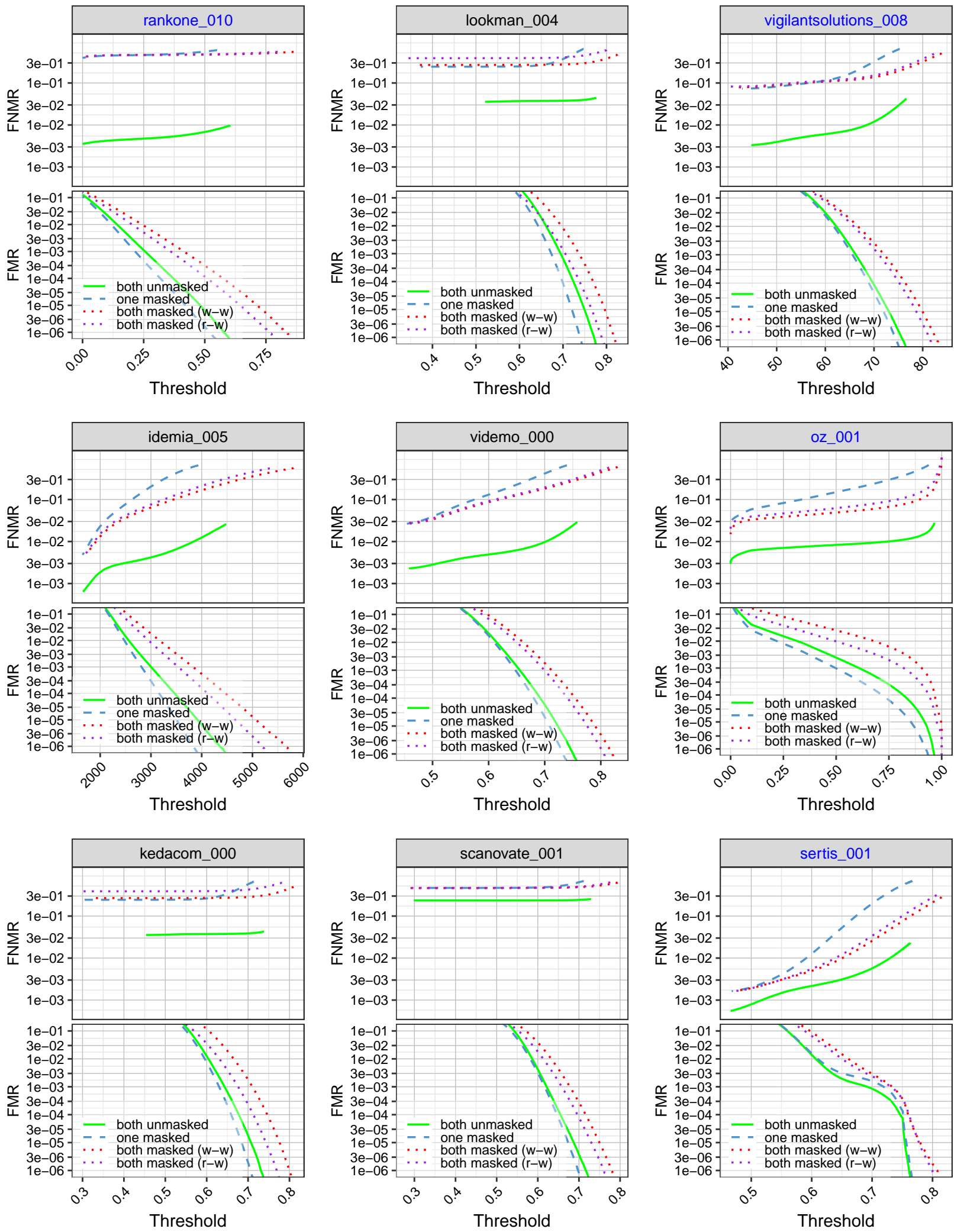

Figure 48: FNMR and FMR calibration curves on unmasked-to-unmasked (both unmasked), masked-to-unmasked with medium, wide, lightblue masks (one masked), masked-to-masked with medium, wide, lightblue masks (both masked (w-w)), and masked-tomasked with medium, round, white masks (enrollment image) and medium, wide, lightblue masks (verification image) (both masked $(r-w))$. Algorithms in black were submitted prior to mid-March 2020, and algorithms in blue were submitted thereafter. 

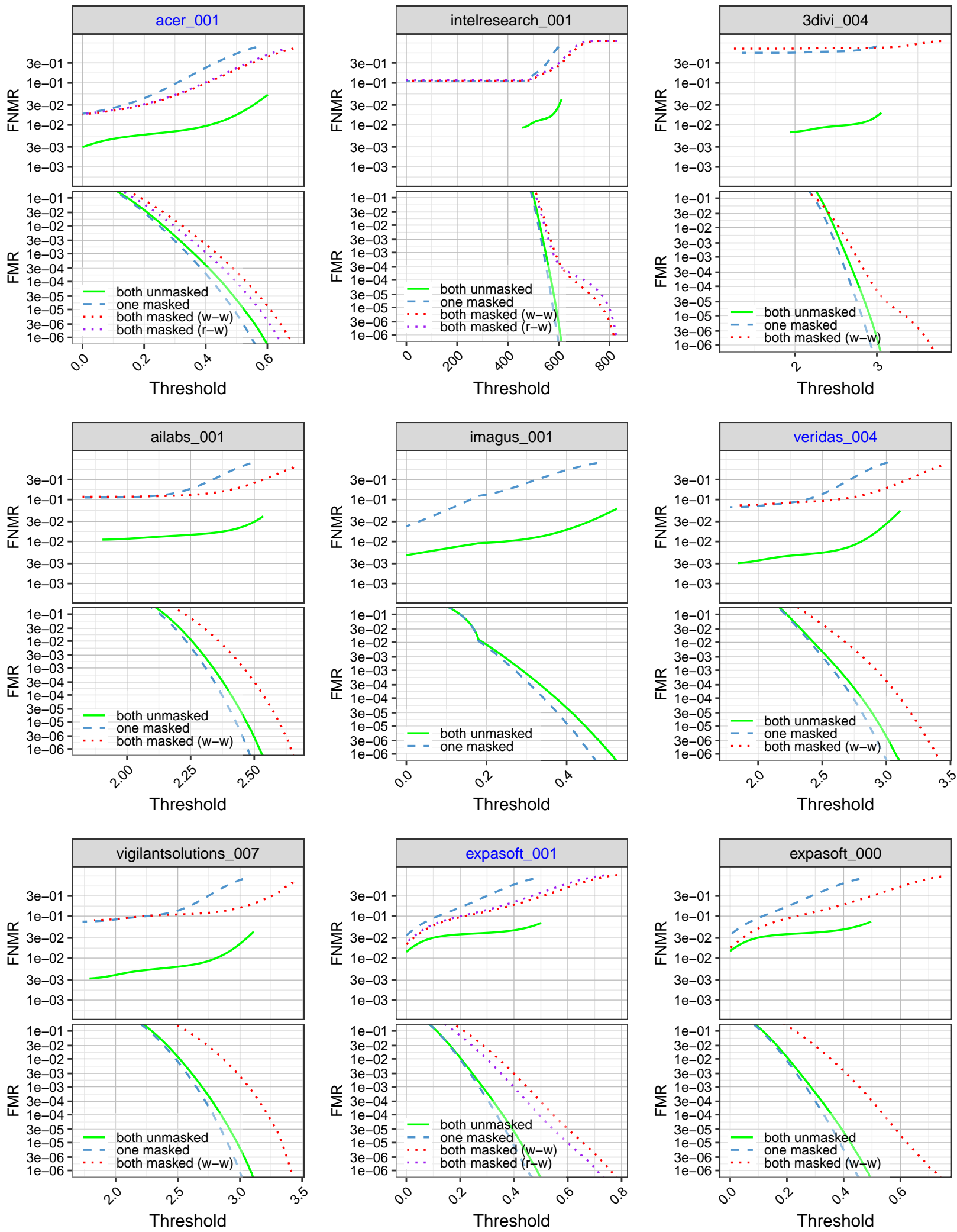

Figure 49: FNMR and FMR calibration curves on unmasked-to-unmasked (both unmasked), masked-to-unmasked with medium, wide, lightblue masks (one masked), masked-to-masked with medium, wide, lightblue masks (both masked (w-w)), and masked-tomasked with medium, round, white masks (enrollment image) and medium, wide, lightblue masks (verification image) (both masked $(r-w))$. Algorithms in black were submitted prior to mid-March 2020, and algorithms in blue were submitted thereafter. 

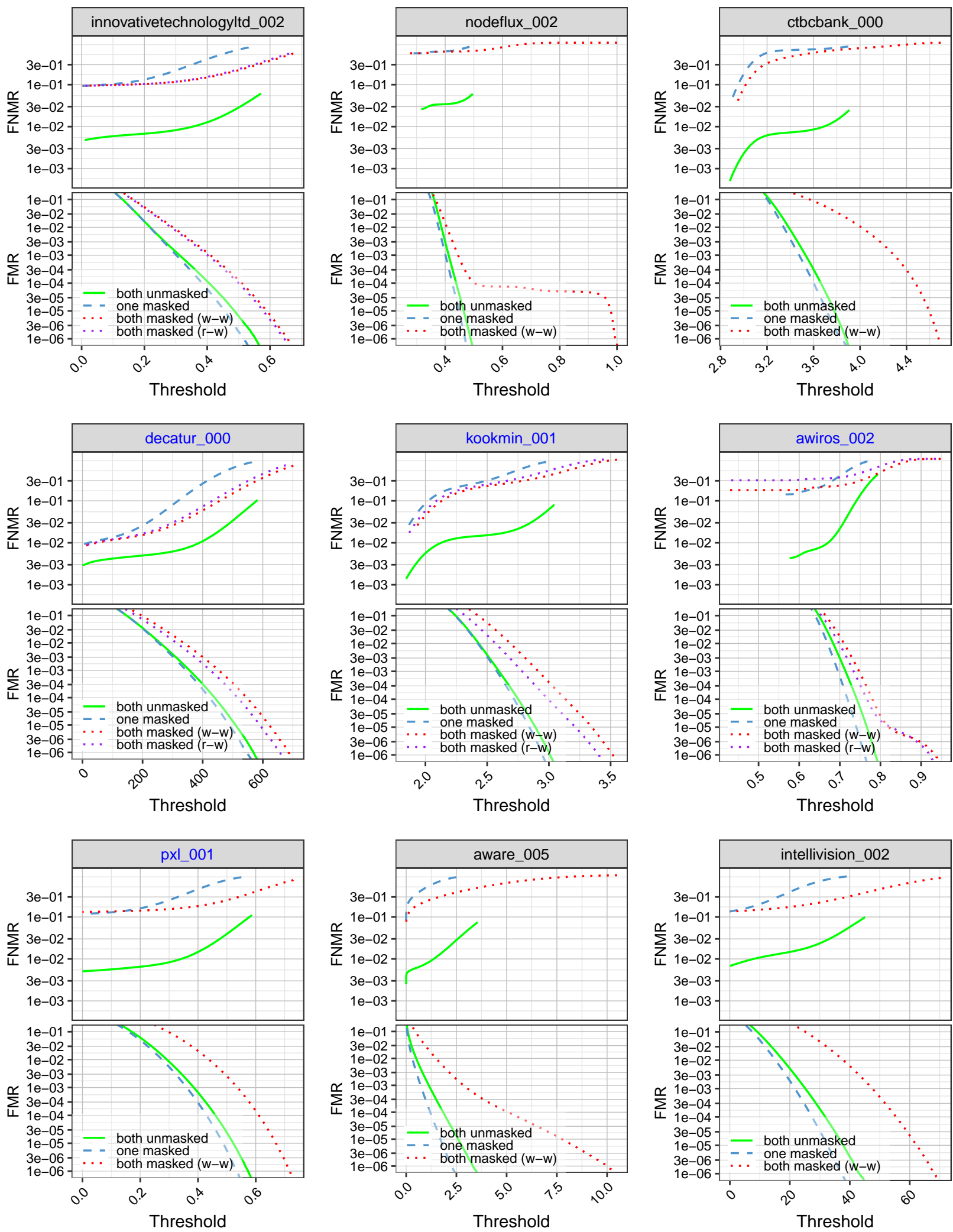

Figure 50: FNMR and FMR calibration curves on unmasked-to-unmasked (both unmasked), masked-to-unmasked with medium, wide, lightblue masks (one masked), masked-to-masked with medium, wide, lightblue masks (both masked (w-w)), and masked-tomasked with medium, round, white masks (enrollment image) and medium, wide, lightblue masks (verification image) (both masked $(r-w))$. Algorithms in black were submitted prior to mid-March 2020, and algorithms in blue were submitted thereafter. 

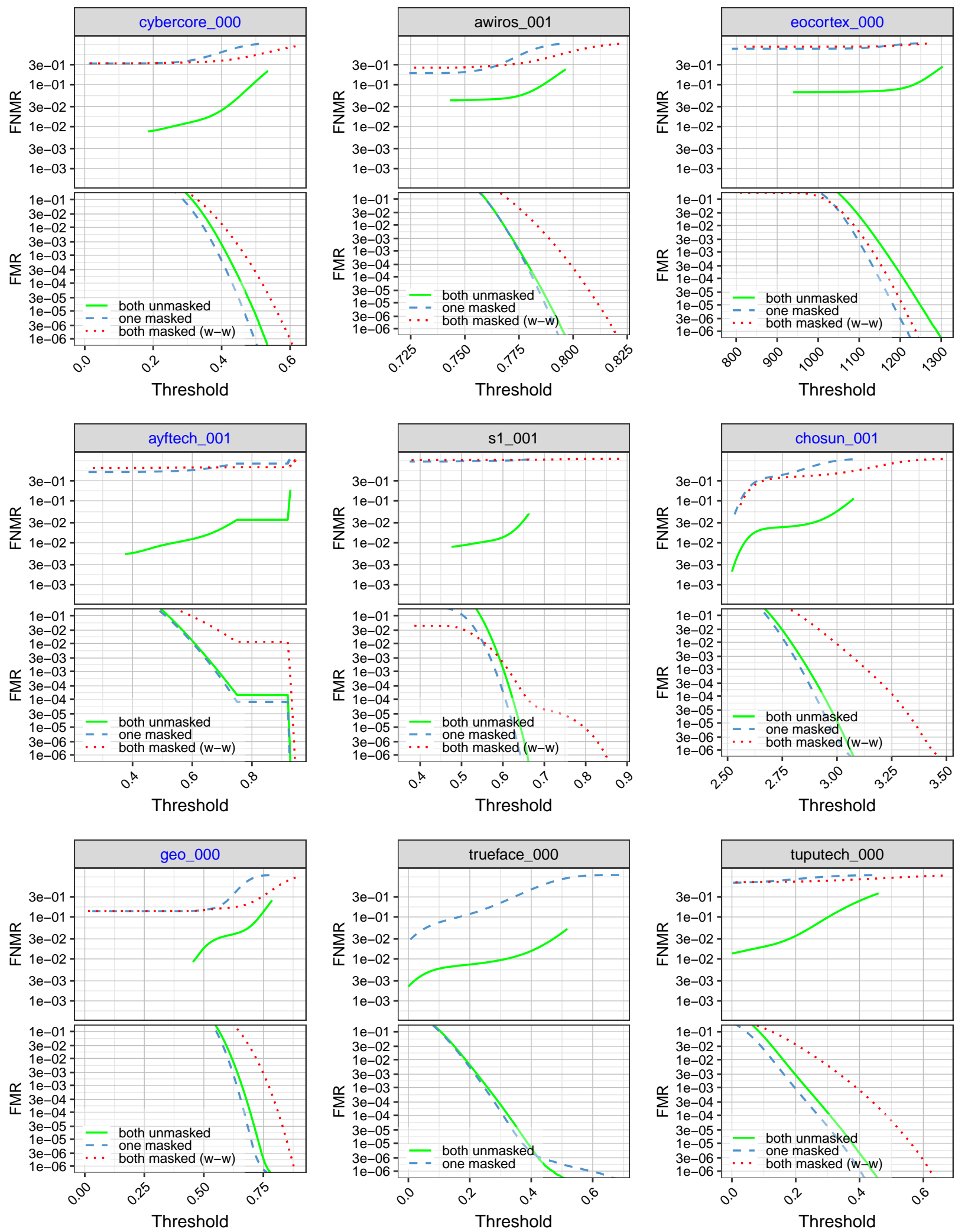

Figure 51: FNMR and FMR calibration curves on unmasked-to-unmasked (both unmasked), masked-to-unmasked with medium, wide, lightblue masks (one masked), masked-to-masked with medium, wide, lightblue masks (both masked (w-w)), and masked-tomasked with medium, round, white masks (enrollment image) and medium, wide, lightblue masks (verification image) (both masked $(r-w))$. Algorithms in black were submitted prior to mid-March 2020, and algorithms in blue were submitted thereafter. 

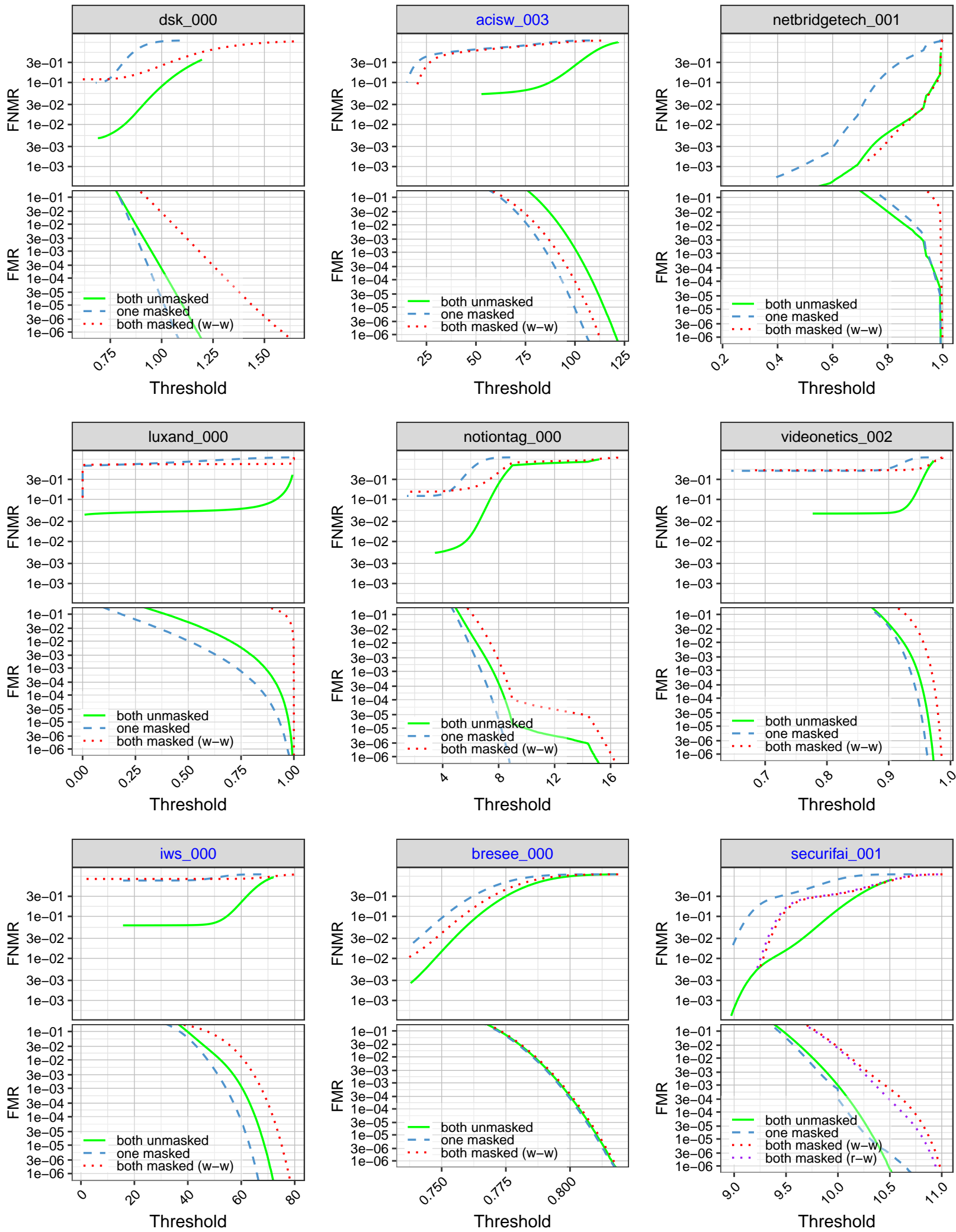

Figure 52: FNMR and FMR calibration curves on unmasked-to-unmasked (both unmasked), masked-to-unmasked with medium, wide, lightblue masks (one masked), masked-to-masked with medium, wide, lightblue masks (both masked (w-w)), and masked-tomasked with medium, round, white masks (enrollment image) and medium, wide, lightblue masks (verification image) (both masked $(r-w))$. Algorithms in black were submitted prior to mid-March 2020, and algorithms in blue were submitted thereafter. 

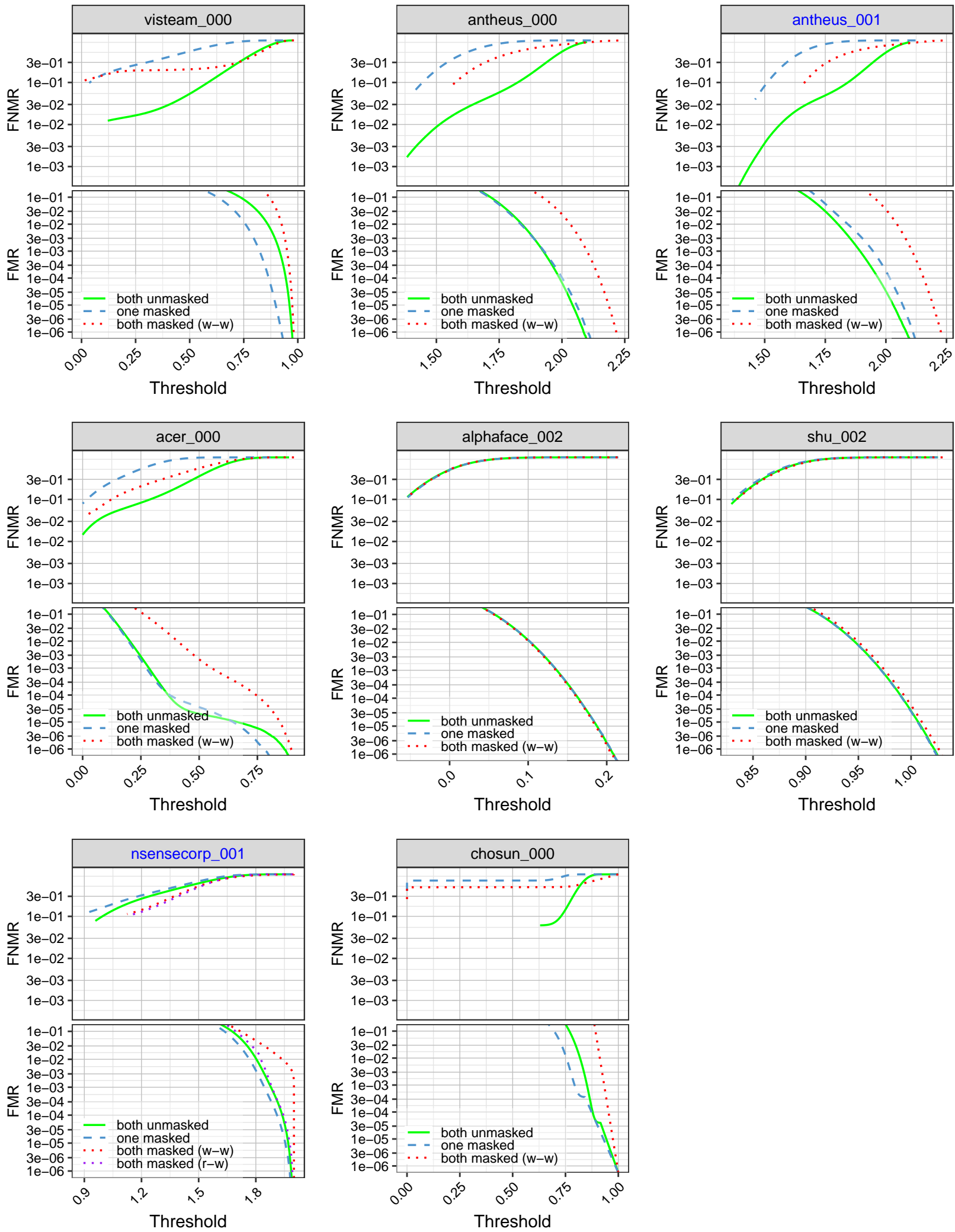

Figure 53: FNMR and FMR calibration curves on unmasked-to-unmasked (both unmasked), masked-to-unmasked with medium, wide, lightblue masks (one masked), masked-to-masked with medium, wide, lightblue masks (both masked (w-w)), and masked-tomasked with medium, round, white masks (enrollment image) and medium, wide, lightblue masks (verification image) (both masked $(r-w))$. Algorithms in black were submitted prior to mid-March 2020, and algorithms in blue were submitted thereafter. 


\section{References}

[1] ISO/IEC 19794-5:2011 - Information technology - Biometric data interchange formats - Part 5: Face image data.

[2] N95 Respirators, Surgical Masks, and Face Masks. https://www.fda. gov/medical-devices/personal-protective-equipment-infection-control/ n95-respirators-surgical-masks-and-face-masks.

[3] NIST Special Database 32 - Multiple Encounter Dataset (MEDS-II). https://www.nist.gov/itl/iad/ image-group/special-database-32-multiple-encounter-dataset-meds.

[4] Patrick Grother, Mei Ngan, and Kayee Hanaoka. NISTIR 8311 - Ongoing FRVT Part 6A: Face recognition accuracy with face masks using pre-COVID-19 algorithms. Interagency Report 8311, National Institute of Standards and Technology, July 2017. https:/ / pages.nist.gov/frvt/reports/11/frvt_11_report_2017_07_29.pdf.

[5] Patrick Grother, George Quinn, and Mei Ngan. Face in video evaluation (five) face recognition of noncooperative subjects. Interagency Report 8173, National Institute of Standards and Technology, March 2017. https://doi.org/10.6028/NIST.IR.8173.

[6] J. Hanley and A. Lippman-Hand. If nothing goes wrong, is everything all right? interpreting zero numerators. JAMA, 249 13:1743-5, 1983.

[7] Davis E. King. Dlib-ml: A machine learning toolkit. Journal of Machine Learning Research, 10:1755-1758, 2009. http: //dlib.net.

[8] Mei Ngan, Patrick Grother, and Kayee Hanaoka. NISTIR 8311 - Ongoing FRVT Part 6A: Face recognition accuracy with face masks using pre-COVID-19 algorithms. Interagency Report 8311, National Institute of Standards and Technology, July 2020. https:/ / doi.org/10.6028/NIST.IR.8311.

[9] Patrick Grother and Mei Ngan and Kayee Hanaoka. NIST Ongoing Face Recognition Vendor Test (FRVT) 1:1 Verification Application Programming Interface (API), April 2019. https://pages.nist.gov/frvt/api/FRVT_ ongoing_11_api_4.0.pdf.

[10] Zhongyuan Wang, Guangcheng Wang, Baojin Huang, Zhangyang Xiong, Qi Hong, Hao Wu, Peng Yi, Kui Jiang, Nanxi Wang, Yingjiao Pei, Heling Chen, Yu Miao, Zhibing Huang, and Jinbi Liang. Masked face recognition dataset and application, 2020. 


\section{Appendix A Dlib Masking Methodology}

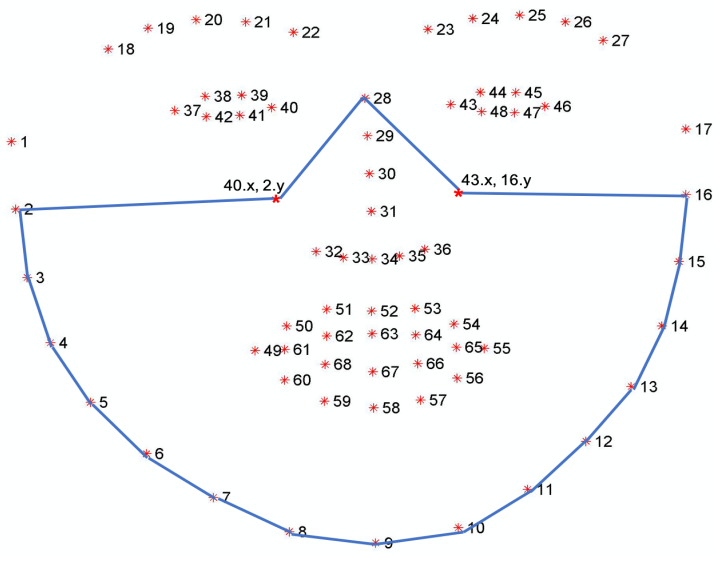

(a) wide, high coverage

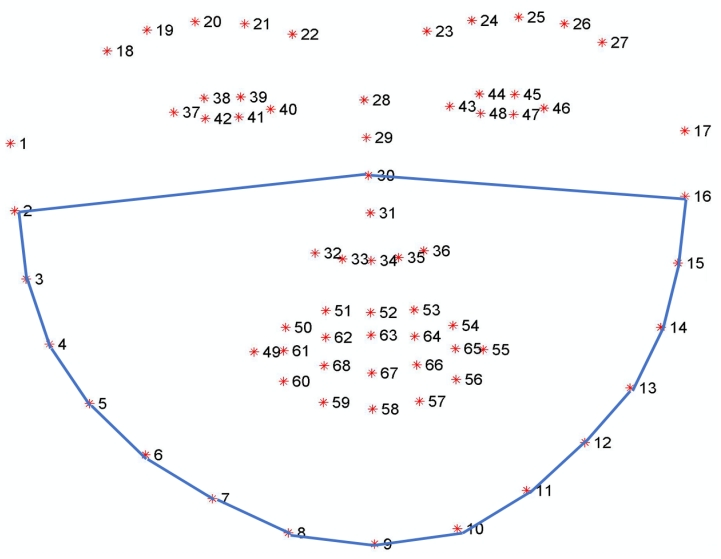

(c) wide, medium coverage

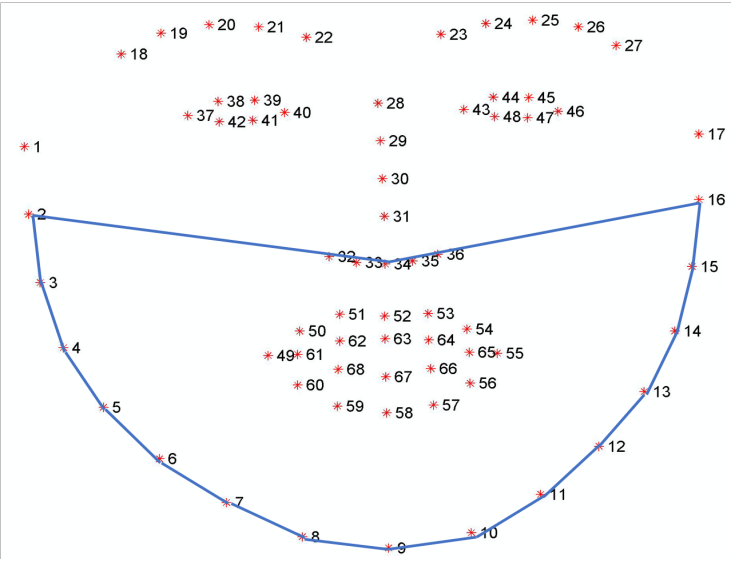

(e) wide, low coverage

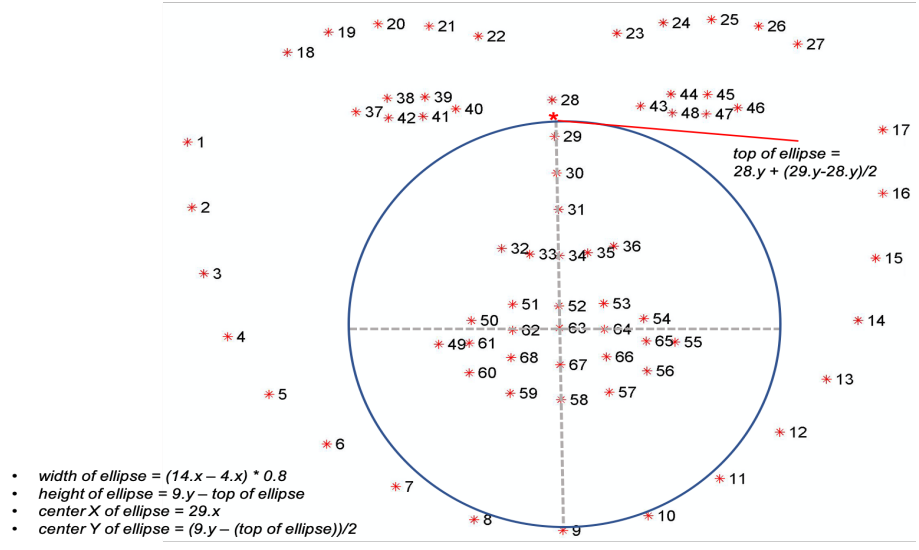

(b) round, high coverage

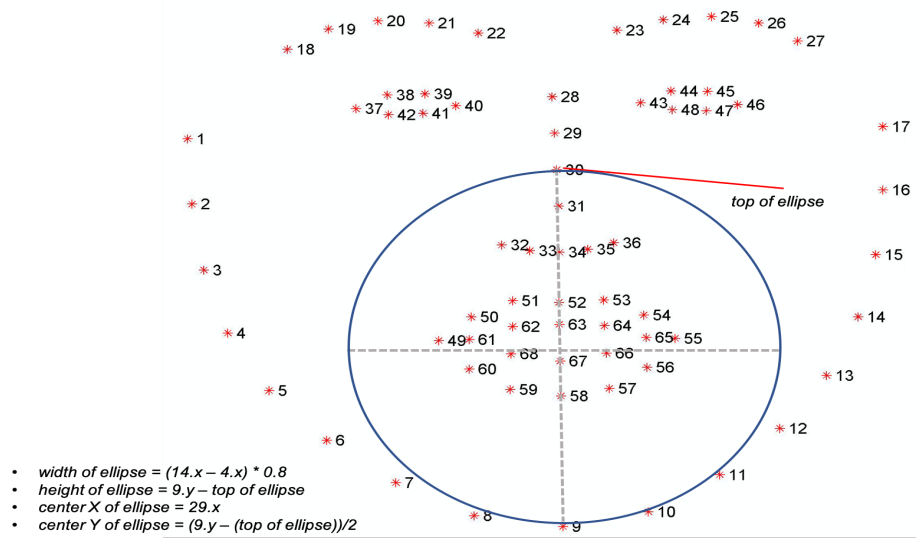

(d) round, medium coverage

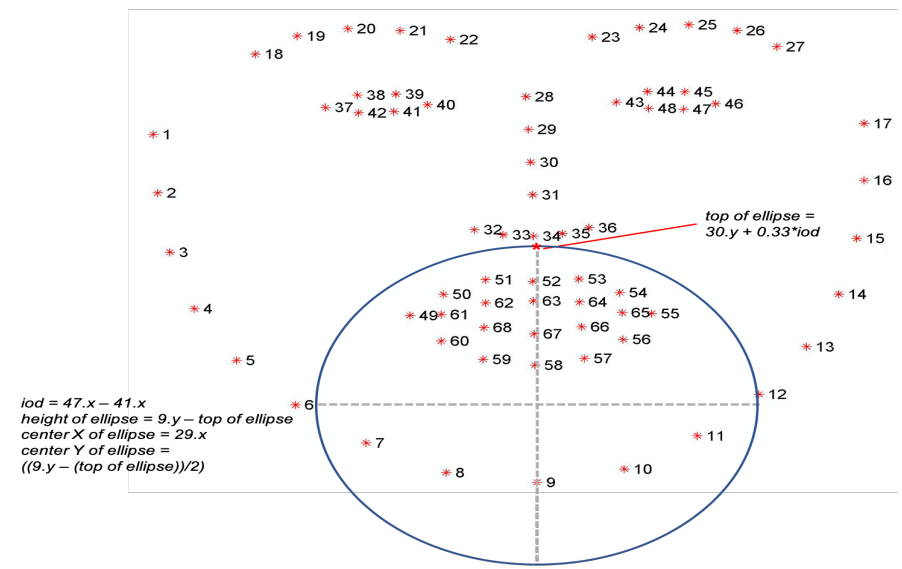

(f) round, low coverage

Figure 54: This figure shows the Dlib facial points used to create the various synthetic masks used in this report. For wide masks, the specified Dlib facial points were used to generate a closed polygon and two additional points were interpolated between each dlib facial point used for smoothing purposes. For round masks, the specified Dlib facial points were used to generate an ellipse. The Dlib $\mathrm{C}++$ toolkit version 19.19, configured with the common histogram of gradients (HoG)-based face detector and 68 face landmark shape predictor was used to generate the 68 facial landmarks. 